\title{
Revision of the genus Pseudognaphalium (Asteraceae, Gnaphalieae) in Chile
}

\author{
Revisión del género Pseudognaphalium (Asteraceae, Gnaphalieae) en Chile
}

\author{
Susana E. Freire ${ }^{1,2 *}$, Néstor D. Bayón², Carlos M. Baeza³ ${ }^{3}$ Daniel A. Giuliano² \& Claudia Monti² \\ ${ }^{1}$ Instituto de Botánica Darwinion, Labardén 200, CC 22, B1642HYD San Isidro, Buenos Aires, Argentina. \\ 2Área de Botánica, Departamento de Ciencias Biológicas, Facultad de Ciencias Agrarias y Forestales, Universidad Nacional \\ de La Plata, Avda. 60 entre 116 y 118, 1900 La Plata, Argentina. \\ ${ }^{3}$ Facultad de Ciencias Naturales y Oceanográficas, Departamento de Botánica, Casilla 160-C, Universidad de Concepción, \\ Concepción, Chile. \\ *sfreire@darwin.edu.ar
}

\begin{abstract}
The genus Pseudognaphalium is one of the largest genera of the tribe Gnaphalieae (Asteraceae) with about 90 species with worldwide distribution. The vast majority of species are found in South, Central and North America, but some species also occur in Asia and Africa. This work includes the revision of 12 species of Pseudognaphalium from Chile, P. aldunateoides, P. cabrerae, P. cheiranthifolium, P. cymatoides, P. gayanum, P. lacteum, P. landbeckii, P. munoziae, P. psilophyllum, P. remyanum, $P$. tarapacanum, and $P$. viravira. A key is provided for their identification, as well as morphological description, illustration, geographic distribution and habitat, distribution maps, phenology, additional material examined, distinguishing characters and taxonomic affinities for each taxon. The new combination Pseudognaphalium cabrerae (S.E. Freire) S.E. Freire, N. Bayón, C. Baeza, Giuliano \& C. Monti, is proposed. The following taxa are newly synonymized: Gnaphalium diminutivum, Pseudognaphalium perpusillum, with P. aldunateoides; G. canum, G. ulophyllum, P. moelleri with $P$. cymatoides; $P$. heterophyllum, $P$. heterotrichum, G. pseudohelichrysum, P. robustum with $P$. gayanum; P. glandulosum with P. psilophyllum; G. longifolium, G. subnudum, P. andicola, P. coquimbense, P. illapelinum, P. montevidense, P. pratense, with $P$. viravira. Lectotypes are newly designated for Gnaphalium acutifolium, G. araucanum, G. argyrolepis, G. canum, G. cymatoides, G. glandulosum, G. heterophyllum, G. illapelinum, G. lacteum, G. landbeckii, G. moelleri, G. ramosum, G. remyanum, G. subnudum, G. tarapacanum, G. ulophyllum, and G. viravira. A neotype is designated for G. psilophyllum.
\end{abstract}

KeYwords: Gnaphalium, lectotypification, neotype, South America, synonyms, taxonomy.

\section{RESUMEN}

El género Pseudognaphalium, con cerca de 90 especies de distribución cosmopolita, es uno de los géneros de la tribu Gnaphalieae con mayor número de especies, principalmente distribuidas en América del Sur, Central y del Norte, aunque algunas también están presentes en Asia y África. Este trabajo comprende la revisión de 12 especies de Pseudognaphalium para Chile, $P$. aldunateoides, $P$. cabrerae, P. cheiranthifolium, P. cymatoides, $P$. gayanum, P. lacteum, P. landbeckii, $P$. munoziae, $P$. psilophyllum, $P$. remyanum, $P$. tarapacanum y $P$. viravira. Se incluye una clave para identificarlas y para cada una de las especies se brinda descripción, ilustración, fenología, distribución geográfica y hábitat, mapa de distribución, material adicional examinado y afinidades taxonómicas. Sobre la base del estudio de los materiales tipo, se establecen los siguientes nuevos sinónimos: Gnaphalium diminutivum, Pseudognaphalium perpusillum, con P. aldunateoides; G. canum, G. ulophyllum, P. moelleri con P. cymatoides; P. heterophyllum, P. heterotrichum, G. pseudohelichrysum, P. robustum, con P. gayanum; P. glandulosum con P. psilophyllum; G. longifolium, G. subnudum, P. andicola, P. coquimbense, $P$. illapelinum, $P$. montevidense, $P$. pratense, con P. viravira; se designan lectotipos para las siguientes especies: Gnaphalium acutifolium, G. araucanum, G. argyrolepis, G. canum, G. cymatoides, G. glandulosum, G. heterophyllum, G. illapelinum, G. lacteum, G. landbeckii, G. moelleri, G. ramosum, G. remyanum, G. subnudum, G. tarapacanum, G. ulophyllum, G. viravira y se designa el neotipo para G. psilophyllum.

Palabras clave: Gnaphalium, lectotipificación, neotipo, sinónimos, Sud América, taxonomía. 


\section{INTRODUCTION}

The cosmopolitan genus Pseudognaphalium Kirp. was based on a Mexican species, Gnaphalium oxyphyllum DC. It is one of the most species-rich genera of the tribe Gnaphalieae (Asteraceae) with about 90 species of dwarf prostrate to robust erect herbs. The vast majority of species are distributed in South, Central and North America, but some species also occur in Asia and Africa (Hilliard \& Burtt 1981, Anderberg 1991, Bayer et al. 2007).

Pseudognaphalium was treated in regional works or floral treatments, such as Rémy (1849, as Gnaphalium L.) and Reiche (1905, as Gnaphalium) for Chile; Cabrera (1963, 1971, 1974, 1978, as Gnaphalium) for Argentina; Hilliard (1983) and Hilliard \& Burtt (1981) for South Africa; Dillon \& Sagástegui-Alva (1991a, b, as Gnaphalium) for Peru; Freire (1998, as Gnaphalium) for Paraguay; Nesom (2001, 2004, 2006) and Ballard et al. ( 2004) for North America; Deble \& Marchiori (2006), and Freire et al. (2011) for Brazil; Hind (2011) for Bolivia, and Chen et al. (2011) for Asia.

Cladistic analysis of morphological characters (Anderberg 1991) and recent results from molecular studies (e.g. Ward et al. 2009) have been interpreted as providing support for recognizing Pseudognaphalium as a distinct genus from Gnaphalium. The genus Pseudognaphalium includes taxa with monochromous phyllaries with divided stereomes, monomorphic pappus bristles, clusters of capitula arranged in corymbs or panicles, and the achenes either glabrous or with short oblong myxogenic duplex hairs. In contrast, Gnaphalium includes taxa with polychromous phyllaries with entire stereomes, dimorphic pappus bristles, few capitula in sometimes leafy clusters, and pilose achenes with oblong non-myxogenic duplex hairs (Drury 1970, under 'achyroclinoid' cudweeds; Hilliard 1983, Hilliard $\&$ Burtt 1981, Anderberg 1991). Furthermore, these and other more recent molecular studies (Smissen et al. 2011, Nie et al. 2013) noted that Pseudognaphalium is more closely related to Helichrysum Mill. and Anaphalis DC. than to Gnaphalium. Morphologically Pseudognaphalium, including Laphangium (Hilliard \& B.L. Burtt) Tzvelev, resembles the genera Helichrysum and Anaphalis in having divided stereome, receptacle smooth or honeycombed, achenes glabrous or short pilose, and pappus uniseriate with barbellate setae (Hilliard \& Burtt 1981). It differs from Helichrysum, however, in having pistillate florets always outnumbering the bisexual ones (vs. pistillate florets not outnumbering the bisexual florets), and from Anaphalis, in having bisexual central florets (vs. functionally male).

Since the first taxonomic treatments of Chilean species of Pseudognaphalium (under Gnaphalium, including Gamochaeta Wedd.) were carried out by Rémy (1849), Philippi (1895), and Reiche (1905), new species were described or redescribed without a new broad taxonomic evaluation or revision. Catalogues concerning aspects of nomenclature of Pseudognaphalium (under Gnaphalium, excluding Gamochaeta) by Marticorena \& Quezada (1985), and Freire \& Iharlegui (2008) clarified synonyms and superfluous names. Nevertheless, the revision of species in Chile highlighted the need for further taxonomic adjustments.

The main goal of this study was to revise Chilean species of Pseudognaphalium, considering morphological characters and geographic distribution. The present study also fully describes, illustrates, and provides a key to twelve species occurring in Chile.

\section{MATERIALS AND METHODS}

The study is based on herbarium material from the following herbaria (abbreviations according to Thiers 2011): BAB, CONC, GH, LP, LPAG, MO, NY, PH, SGO, and SI. Electronic images of type specimens at E, G-DC, L, P, SGO, were also studied. Pappus bristles were observed after boiling in water and staining with $2 \%$ safranin. Characteristics of phyllaries and trichomes were observed and recorded in cleared samples using Dizeo de Strittmatter's technique (1973). Observations were carried out using Gemalux (Light Microscopy), equipped with a photographic camera PAL CCD. Trichome terminology follows Ramayya (1962). Leaf descriptions were made utilizing the terminology of Harris \& Harris (1994).

\section{MORPHOLOGY AND MICROMORPHOLOGY}

Habitat and stems. Most Chilean species of Pseudognaphalium are perennial herbs or subshrubs, more rarely biennials or annuals, i.e. P. aldunateoides, $P$. cymatoides, ranging from few $\mathrm{cm}(P$. aldunateoides, P. lacteum) to up to $110 \mathrm{~cm}$ tall (P. gayanum), simple (or $2-3$, e.g. $P$. cabrerae) or more usually with primary stem branching at ground level only, and erect or ascending unbranched stems from a decumbent base.

Pubescence. Pubescence in Chilean species of Peudognaphalium consists of two basic trichome types. The type of pubescence is often useful alone or in combination with other characters in distinguishing species (e.g., $P$. cheiranthifolium from P. cabrerae). Two basic types of hairs, present on stems, leaves, and phyllaries, are described below.

1. Long uniseriate eseptate flagellate trichomes (Fig. 1A). This type of trichome consists of one or two foot cells, two-seven-celled stalks or stipes, and a single very long, flagellate, eseptate apical cell. This trichome type is present in all Chilean species of Pseudognaphalium, and especially in Pseudognaphalium aldunateoides, P. cabrerae, P.lacteum, $P$. landbeckii, $P$. remyanum, and $P$. viravira, where they cover almost the entire surface of the leaf, with few short 
glandular trichomes hidden under the wool.

2. Biseriate glandular trichomes (Fig. 1B, C). Comprised of 2 rows of cells in the body, with three to many cells in each row, enclosed by a persistent or collapsed cuticular vesicle. These trichomes vary from short clavate to long biseriate, $0.05-0.5 \mathrm{~mm}$ long. Glandular hairs are widespread in all the taxa studied, and especially in Pseudognaphalium cymatoides, P. gayanum, P. munoziae, P. psilophyllum, and $P$. tarapacanum, where they cover almost the entire surface of the leaf, with few flagellate trichomes. Only in $P$. cheiranthifolium, long glandular trichomes are raised over woolly trichomes.

Achenial PUbESCEnCE. Short pilose achenes occur in a few Chilean species, i.e., $P$. aldunateoides, $P$. cymatoides, $P$. gayanum (rarely $P$. tarapacanum, $P$. viravira). These are twin hairs or duplex myxogenic hairs and consist of two small, square basal cells and two short apical cells rounded at the apex and equal in length (Fig. 2A, D). The remaining taxa have glabrous achenes with smooth or papillose epidermis (Fig. 2B, C, E).

LEAves. All taxa analyzed have alternate, usually approximated at the base, occasionally rosulate (Pseudognaphalium munoziae), sessile leaves. The leaves are always simple, predominantly linear, linear-lanceolate, lanceolate or obovate. Only two species have conspicuously undulate margins i.e. Pseudognaphalium cymatoides and $P$. gayanum. In the remaining taxa the leaf margin is predominantly flat. The leaf base is often stem-clasping and decurrent.
InfloResCence. The capitula in Chilean Pseudognaphalium are arranged in a cymose pattern (Endress 2010). The first-order axis (branch) has two short second-order axes (branches), and these short lateral branches of each axis have two or three branching orders, resulting a dense terminal cluster (Fig. 3A, P. tarapacanum, P. viravira). However in most species, clusters or cymose units are arranged in thyrsoid inflorescence (Troll 1964-1969, Weberling 1985, Rua 1999, Endress 2010) of three cymose units, commonly called 'corymbs' (Fig. 3B, P. aldunateoides, P. cymatoides, $P$. cheiranthifolium, P. cabrerae, P. gayanum, P. lacteum, $P$. psilophyllum, $P$. viravira) or with more cymose units, commonly called 'panicle' (Fig. 3C, P. aldunateoides, P. cymatoides, P. gayanum, P. lacteum, P. landbeckii, P. munoziae, P. psilophyllum, P. remyanum).

CAPITUla. The capitula of Pseudognaphalium are heterogamous and discoid, containing in Chilean species up to $(51,74)$ 95-212 florets with pistillate flowers usually more than 10 times as many as bisexual flowers. Campanulate involucres are characteristic of most species of this study, ranging from 2 to $6 \mathrm{~mm}$ high.

Phyllaries. The phyllaries are arranged in 3 or 4 imbricate series, with the inner equalling or slightly exceeding the flowers, and not conspicuously longer than the outer. The outer phyllaries are dorsally glandular pubescent and the inner glandular or glabrous. The phyllary lamina is monochromous opaque-white or hyaline, whitish, yellowish or brownish and shiny, and the stereome is divided in all taxa studied (Fig. 4A).
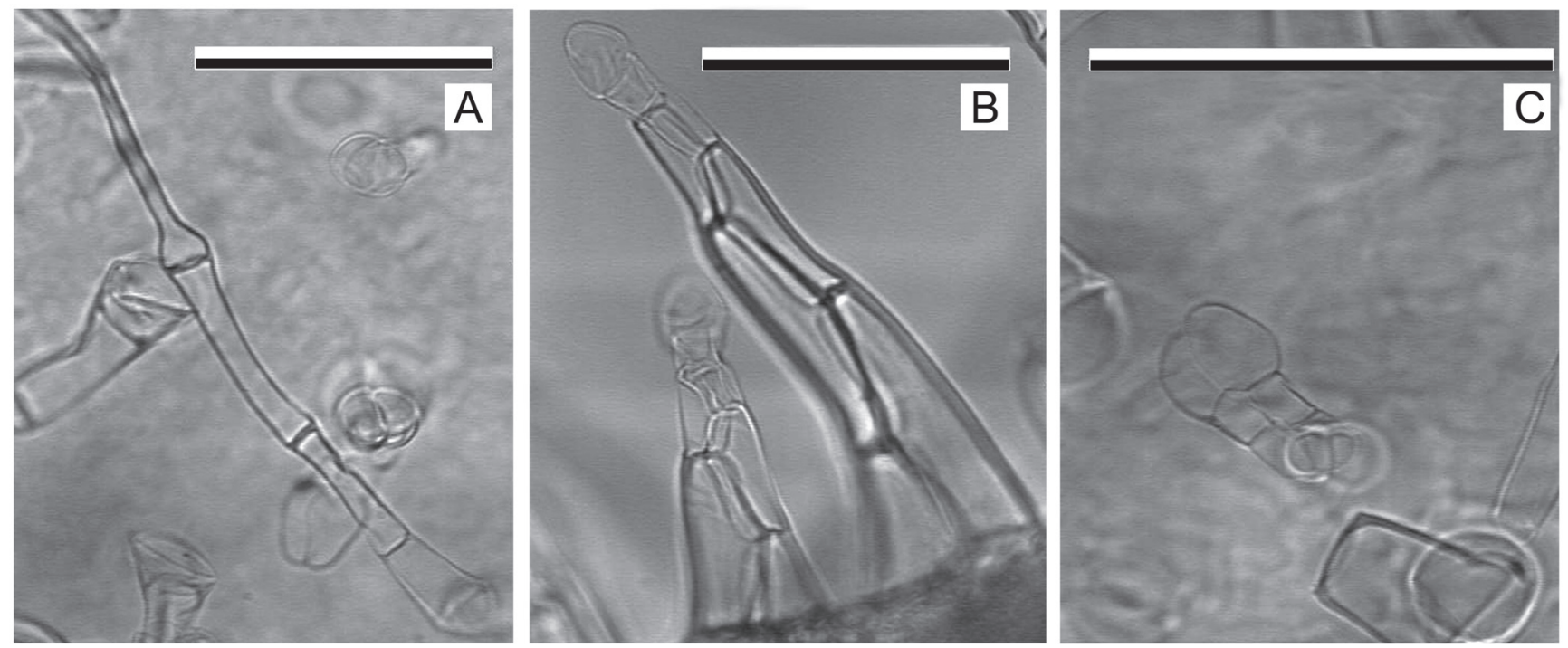

Figure 1. Foliar trichomes of Pseudognaphalium. A, Flagellate trichome (P. viravira, Teillier \& Márquez 5315, CONC); B, Long biseriate glandular trichomes (P. gayanum, Werdermann 470, SI); C, Short biseriate glandular trichome (P. gayanum, Werdermann 470, SI). Scale bars: $\mathrm{A}-\mathrm{C}=0.1 \mathrm{~mm}$.

Figura 1. Tricomas foliares de Pseudognaphalium. A, Tricoma flagelado (P. viravira Teillier \& Márquez 5315, CONC); B, Tricoma glandular biseriado largo (P. gayanum, Werdermann 470, SI); C, Tricoma glandular biseriado corto (P. gayanum, Werdermann 470, SI). Escalas: $\mathrm{A}-\mathrm{C}=0,1 \mathrm{~mm}$. 

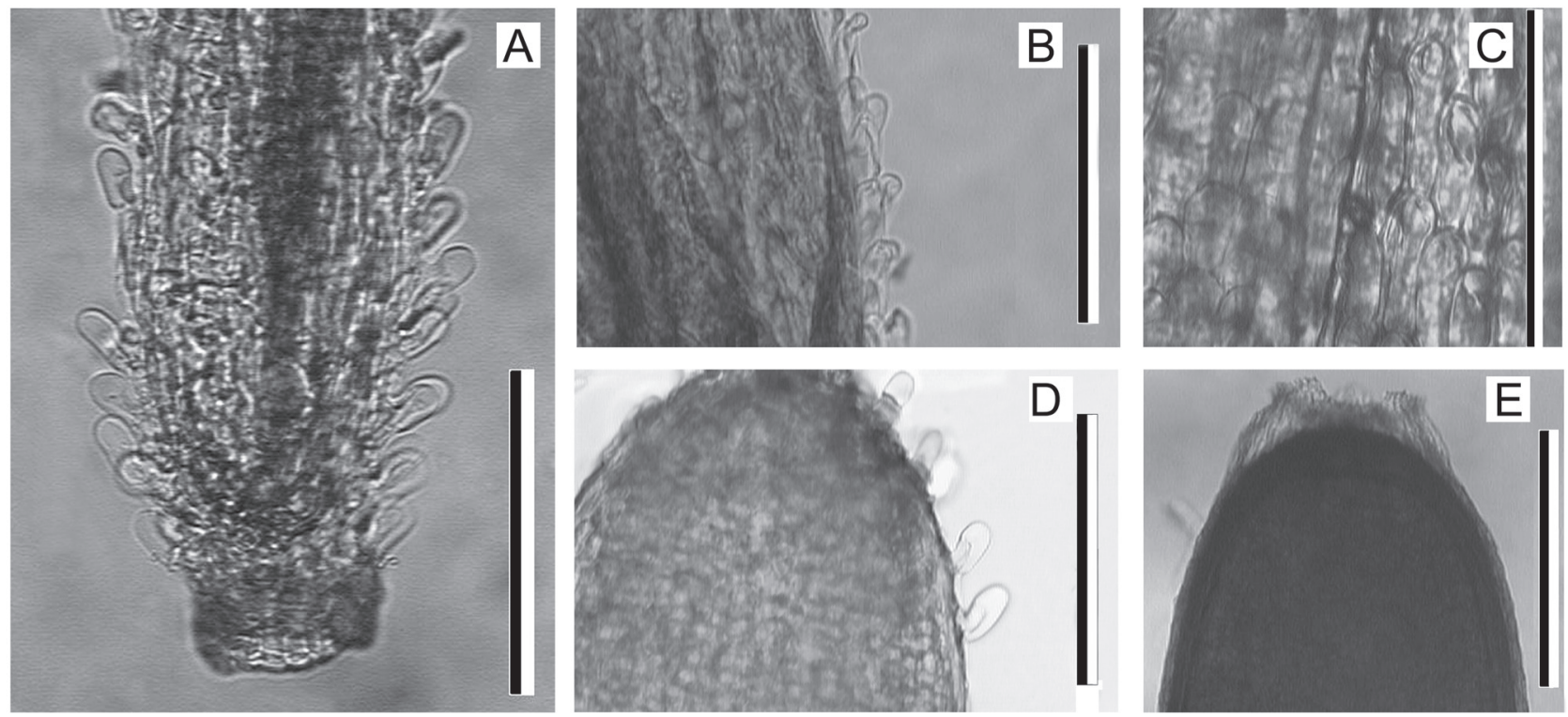

Figure 2. Achenes of Pseudognaphalium. A, Short pilose achene (P. aldunateoides, Urtubey et al. 700, SI); B, Achene with papillose epidermis (P. psilophyllum, Werdermann 1100, SI). C, Surface view of imbricate achenial papillae (P. viravira, Matte 745, CONC). D, Achene with short oblong duplex hairs (P. aldunateoides, Mihoc 6227, CONC). E, Glabrous achene with smooth epidermis and papillose epidermis at the apex (P. munoziae, Moreira et al. 1676, SGO). Scale bars: A-E= $0.1 \mathrm{~mm}$.

Figura 2. Aquenios de Pseudognaphalium. A, Aquenio cortamente piloso (P. aldunateoides, Urtubey et al. 700, SI); B, Aquenio con epidermis papilosa ( $P$. psilophyllum, Werdermann 1100, SI). C, Vista superficial de la epidermis del aquenio con papilas imbricadas $(P$. viravira, Matte 745, CONC). D, Aquenio con pelos dobles cortos y oblongos (P. aldunateoides, Mihoc 6227, CONC). E, Aquenio glabro con epidermis lisa, sólo papilosa en el ápice (P. munoziae, Moreira et al. 1676, SGO). Escalas: A-E= $0.1 \mathrm{~mm}$.
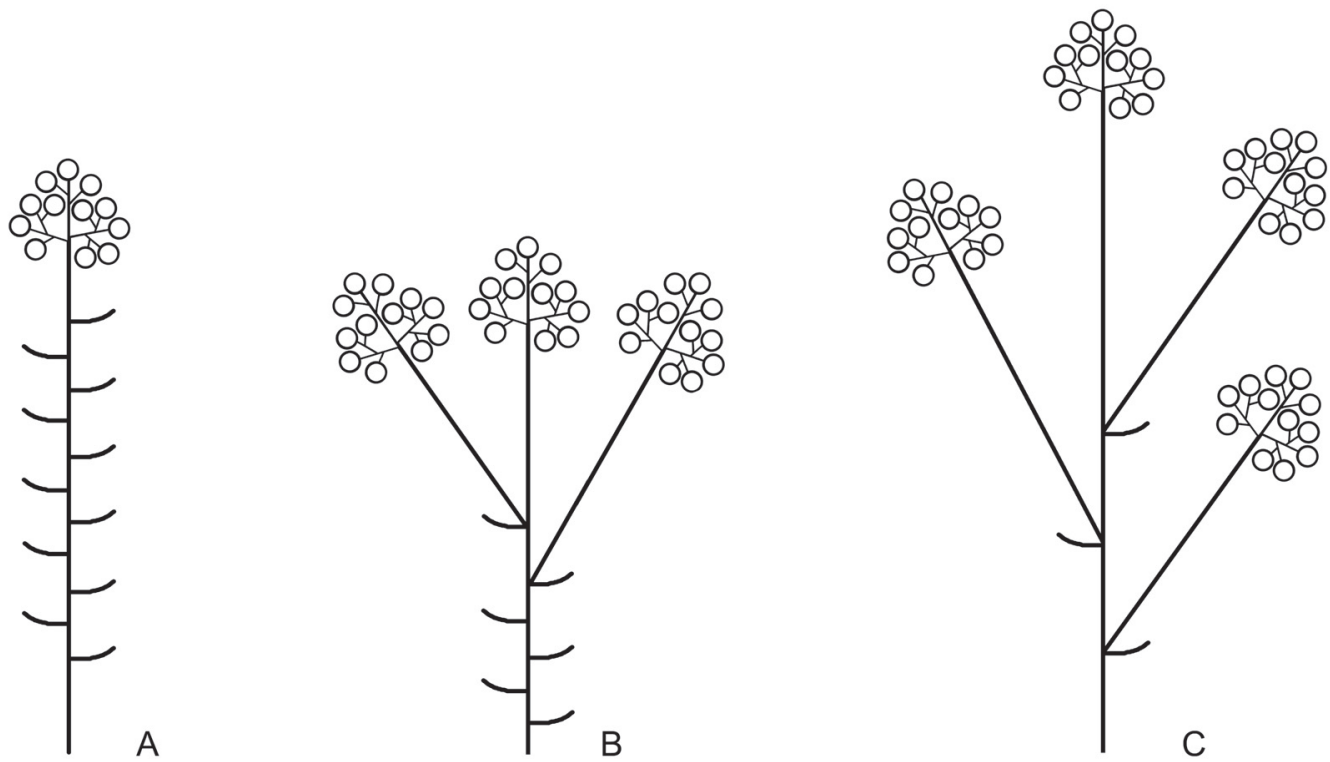

Figure 3. Inflorescences of Pseudognaphalium. A, Capitula arranged in dense terminal clusters (P. tarapacanum, $P$. viravira); B, Capitula in dense clusters arranged in corymbs ( $P$. aldunateoides, $P$. cheiranthifolium, $P$. cymatoides, $P$. cabrerae, $P$. gayanum, $P$. lacteum, $P$. landbeckii, P. psilophyllum, P. viravira); C, Capitula in dense clusters arranged in thyrsoid inflorescence (P. aldunateoides, $P$. cymatoides, P. gayanum, P. lacteum, P. munoziae, P. psilophyllum, P. remyanum).

Figura 3. Inflorescencia de Pseudognaphalium. A, Capítulos agrupados en densos glomérulos terminales (P. tarapacanum, P. viravira); $\mathrm{B}$, Capítulos en densos glomérulos reunidos a su vez en cimas corimbiformes $(P$. aldunateoides, $P$. cheiranthifolium, $P$. cymatoides, $P$. cabrerae, P. gayanum, P. lacteum, P. landbeckii, P. psilophyllum, P. viravira); C, Capítulos en densos glomérulos reunidos a su vez en inflorescencias tirsoides (P. aldunateoides, P. cymatoides, P. gayanum, P. lacteum, P. munoziae, P. psilophyllum, P. remyanum). 
Corolla. The corollas are whitish or yellowish. Corollas of pistillate florets are filiform and corollas of bisexual florets are tubular, scarsely broadened and glandulose above, 5-lobed, usually whitish or yellowish (purplish in $P$. aldunateoides).

ANTHERs. As well as most species of the tribe Gnaphalieae, all the taxa of Pseudognaphalium in this study have an apical ovate to lanceolate anther appendage and short basal tails (Fig. 4B).
StYle. As well as many taxa of the tribe Gnaphalieae (e.g. Achyrocline, Gamochaeta), all the species of Pseudognaphalium in this study have apically pilose styles, with the two branches truncate to rounded at the apex (Fig. 4C).

PAPPus. In all taxa studied pappus bristles are barbellate, with apical cells rounded and inflated, and bases cohering by patent cilia (Fig. 4D).

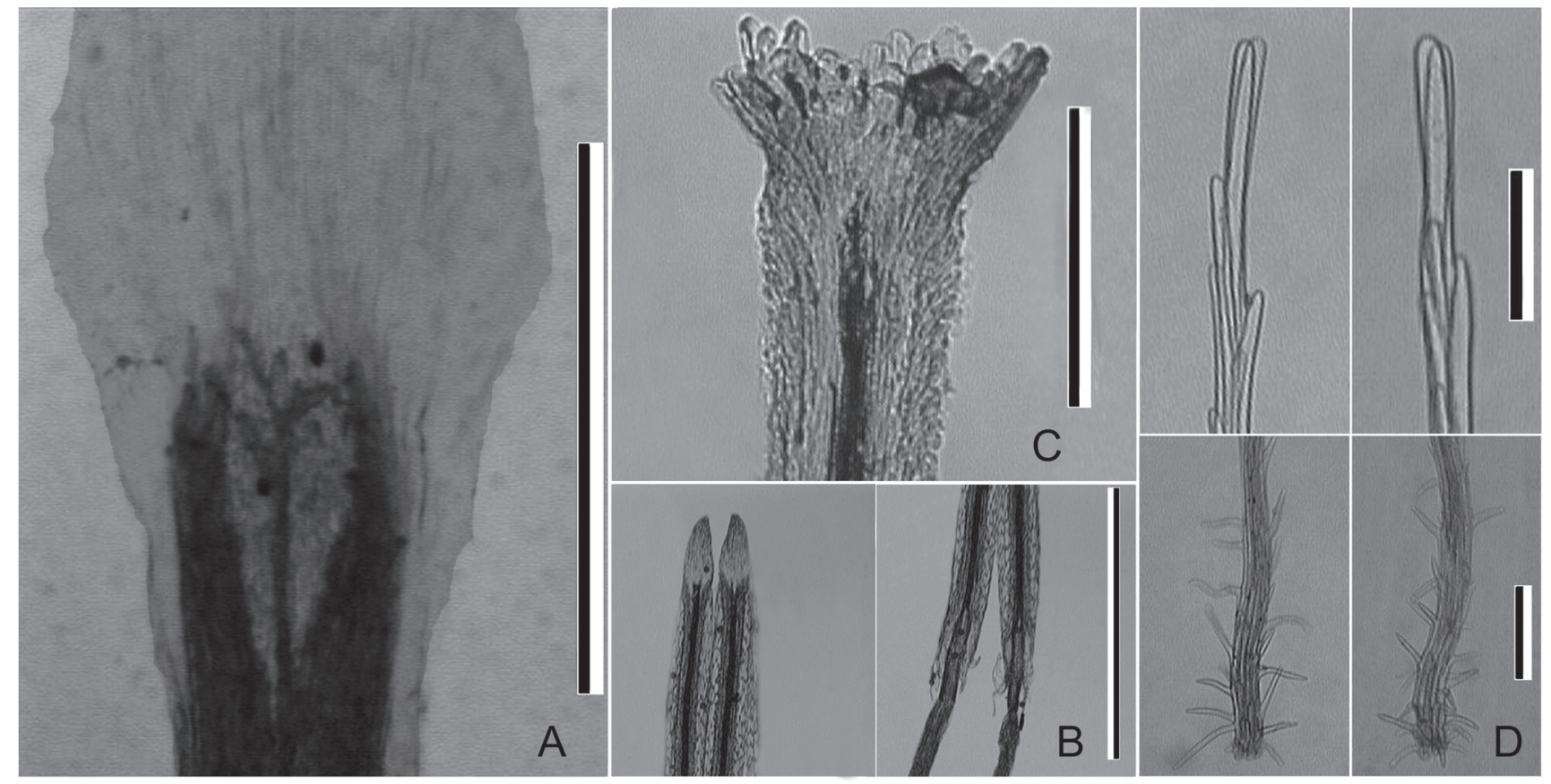

Figure 4. A, Phyllary showing the divided stereome (P. viravira, Barros 6076 LP); B, Anther (P. cheiranthifolium, Palma \& Inostroza s.n., CONC); C, Style branch from bisexual floret (P. cheiranthifolium, Palma \& Inostroza s.n., CONC); D, Pappus bristles (monomorphic) showing tip cells and basal portion, left to right: pappus bristle of female, pappus bristle of bisexual floret (P. munoziae, Moreira et al. 1676, SGO). Scale bars: A, B=1 mm, C, D=0.1 mm.

Figura 4. A, Filarios con esteroma dividido (P. viravira, Barros 6076 LP); B, Antera (P. cheiranthifolium, Palma \& Inostroza s.n., CONC); C, Ramas del estilo de una flor perfecta (P. cheiranthifolium, Palma \& Inostroza s.n., CONC); D, Pelos del papus (monomórfico) mostrando las células apicales y las cilias basales; a la izquierda: pelo del papus de una flor pistilada; a la derecha: pelo del papus de una flor perfecta (P. munoziae, Moreira et al. 1676, SGO). Escalas: A, B=1 mm, C, D=0.1 mm.

\section{TAXONOMIC TREATMENT}

Twelve species of Pseudognaphalium occur in Chile, representing about $15 \%$ of the known species for the genus.

Pseudognaphalium Kirp., Trudy Bot. Inst. Akad. Nauk S.S.S.R., Ser 1, Fl. Sist. Vyssh. Rast. 9: 33. 1950. TYPE: Gnaphalium oxyphyllum DC. = Pseudognaphalium oxyphyllum (DC.) Kirp.

Gnaphalium L., Sp. Pl. 2: 850. 1753, p.p. excl. type.

Hypelichrysum Kirp., Trudy Bot. Inst. Akad. Nauk SSSR,
Ser. 1, Fl. Sist. Vyssh. Rast. 9: 33. 1950. TYPE: Gnaphalium heterotrichum Phil. = Hypelichrysum heterotrichum (Phil.) Kirp. $=$ Pseudognaphalium heterotrichum (Phil.) Anderb.

Gnaphalium L. sect. Calolepis Kirp., Bot. Mater. Gerb. Bot. Inst. Komarova Akad. Nauk S.S.S.R. 20: 309. 1960. Pseudognaphalium subgen. Laphangium Hilliard \& B.L. Burtt, Bot. J. Linn. Soc. 82(3): 205. 1981. Laphangium (Hilliard \& B.L. Burtt) Tzvelev, Byull. Moskovsk. Obshch. Isp. Prir. Otd. Biol. 98(6): 105. 1993[1994].

Gnaphalium Kirp. subgen. Laphangium (Hilliard \& B.L. Burtt) P.D. Sell, Fl. Gr. Brit. Ireland 4: 555. 2006. 
TYPE: Gnaphalium luteoalbum L. = Pseudognaphalium luteoalbum (L.) Hilliard \& B.L. Burtt.

Annual, biennial or perennial herbs, woolly or glandularwoolly. Leaves alternate, entire, sessile, often stem-clasping and decurrent. Capitula small, heterogamous, discoid, sessile or short pedunculate, in small clusters arranged in corymbs or panicles. Involucre often campanulate; phyllaries in 3-4-series, papery, phyllary lamina monochromous opaquewhite or hyaline and shiny, stereome divided. Receptacle smooth or honeycombed. Florets many, pistillate florets outnumbering the bisexual; pistillate corollas filiform or narrowly tubular; bisexual corollas tubular, scarsely broaded above, 5-lobed, all corollas yellowish or whitish, lobes glandular and often yellowish or whitish. Anthers with a small obtuse apical anther appendage; tails slightly longer or shorter than the filament collar. Style branches truncate and penicillate. Achenes glabrous often with imbricate microcopic papillae or pilose with short duplex myxogenic hairs. Pappus bristles monomorphic, barbellate, apical cells sometimes inflated, bases cohering by patent cilia. Chromosome number: 2n = 14 (Turner, 1970; Mehra \& Remanandan, 1975; Keil, 1981; Groves, 1977); 2n = 28 (Keil \& Stuessy, 1977).

Worldwide distribution, mostly American, some African, and Asian species. About 90 species of which twelve grow in Chile between sea level and $4500 \mathrm{~m}$.

\section{Key to the species of PSeudognaphalium in Chile}

1. Dwarf herbs; stems prostrate, branched.

P. lacteum

1'. Dwarf or large herbs; stems erect or ascending, branched or unbranched.

2. Basal leaves rosulate, longer than the upper leaves; upper leaves alternate. Stems $4-6(15) \mathrm{cm}$ tall. ................................P. munoziae

2'. Basal and upper leaves alternate.

3. Leaf blade margins conspicuously undulate.

4. Weak stems 7-50 cm tall, branched; involucre $2-3 \mathrm{~mm}$ high

P. cymatoides

4 . Robust stems $30-110 \mathrm{~cm}$ tall, unbranched; involucre $4-5 \mathrm{~mm}$ high.

3'. Leaf blade margins flat (occasionally some with undulate margins).

5. Leaves glandular or arachnoid-glandular.

6. Upper leaves broad at base.

6'. Upper leaves not broad at base.

.P. tarapacanum

5'. Leaves usually whitish-woolly with short glandular trichomes hidden under the woolly trichomes (rarely with long glandular trichomes raised over woolly trichomes).

7. Dwarf plants (stems 1-10 cm). Capitula 3-5(7) arranged in corymbs or narrow panicles. Achenes short pilose

7'. Usually large plants; stems $>10 \mathrm{~cm}$ tall (when less than $10 \mathrm{~cm}$ tall, basal leaves approximate). Capitula numerous. Achenes glabrous or short pilose.

8. Caulinar leaves oblong, apex obtuse.

P. landbeckii

8'. Caulinar leaves linear, linear-lanceolate, lanceolate or linear-obovate, apex acute.

9. Leaves linear-lanceolate or lanceolate.

10. Stem and leaves whitish, woolly with long glandular trichomes raised over the woolly trichomes. Leaves concolorous.

10'. Stems and leaves whitish, woolly, with short glandular trichomes hidden under the woolly trichomes. Leaves slightly discolorous to discolorous.

P. cabrerae

$9^{\prime}$. Leaves linear or linear-obovate.

11. Stem branched above the base. Leaves arachnoid, adaxial surface green. Capitula arranged in corymbs or lax panicles.

P. remyanum

11'. Multistemmed plants with unbranched stems. Leaves usually whitish woolly. Capitula usually arranged in dense terminal clusters.

P. viravira

1. Pseudognaphalium aldunateoides (J. Rémy) C. Monti, N. Bayón \& S.E. Freire, J. Bot. Res. Inst. Texas 7(1): 196. 2013. Gnaphalium aldunateoides J. Rémy, in Gay Fl. Chil. 4(2): 232. 1849. TYPE: Chile. 'Se cría en los cerros de Santiago y florece en setiembre [Gay]' (P). Fig. 5.

Gnaphalium insulare Phil., Bot. Zeitung (Berlin) 14: 645. 1856. TYPE: CHILE. Islas de Juan Fernández, Más Afuera, X-1854, Germain s.n. (holotype, SGO 64416!; isotypes, B, probably destroyed, = photo F15103!, LP 001926 fragment ex SGO!).

Gnaphalium perpusillum Phil., Linnaea 29: 6. 1858, syn. nov. Pseudognaphalium perpusillum (Phil.) C. Monti, N. Bayón \& S.E. Freire, J. Bot. Res. Inst. Texas 7(1): 197. 2013. TYPE: CHILE. Coquimbo: Sotaqui, Jan 1837, C. Gay 739 (holotype, SGO 64448!). 

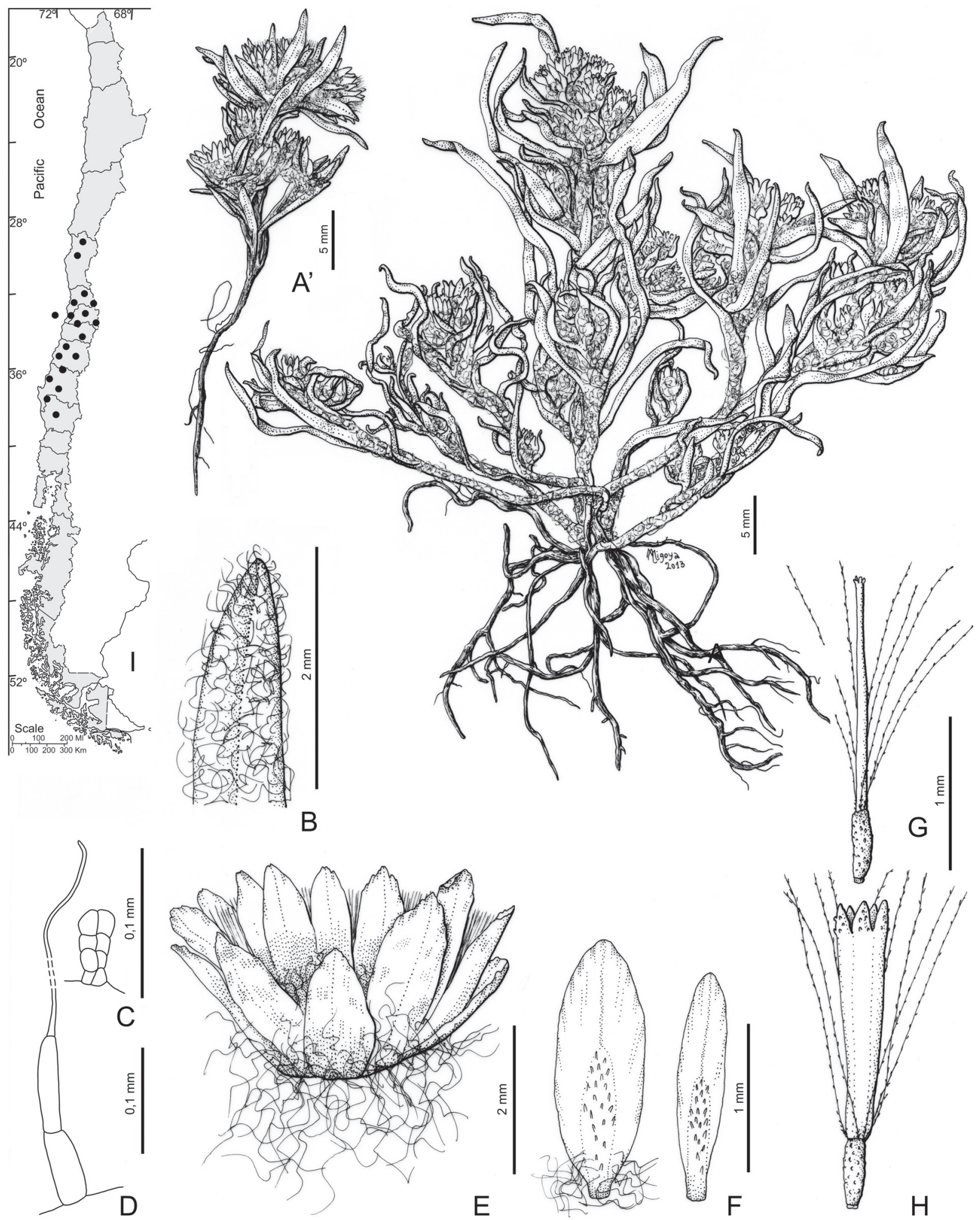

Figure 5. Pseudognaphalium aldunateoides. A, A', Habits; B, Leaf pubescence; C, D, Foliar trichomes; E, Capitulum; F, Phyllaries; G, Pistillate floret; H, Bisexual floret. I, Distribution [A, C-E, H, Urtubey et al. 700, SI; A', B, Boelcke 2414, LP; F, Ricardi 5445, CONC].

Figura 5. Pseudognaphalium aldunateoides. A, A', Plantas; B, Pubescencia de la hoja; C, D, Tricomas foliares; E, Capítulo; F, Filarios; G, Flor pistilada; H, Flor perfecta. I, Distribución [A, C-E, H, Urtubey et al. 700, SI; A', B, Boelcke 2414, LP; F, Ricardi 5445, CONC]. 
Gnaphalium phaeolepis Phil., Linnaea 33: 168. 1864. TYPE: Chile. without locality, year 1865, Frumau 63 (lectotype designated by Monti et al. (2013: 196), SGO 64452!; isolectotypes, B (probably destroyed), = photo F15112!, SGO 44946!, 44947!).

Gnaphalium diminutivum Phil., Linnaea 33: 167. 1864. syn. nov. TYPE: Chile. Colchagua, Cerro del Volcán [ad originem fluvii Tinguiguirica], II-1861, W. Diaz s.n. (lectotype designated by Monti et al. (2013: 197), SGO 64454!; isolectotype SGO 44923!).

Dwarf annual herbs, 1-10 cm tall, multistemmed (single stem in very small plants), stems erect or ascending, usually unbranched, lanuginose. Stem leaves 6-16 × 1-3(4) mm, linear or linear-oblong, margin flat, apex acute or subobtuse, mucronate; basal leaves $8-9 \times 2.5-3 \mathrm{~mm}$; concolorous, whitish-woolly on both faces, trichomes of 2 types, long eglandular eseptate, 3-8-celular, and few short glandular biseriate trichomes, hidden under the wool. Capitula few, $3-5(7)$ in clusters arranged in corymbs or narrow panicles, clusters subtended by linear leaves. Involucre campanulate, 3-3.2 × 2.5-3 mm. Phyllaries 3-4-seriate, lamina opaque white or hyaline whitish at apex; outer phyllaries 1.8-2.8 $\times 0.6-1 \mathrm{~mm}$, ovate, apex obtuse to acute; inner phyllaries 1.5-3 × 0.4-0.9 mm, linear-obovate, apex acute. Pistillate florets 96-150, corolla white-yellowish, purplish at apex, filiform, 0.9-1.7 mm long. Bisexual florets 4-13, corolla white-yellowish, purplish at apex, tubular, 1-1.7 mm long. Achenes 0.3-0.6 mm long, pilose, with short oblong duplex hairs. Pappus bristles $1-1.8 \mathrm{~mm}$ long.

Phenology. Flowering and fruiting from October to March.

Distribution and habitat. Central and South Chile (Coquimbo, Valparaíso, Metropolitana, Libertador General Bernardo O'Higgins, Archipiélago Juan Fernández, Maule, Biobío, Araucanía) and West Argentina (Chubut, Mendoza, Neuquén, Río Negro, San Juan), growing in mossy places and river margins, from sea level to $3700 \mathrm{~m}$.

Relationships. Pseudognaphalium aldunateoides is easily distinguished by its dwarf habit with erect to ascending unbranched stems, linear leaves, few capitula in clusters arranged in corymbs or narrow panicles, and short pilose achenes.

\section{OBSERVATIONS.}

1. According to the protologue, Gnaphalium aldunateoides was based on the specimen. 'Se cría en los cerros de Santiago y florece en setiembre'. We found at $\mathrm{P}(00704557,=$ photo! $)$ a collection, 'Stiago. (Chili), Sbre. 1839, C. Gay 936' which is in accordance with the protologue (although no collection number is mentioned there) and has the annotation 'Type'.
Since we were able to see only one sheet, we prefer not to select lectotype in this case.

2. Klatt (Linnaea 42: 121. 1878) mentioned Gnaphalium rivulare, as a synonym of $G$. insulare Phil. According to Philippi (Anales Univ. Chile 90: 18. 1895) it is a misinterpretation by Klatt, who express that he found at Berlin herbarium two specimens collected at Masafuera island by Philippi, one named $G$. rivulare and the other one G. insulare. Philippi (1.c.) stated that Klatt probably read 'riv' instead of 'ins.'

3. Gnaphalium perpusillum Phil. was described on the basis of its dwarf plants ca. $1.5 \mathrm{~cm}$ tall with single stem. However, this is a variable feature throughout the range of G. aldunateoides.

4. Gnaphalium diminutivum Phil. which was previously placed into synonymy of $G$. perpusillum Phil. (Monti et al. 2013) is here considered as synonym of Pseudognaphalium aldunateoides.

Additional material examined. CHILE. Región de Coquimbo. Prov. Elqui, Paihuano, III-1946, Roesner 6508 (CONC); Prov. Limarí, Ovalle, Tulahuén, 14-I-1942, Barros 2258 (LP). Región de Valparaíso. Prov. Los Andes, Comuna de Los Andes, cajón del río Colorado, vega de Lagunillas, 32³6'S, 70²0’W, 20-II-2002, Teillier 51112 (CONC); Potrero Escondido, 22-II-1947, 3500 m, Boelcke 2424 dupl. (LP); Prov. San Felipe de Aconcagua, Putaendo, Laguna de Copín, 2620 m, 26-I-1955, Parra 37 (CONC); Prov. Valparaíso, año 1891, Cuming s.n. (LP), Lago Peñuelas, 7-II-1968, Ricardi 5445 (CONC), Placilla, 3307'S, 71³5’W, 28-XII-1993, Teillier 3224 (CONC). Región Metropolitana de Santiago. Prov. Cordillera, San José del Maipo, Cajón del Morales, entre la laguna y el glaciar, 27I-2002, Teillier \& Márquez 5316 (CONC); Prov. Melipilla, Alhué, Monte Cantillana, 2-I-1939, Barros 2272 (LP); Prov. Santiago, vega entre Casa de Piedra Carvajal y Cerro La Paloma, sector alto del estero de la Yerba Loca, 33 13 '04' S, 70¹6'18.2”W, 14-II-1999, Arroyo \& Humaña 991714 (CONC); Bajando de La Parva, 2859 m, 24-I-2012, Urtubey et al. 700 (SI). Región del Maule. Prov. Cauquenes, ca. 2 km south of Chanco on west side of road, $35^{\circ} 45^{\prime}$ S, $72^{\circ} 32^{\prime} \mathrm{W}, 50$ m, 22-XI-1995, Bliss 2272 (CONC); Prov. Linares, Quella Sur, 36 $05^{\prime} \mathrm{S}, 7^{\circ} 08^{\prime} \mathrm{W}, 21-\mathrm{I}-1991$, Matthei \& Quezada 1420 (CONC); Prov. Talca, Curepto, Puninque, 21-I-1926, Barros 34 (LP); Curepto, 10 m, I-1925, Barros 649 (CONC); Cordillera de Talca, El Picazo, 26-I-1939, Barros 2290 (LP). Región del Biobío. Prov. Arauco, Contulmo, cerros orillas L. Lanalhue, 30-XII-1949, Ricardi 9259 (CONC); Prov. Biobío, Nacimiento, 26-XII-1930, Barros 45 (LP); Negrete, a orillas del Bío-Bío, en la ribera norte del río, 19-II-1951, Pfister 10502 (CONC); Prov. Concepción, Hualpén, 9-III- 
1940, Junge 6767 (CONC); Prov. Ñuble, Ninhue, Río Ninhue, km 32 desde San Gregorio a Pocillas, $190 \mathrm{~m}$, $36^{\circ} 08^{\prime} \mathrm{S}, 7^{\circ} 05^{\prime} \mathrm{W}, 4-\mathrm{II}-2003$, Mihoc et al. 6227 (CONC). Región de la Araucanía. Prov. Cautín, Metrenco, 85 m, XI1946, Gunckel 46993 (CONC); Temuco, Río Cautín, 1-XII1957, Montero 5728 (CONC, LP).

2. Pseudognaphalium cabrerae (S.E. Freire) S.E. Freire, N. Bayón, C. Baeza, Giuliano \& C. Monti, comb. nov.

Basyonym: Gnaphalium cabrerae S.E. Freire, Flora Fanerogámica Argentina 14, Errata et corrigenda 4. 2000. Gnaphalium fastigiatum Phil., Anales Univ. Chile 90: 15. 1895, nom. illeg., non Thunb., 1800 nec Schrank, 1824. Gnaphalium philippii Cabrera, Revista Mus. La Plata, Secc. Bot. 4: 164. 194, nom. illeg., non Gandoger, 1918. Gnaphalium cabrerae S.E. Freire, Monogr. Syst. Bot. Missouri Bot. Gard. 74(2): 1245. 1999, nom. inval. Pseudognaphalium cabrerae (Freire) Deble, Balduinia 6: 29. 2006, comb. inval. Pseudognaphalium fastigiatum N. Bayón, Bol. Soc. Argent. Bot. 48 (3-4): 599. 2013, nom. illeg. TYPE: Chile. Santiago, Quinta Normal, III-1878, R.A. Philippi s.n. (holotype, SGO 64449!; isotype, LP 001914!).). Fig. 6.

Annual herbs, 40-60 cm tall, stem usually solitary, erect, rarely branched at the upper part, whitish-woolly. Leaves 35-70 × 4-7 mm, lanceolate, margin flat, apex longattenuate, acute, base decurrent 7-8 mm long; slightly discolorous to discolorous, with upper surface lanuginose and lower surface densely tomentose, trichomes of 2 types, long eglandular eseptate, 3-4-celular, and few short biseriate glandular trichomes hidden under the wool. Capitula numerous in clusters arranged in corymbs. Involucre broadly campanulate, 3-4 × 3-4 mm. Phyllaries 4-seriate, lamina straw-coloured to brownish, hyaline and shiny, apex obtuse; outer phyllaries 3-3.2 × 1.5-1.6 mm, ovate; inner phyllaries 3-3.3 $\times 0.5-0.8 \mathrm{~mm}$, oblong-obovate. Pistillate florets ca. 198, corolla yellow, filiform, $3.2 \mathrm{~mm}$ long. Bisexual florets ca. 11, corolla yellow, tubular, ca. $3.2 \mathrm{~mm}$ long. Achenes ca. $0.5 \mathrm{~mm}$ long, glabrous, epidermis papillose. Pappus bristles ca. $2.8 \mathrm{~mm}$ long.

Phenology. Flowering from November to February.

Distribution and habitat. Central Argentina (Buenos Aires, Catamarca, Córdoba, La Rioja, Mendoza, San Juan, San Luis) and North, Central Chile (Tarapacá, Metropolitana, Maule, Araucanía), growing from sea level to $2000 \mathrm{~m}$.

Relationships. Pseudognaphalium cabrerae is closely related to $P$. cheiranthifolium. Both species are woolly plants with usually solitary stems, linear-lanceolate or lanceolate leaf blades and numerous capitula in clusters arranged in corymbs. Pseudognaphalium cabrerae can be distinguished by its woolly leaves with short glandular trichomes hidden under the wool (vs. woolly leaves with long glandular trichomes raised over the wool in $P$. cheiranthifolium).

OBSERVATION

Recently, Bayón \& Giuliano (2013) proposed the name Pseudognaphalium fastigiatum N. Bayón as the correct name for Gnaphalium fastigiatum Phil. (hom. illeg.) when transferred to the genus Pseudognaphalium. However, according to ICN (McNeill et al. 2012), Art.11.4., the correct name is $P$. cabrerae, since the earliest legitimate name of the taxon is Gnaphalium cabrerae S.E. Freire, Flora Fanerogámica Argentina 14, Errata et Corrigenda 4. 2000. Since the name Pseudognaphalium cabrerae (S.E. Freire) Deble is not validly published because it was based on Gnaphalium cabrerae S.E. Freire, Monogr. Syst. Bot. Missouri Bot. Gard. 74(2): 1245. 1999 (nom. inval.), we propose the new combination Pseudognaphalium cabrerae (S.E. Freire) S.E. Freire, N. Bayón, C. Baeza, Giuliano \& C. Monti, based on Gnaphalium cabrerae S.E. Freire, Flora Fanerogámica Argentina 14, Errata et Corrigenda 4. 2000.

Additional material EXamined. CHILE. Región de Tarapacá. Prov. El Tamarugal, Termas de Mamiña, 15-IX-1981, 2900 m, Montero 12045 (CONC). Región Metropolitana de Santiago. Cerro Manquehue (Canal Lo Curro), 7-XII-1976, Elgueta 546 (CONC). Región del Maule. Prov. Curicó, Potrero Grande, 4-I-1927, Barros 1940 (CONC). Región de la Araucanía. Prov. Malleco, Lonquimay, 20-II-1936, Atanasio 706 (CONC).

3. Pseudognaphalium cheiranthifolium (Lam.) Hilliard \& B.L. Burtt, Bot. J. Linn. Soc. 82(3): 205. 1981. Gnaphalium cheiranthifolium Lam., Encycl. [J. Lamarck \& al.] 2(2): 752. 1788. TYPE: [Uruguay]. 'Commerson à trouvé cette plante à Monte-Video’ (P-Lam.?). Fig. 7.

Gnaphalium citrinum Hook. \& Arn., Bot. Beechey Voy.: 31. 1830. TYPE: Chile. 'Hab. Concepción' (holotype, probably $\mathrm{K}$; isotype, $\mathrm{SGO} 72232$ !).

Gnaphalium acutifolium Phil., Anales Univ. Chile 90: 12. 1895. TYPE: Chile. Arauco. Curanilahue I-1893, Philippi s.n. (lectotype, here designated, SGO 64401!; isolectotypes, SGO 35992!, 44968!).

Gnaphalium araucanum Phil., Anales Univ. Chile 43: 502. 1873. TYPE: Chile. Biobío: Lebu, G. Volkmann s.n. (lectotype, here designated, SGO 71287!; isolectotype, SGO 71281!). 

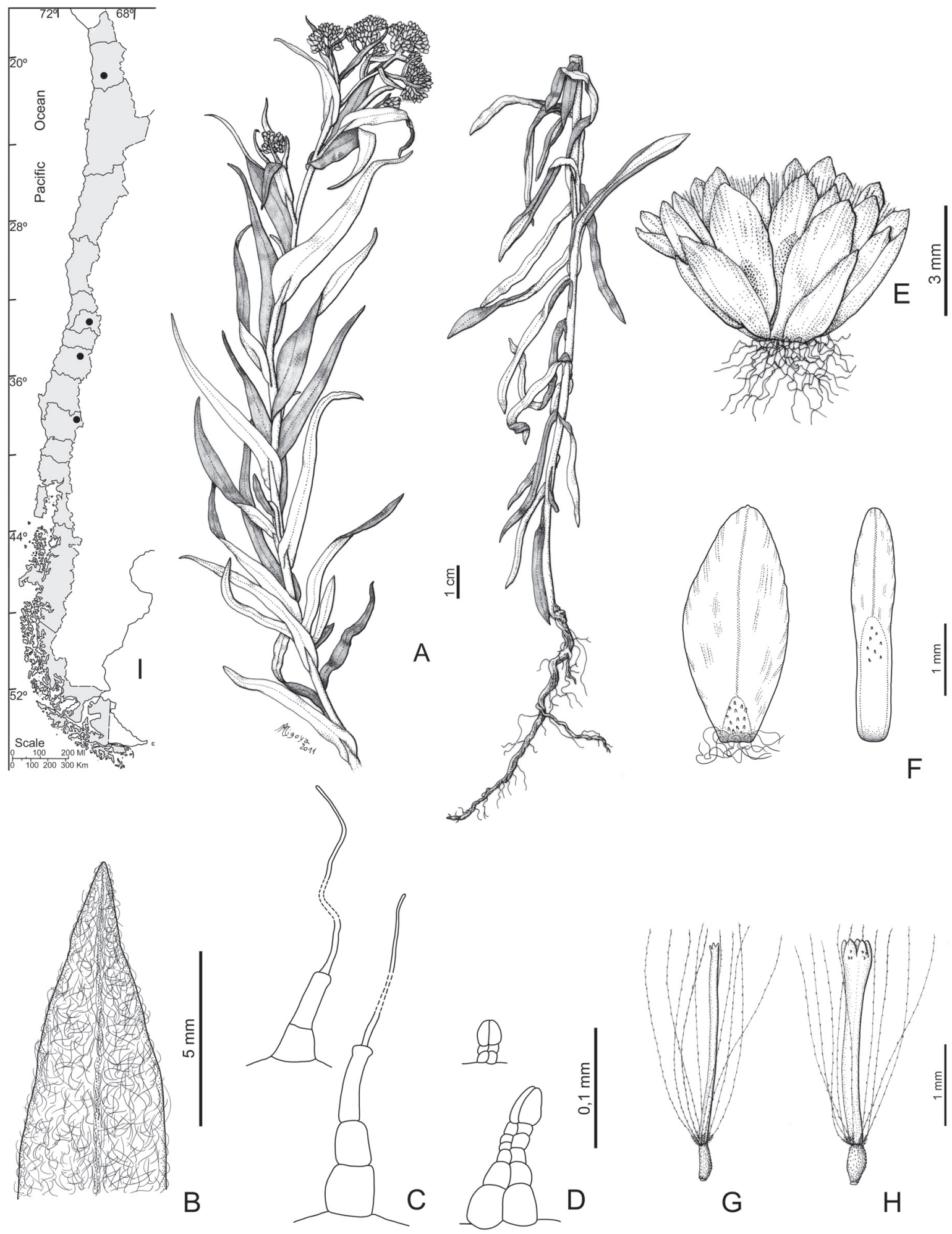

Figure 6. Pseudognaphalium cabrerae. A, Habit; B, Leaf pubescence; C, D, Foliar trichomes; E, Capitulum; F, Phyllaries; G, Pistillate floret; H, Bisexual floret. I, Distribution [A, E, G, H, Barlett 20018, SI; C, D, Cabrera 187, LP; B, F, Cabrera 5189, LP].

Figura 6. Pseudognaphalium cabrerae. A, Planta; B, Pubescencia de la hoja; C, D, Tricomas foliares; E, Capítulo; F, Filarios; G, Flor pistilada; H, Flor perfecta. I, Distribución [A, E, G, H, Barlett 20018, SI; C, D, Cabrera 187, LP; B, F, Cabrera 5189, LP]. 

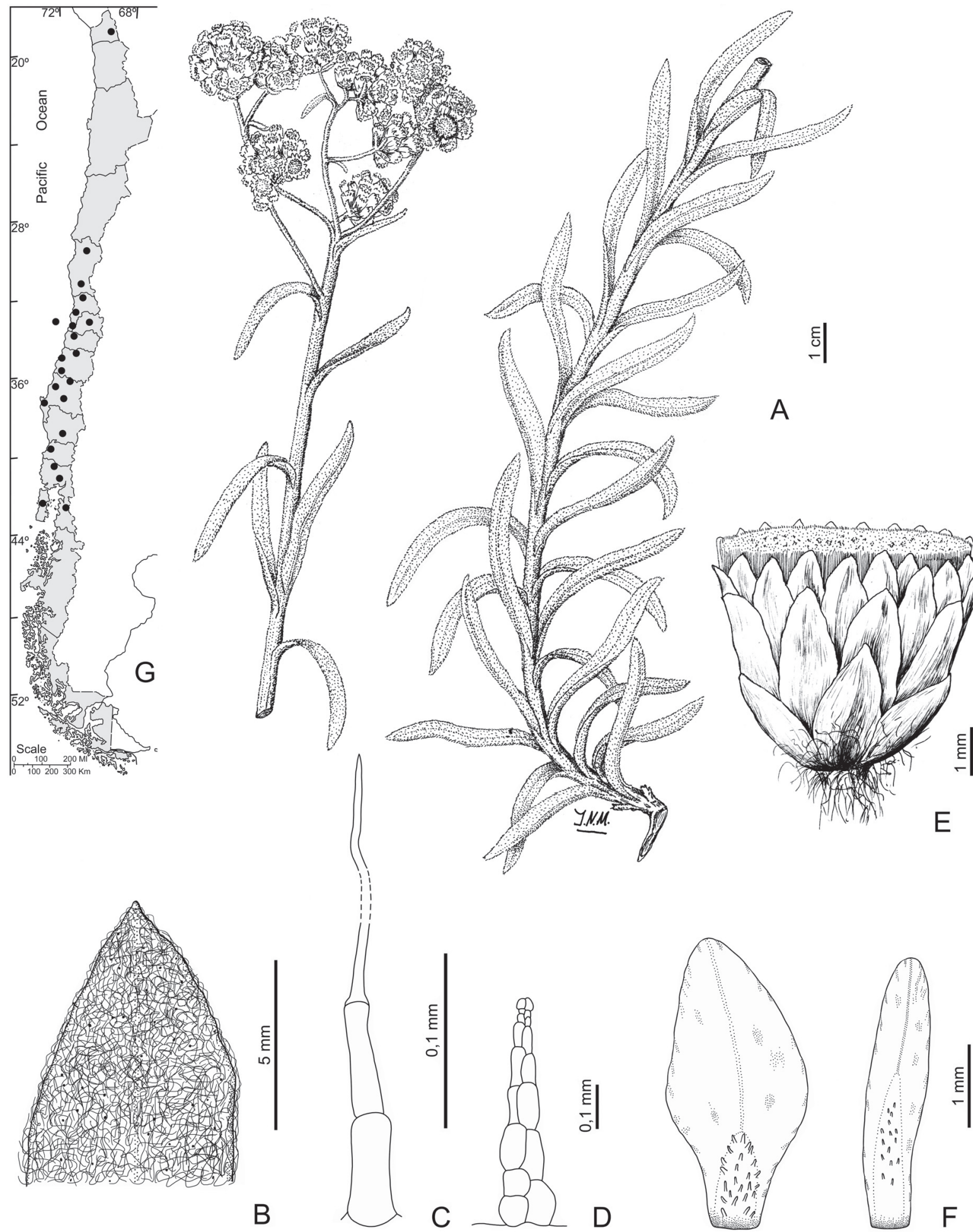

Figure 7. Pseudognaphalium cheiranthifolium. A, Habit; B, Leaf pubescence; C, D, Foliar trichomes; E, Capitulum; F, Phyllaries. G, Distribution [A, E, from Cabrera 1978; B-D, Palma \& Inostroza s.n., CONC; F, Finot \& Leppe 97, CONC].

Figura 7. Pseudognaphalium cheiranthifolium. A, Planta; B, Pubescencia de la hoja; C, D, Tricomas foliares; E, Capítulo; F, Filarios; G, Distribución [A, E, tomado de Cabrera 1978; B-D, Palma \& Inostroza s.n., CONC; F, Finot \& Leppe 97, CONC]. 
Gnaphalium paniculatum Bertero ex Colla, Mem. Reale Accad. Sci. Torino 38: 17. 1835. Gnaphalium cheiranthifolium Lam. var. paniculatum (Bertero ex Colla) Skottsb., Kongl. Svenska Vetenskapsakad. Handl. 51(9): 5. 1914. TYPE: [Chile] 'Cultum in H. Ripul. e seminibus missis a BERTERO sine nomine specifico et lectis in saxosis apricis Valparaiso, servatur hyeme in frigidario, et floret secundo anno, ac raro diutius perdurat.' (holotype, probably $\mathrm{TO}$, not seen).

Gnaphalium valdivianum Phil., Linnaea 29: 6. 1858. TYPE: Chile. Valdivia, IV-1852, s. leg. (holotype, SGO 64374!).

Gnaphalium cheiranthifolium Lam. var. multiflorum J. Koster, Blumea 5(3): 655. 1945. TYPE: Bolivia. Samaipata, 2000-2200 m, III-1911, T. Herzog 1758 (holotype, L, = phot!; isotypes, B 10 0093571, = phot!, LP 001906!).

Perennial herbs, $40-80 \mathrm{~cm}$ tall, stem solitary ( 2 or 3 ), erect, unbranched or branched at the upper part, woollyglandulose. Stem leaves 30-45 × 2-5 mm, lanceolate or linear-lanceolate, margin flat, apex long-attenuate, acute, base decurrent 6-13 mm long; basal leaves $40-80 \times 3-8$ $\mathrm{mm}$, oblanceolate-spathulate, apex obtuse; concolorous, adaxial surface glandulose-tomentose, trichomes of 2 types, long eglandular eseptate, 3-celular, and long glandular biseriate trichomes raised over the wool, abaxial surface tomentose. Capitula numerous in clusters arranged in corymbs. Involucre broadly campanulate, $4-4.5 \times 5-6 \mathrm{~mm}$. Phyllaries 4-seriate, lamina yellow-greenish, hyaline and shiny; outer phyllaries 3.8-4 × 1.7-1.8 mm, ovate, apex subobtuse; inner phyllaries $4.3-4.5 \times 0.6-1 \mathrm{~mm}$, oblongobovate, apex acute. Pistillate florets 78-80, corolla yellow, filiform, ca. $3 \mathrm{~mm}$ long. Bisexual florets $10-15$, corolla yellow, tubulose, ca. $3 \mathrm{~mm}$ long. Achenes $0.7-0.8 \mathrm{~mm}$ long, glabrous, epidermis smooth or papillose. Pappus bristles ca. $3 \mathrm{~mm}$ long.

Phenology. Flowering from October to April.

Vernacular name. 'Té de burro'.

Distribution And habitat. Southern South America, i.e. southern Brazil (Paraná, Santa Catarina; Freire et al. 2011), Bolivia (Hind 2011), Uruguay (Rocha, San José), Argentina (Buenos Aires, Córdoba, Entre Ríos, Jujuy, La Pampa, La Rioja, Salta, San Luis), and Chile (Arica y Parinacota, Coquimbo, Valparaíso, Metropolitana, Libertador General Bernardo O'Higgins, Maule, Biobío, Araucanía, Los Lagos, Los Ríos, Archipiélago Juan Fernández). Commonly collected on rocky and sandy soils, from sea level to $2500 \mathrm{~m}$.

Relationships. Pseudognaphalium cheiranthifolium is closely related to $P$. cabrerae. Both species are plants with usually solitary stems, linear-lanceolate or lanceolate leaf blades and numerous capitula in clusters arranged in corymbs. Pseudognaphalium cheiranthifolium can be distinguished by its concolorous woolly leaves with long glandular trichomes raised over the wool (vs. slightly discolorous to discolorous leaves, lanuginose on adaxial surface and densely tomentose on abaxial surface, with short glandular trichomes hidden under the wool in P. cabrerae).

\section{OBSERVATIONS}

1. According to the protologue, Gnaphalium cheiranthifolium Lam., was based on the specimen 'Commerson à trouvé cette plante à Monte-Video'. We found at $\mathrm{P}$ the collection Commerson 103, P 00704563, which is in accordance with the protologue (although no collection number is mentioned there). However, since we were unable to locate this specimen at Lamarck's herbarium, we prefer not to select lectotype in this case.

2. According to the protologue, Gnaphalium araucanum Phil., was based on the specimen 'don Jerman Volkmann halló esta especie cerca de Lebu, en la Araucania'. Muñoz Pizarro (1960) cited SGO 64381 as the holotype. However, only two other sheets 'Lebu, Volkmann s.n.' were located at SGO, SGO 71281 and SGO 71287. We selected as lectotype the specimen SGO 71287, which is better preserved.

3. According to the protologue, Gnaphalium paniculatum Bertero ex Colla, was based on the specimen 'Cultum in H. Ripul. e seminibus missis a BERTERO sine nomine specifico et lectis in saxosis apricis Valparaiso, servatur hyeme in frigidario, et floret secundo anno, ac raro diutius perdurat.' We located three sheets, P 00704559, P 00704560, P 00704561, and one at LP 001936! ex P of the specimen 'Valparaiso, XII-1829, Bertero 1033', which are in accordance with the protologue (although no collection number and date are mentioned there). Since we were unable to see the Colla's original material probably kept at $\mathrm{TO}$, we prefer not to select lectotype in this case.

Additional material examined. CHILE. Región de Arica y Parinacota. Prov. Parinacota, camino entre Putre y Socoroma, km 8, 3600 m, 18 $8^{\circ} 12^{\prime} \mathrm{S}, 6^{\circ} 35^{\prime} \mathrm{W}, 21-\mathrm{III}-1987$, Matthei \& Rodríguez 299 (CONC). Región de Coquimbo. Prov. Choapa, Carretera Panamericana, $7 \mathrm{~km} \mathrm{~N}$ de Los Vilos, cerca de Agua Amarilla, $15 \mathrm{~m}, 31^{\circ} 51^{\prime} \mathrm{S}, 71^{\circ} 30^{\prime} \mathrm{W}$, 2-XI-1974, C. Marticorena et al. 353 (CONC); Pichidangui, 30 m, 19-II-1966, Mahu 10335 (LP); Prov. Elqui, Quebrada Las Trancas, $25 \mathrm{~km} \mathrm{~S}$ of Vicuña, $1350 \mathrm{~m}, 16-\mathrm{XI}-1940$, Wagenknecht s.n. (MO 1294135); Prov. Limari, $1 \mathrm{~km} \mathrm{~W}$ of road to Parque Nacional Fray Jorge, $30^{\circ} 37^{\prime} \mathrm{S}-71^{\circ} 33^{\prime} \mathrm{W}$, 200 m, 4-XI-2006, Tepe et al. 1727 (CONC); Región de Valparaíso. Prov. San Antonio, Rocas de Santo Domingo, 25 m, 20-X-1950, Pfister \& Ricardi s.n. (CONC 9760); Prov. 
Petorca, Carretera Panamericana, $3 \mathrm{~km}$ antes del puente Guaquén, 15-X-1971, C. Marticorena et al. 1307 (BAB, LP, CONC); Longotoma, 6-I-1970, Crisci 349 (LP); Papudo, above coastal cliffs, 10-XI-2006, 32 $2^{\circ} 30^{\prime} 21^{\prime \prime} \mathrm{S}, 71^{\circ} 28^{\prime} 08^{\prime} \mathrm{W}$, Tepe et al. 1923 (CONC); Prov. Valparaíso, Cerros de L. Verde, 11-II-1931, Garaventa 1965 (CONC); Valparaíso, JBN de Viña del Mar, Quebrada Los Colectivos, 21-I-2005, Novoa s.n. (CONC 162506); ca. $6 \mathrm{~km} \mathrm{~N}$ of Viña del Mar

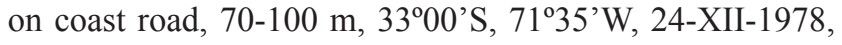
J. \& A. Solomon 4226 (MO); Quintero, 20 m, XI-1952, Hochleitner s.n. (CONC 116164); ídem, 20 m, IX-1923, Werdermann 37 (SI); Quintero, 6-II-1952, Levi Heins 154 (CONC); Quintero, Los Molles, 32 $46^{\circ} \mathrm{S}, 71^{\circ} 32^{\prime} \mathrm{W}, 20 \mathrm{~m}$, XII-1951, Gunckel 23443 (CONC); ídem, 20 m, Gunckel 40589 (CONC); Ritoque, Loma de las Piedras, 3246’S, 71³2’W, 10 m, II-1963, Gunckel 40215 (CONC). Región Metropolitana de Santiago. Prov. Santiago, San Antonio, 10 m, 16-X-1927, Montero 222 (CONC). Región del Libertador General Bernardo O'Higgins. Prov. Cardenal Caro, Tanumé, Potrero Ramírez, 28-I-2005, 34 $12^{\prime}$ 'S, $71^{\circ} 57^{\prime} \mathrm{W}$, Vera \& Ortiz 21 (CONC); Tanumé, Los Romeros, $34^{\circ} 12^{\prime} \mathrm{S}-71^{\circ} 57^{\prime} \mathrm{W}, 350$ m, 28-I-2005, Vera \& Ortiz 151 (CONC); Loma El Camino, $350 \mathrm{~m}, 30-\mathrm{I}-2005,34^{\circ} 12^{\prime} \mathrm{S}, 7^{\circ} 57^{\prime} \mathrm{W}$, Vera \& Ortiz 136 (CONC). Región del Maule. Prov. Cauquenes, Reserva Nacional Los Queules, 462 m, 35'59'16"S, 7241'35'W, 6-I-2005, A. Marticorena \& Jiménez 214 (CONC); Prov. Curicó, Llico, 15-XII-1938, Barros 2279 (LP); 8 km al N de Reserva Nacional Laguna Torca, quebrada y cerros frente a la playa, $34^{\circ} 42^{\prime} \mathrm{S}-72^{\circ} 02^{\prime} \mathrm{W}, 20 \mathrm{~m}, 13-\mathrm{XII}-2000$, Finot \& Leppe 97 (CONC). Prov. Talca, Constitución, Los Molinos, 30 m, 25-X-1958, Barnier 225 (CONC). Región del Biobío. Prov. Arauco, camino a Playa Quiala, $212 \mathrm{~m}, 37^{\circ} 20^{\prime} \mathrm{S}$, 733' 'W, 13-I-2001, Parra \& Torres 223 (CONC); 2,5 km al N de Quidico, 45 m, 38 $8^{\circ} 12^{\prime}$ S, $7^{\circ} 27^{\prime}$ W, 21-II-2001, Parra \& Torres 819 (CONC); camino a Yani, $179 \mathrm{~m}, 37^{\circ} 21^{\prime} \mathrm{S}$, 733' W, 13-I-2001, Parra \& Torres 163 (CONC); a 13 km del cruce a Ranquilco, en camino Lebu-Los Álamos, 150 m, 37 $44^{\circ}$ 'S, 73 $30^{\circ}$ 'W, 23-II-2001, Parra \& Torres 1026 (CONC); Caleta Rumena, $8 \mathrm{~m}, 37^{\circ} 10^{\prime} \mathrm{S}, 7^{\circ} 36^{\prime} \mathrm{W}, 3-\mathrm{I}-$ 2001, Baeza \& López 2759 (CONC); Prov. Biobío, camino de Biobío a Santa Bárbara, cerca del Puente Río Queuco, $450 \mathrm{~m}, 37^{\circ} 50^{\prime} \mathrm{S}, 71^{\circ} 40 \mathrm{~W}, 14-\mathrm{III}-1976$, C. Marticorena et al. 1006 (CONC); Prov. Concepción, Rocoto, La Puntilla, 30 m, 22-X-1970, Palma \& Inostroza s.n. (CONC 35172); Rocoto, 30 m, 15-XI-1967, Villarroel \& Weldt 123 (CONC); Concepción, 10 m, XII-1930, Jaffuel 1306 (CONC); Cerros de La Toma, 200 m, 30-XI-1934, Junge s.n. (CONC 5182); Estero Membrillo, cerca de Agua de la Gloria, $150 \mathrm{~m}$, 365ㄴ'S, 7254'W, 6-X-1980, R. Rodríguez 1476 (CONC); Isla Quiriquina, 20 m, 27-XI-1924, Gunckel s.n. (CONC 116257); desembocadura del Bío-Bío, 5 m, 20-XII-1950, Ricardi s.n. (CONC 10643); boca del Bío-Bío, lado Norte, 5 m, 9-XI-1935, Pfister s.n. (CONC 3557); la junta entre los ríos Bío-Bío y Mininco, 110 m, 5-XII-1953, Sparre \& Smith
80 (CONC); Caleta Burca, 36² $28^{\prime} \mathrm{S}, 72^{\circ} 54^{\prime} \mathrm{W}, 7-\mathrm{XII}-1980$, Ugarte 174 (CONC); Tomé Collen, 100 m, 24-XII-1934, Junge s.n. (CONC 5253); Lirquen, Quebrada Honda, 100 m, 8-XII-1941, Behn s.n. (CONC 20872); Prov. Ñuble, camino a Cobquecura, aproximadamente $10 \mathrm{~km}$ después del desvío hacia El Boldo, $435 \mathrm{~m}, 36^{\circ} 07^{\prime} \mathrm{S}, 7^{\circ} 38^{\prime} \mathrm{W}, 6-X I I-2001$, Mihoc et al. 3308 (CONC); Río Colmuyao interior, $35 \mathrm{~m}$, $36^{\circ} 17^{\prime}$ 'S, $72^{\circ} 47^{\prime} \mathrm{W}$, XII-2001, Mihoc \& Teneb 3897 (CONC). Región de La Araucanía. Prov. Cautín, Temuco, 130 m, XI1946, Gunckel 43572 (CONC); Cunco (Huichahue), $200 \mathrm{~m}$, 15-XII-1930, Aravena 271 (CONC); Toltén, 10 m, 21-III1942, Gunckel 12826 (CONC); Puente Loncotripai, a 15 km de Tirúa, 120 m, $38^{\circ} 24^{\prime} \mathrm{S}, 73^{\circ} 23^{\prime} \mathrm{W}, 21-\mathrm{II}-2001$, Parra \& Torres 782 (CONC). Región de Los Lagos. Prov. Chiloé, Isla Grande de Chiloé, trayecto Piruquina a Alcaldeo de LlauLlao, cerca del Río Chilcán, 175 m, 42²3'S, 7352'W, 14 II-1984, Villagrán 5699 (CONC); Isla Quilán, entre Punta

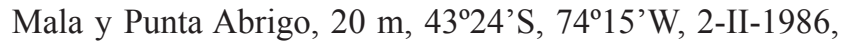
Villagrán \& Leiva 7269 (CONC); Isla Guafo, trayecto desde Caleta Samuel al Faro, 100 m, 4336'S, 74²4 'W, 25-II1986, Villagrán \& Leiva 7545 (CONC); Prov. Llanquihue, Petrohué, 210 m, 14-I-1946, Pfister s.n. (CONC 6012); Lago Llanquihue, Ensenada, 60 m, 20-II-1944, Pfister 786, 4278 (CONC); ídem, La Poza, 100 m, 8-IV-1939, Looser 66209 (CONC); Prov. Osorno, Lliflén, Cerro Lliflén, 125 m, 23-II1958, C. Marticorena \& Furet 62 (CONC); Lago Puyehue, Isla Fresia, 250 m, I-II-1956, Levi Heins 2911 (CONC); Prov. Palena, Hornopirén, $40 \mathrm{~m}, 41^{\circ} 58^{\prime} \mathrm{S}, 7^{\circ} 25^{\prime} \mathrm{W}, 2-\mathrm{I}-$ 2002, Aedo 7351 (CONC). Región de Los Ríos. Prov. Valdivia, Cerro Esmeralda, $120 \mathrm{~m}, 40^{\circ} 18^{\prime} \mathrm{S}, 7^{\circ} 27^{\prime} \mathrm{W}$, II1958, Schlegel 1537 (CONC); Valdivia, Cno. Costanera, 10 m, 10-IV-1973, Montero 9005 (CONC); Playa de Mehuin, 20 m, 11-III-1964, Santos 154 (CONC); Mehuin, $20 \mathrm{~m}$, 18-II-1976, Montero 10151 (CONC); Corral, 25 m, 28XI-1932, Gunckel 3324 (CONC); ídem, 25 m, 30-I-1933, Gunckel s.n. (CONC 46204); Corral, Niebla, 25 m, 11-I1964, Gunckel 41862 (CONC); San Juan, 50 m, 8-II-1930, Gunckel 1488 (CONC); Morro Gonzalo, 160 m, 23-I-1932, Gunckel 2999 (CONC); ídem, 50 m, 6-XI-1936, Gunckel 19193 (CONC); Quebrada La Aguada, 25 m, 22-II-1932, Gunckel 3196 (CONC); ídem, 50 m, 10-III-1936, Gunckel 28012 (CONC); San Carlos, 50 m, 10-X-1931, Gunckel 36408 (CONC). Dpto. La Unión, camino entre Hueicolla y La Unión, km 2, 22-I-1971, Weldt-Rodríguez 946 (CONC); Villarrica, Welturne, cataratas de Huilo-Huilo, $520 \mathrm{~m}, 27-$ III-1980, Montero 11575 (CONC). Archipiélago Juan Fernández, Isla Masatierra, 400 m, II-1965, Fuhrmann s.n. (CONC 116172); ídem, $30 \mathrm{~m}, 33^{\circ} 38^{\prime} \mathrm{S}, 78^{\circ} 50^{\prime} \mathrm{W}, 10-\mathrm{XI}-$ 1966, Angulo 105 (CONC); ídem, Quebrada de El Inglés, 400 m, 14-XII-1965, Muñoz \& Sierra 7327 (CONC); ́́dem, Punto San Carlos, Cumberland Bay, 26-XII-1965, Meyer 9684 (CONC); ídem, V. Colonial, 450 m, 25-II-1955, Sparre 121 (CONC); ídem, San Juan Bautista, 50 m, 333's, 7850'W, 13-I-1996, Swenson \& Stuessy 399 (CONC); 
ídem, between El Castillo and the electric generating plant for the town, 7-II-1990, Stuessy \& López 11449 (CONC); ídem, La Vaquería, 20 m, 26-I-1984, Stuessy \& Crawford 6436 (CONC); ídem, 10-100 m, 5-II-1990, López 11403 (CONC); ídem, in open areas along sea cliffs, 90 m, 15-I1984, Stuessy et al. 6201 (CONC).

4. Pseudognaphalium cymatoides (Kunze ex DC.) Anderb., Opera Bot. 104: 147. 1991. Gnaphalium cymatoides Kunze ex DC., Prodr. 6: 225. 1838. TYPE: Chile. Andes, Concon, 4200 m, Dec., E. F. Poeppig s.n., Diar. 379 (lectotype, here designated, P 00704542, = photo!; isolectotype, HAL $0111785,=$ photo!). Fig. 8.

Gnaphalium ulophyllum Hook. \& Arn., Bot. Beechey Voy. 31. 1841, syn. nov. TYPE: Chile. Valparaíso, Playa Ancha 1832, T. C. Bridges 229 (lectotype, here designated, K 000500321, = photo!; isolectotype, E 00369125, = photo!)

Gnaphalium cymatoides Kunze ex DC. var. glabrum Walp., Nov. Actorum Acad. Caes. Leop.-Carol. Nat. Cur. 19 (Suppl. 1): 276. 1843. TYPE: 'Chile. Valparaiso' (not seen).

Gnaphalium moelleri Phil., Anales Univ. Chile 90: 11. 1895. Pseudognaphalium moelleri (Phil.) Anderb., Opera Bot. 104: 147. 1991, syn. nov. TYPE: Chile. Biobío, Renaico, III-1885, R.A. Philippi s.n. (lectotype, here designated, SGO 64417!); isolectotypes K 000500403, =photo!, B (probably destroyed), =photo 15109 !

Gnaphalium canum Phil., Anales Univ. Chile 90: 11. 1895, nom. illeg., non Wallich, 1831, syn. nov. TYPE: Chile. Biobío, Renaico, III-1887, Philippi s.n. (lectotype, here designated, SGO 64418!; isolectotypes, LP 001902!, LP 001903!, SGO 44965!).

Annual or biennial herbs, (7)10-40(50) cm tall, multistemmed (rarely single stem), stems erect or ascending, branched, weak, glandular-arachnoid. Leaves 9-35 × 1-2(6) mm, linear, margin revolute, conspicuously undulate, apex acute, mucronate, base broad, scarcely decurrent; concolorous, arachnoid-glandular on both surfaces, trichomes of 2 types, short glandular biseriate and few long eglandular eseptate, 3-4-celular trichomes. Capitula in clusters arranged in lax corymbs or panicles. Involucre campanulate, 2-3 $\times 2-3 \mathrm{~mm}$. Phyllaries 3-seriate, lamina whitish or yellowish, hyaline and shiny, apex obtuse; outer phyllaries $2-3 \times 0.8-1.5 \mathrm{~mm}$, ovate; inner phyllaries 2.5-3 $\times$ 0.4-1 mm, oblong-obovate. Pistillate florets 40-95(130), corolla yellow, filiform, 1.5-3 mm. Bisexual florets 4-8(11-12), corolla yellow, tubulose, $1.8-3 \mathrm{~mm}$ long. Achenes $0.3-1 \mathrm{~mm}$ long, pilose with short oblong duplex hairs. Pappus bristles 1.5-2.2 mm long.
Phenology. Flowering from (July, August) September to May.

Distribution And habitat. Bolivia (Hind 2011), Argentina (Chubut, Neuquén, Río Negro) and North, Central and South Chile (Arica y Parinacota, Coquimbo, Valparaíso, Metropolitana, Libertador General Bernardo O'Higgins, Maule, Biobío, Araucanía, Los Lagos), growing from sea level to $3600 \mathrm{~m}$.

Relationships. Pseudognaphalium cymatoides is closely related to $P$. gayanum. Both species have undulate leaf blade margins. Pseudognaphalium cymatoides can be distinguished by its weakly branched stems and involucres 2-3 mm high (vs. robust unbranched stems and involucres 4-5 $\mathrm{mm}$ high in P. gayanum).

\section{OBSERVATIONS}

1. Candolle (1838: 225) mentioned two syntypes in the protologue of Gnaphalium cymatoides: 'In Chili circa Concon (Poepp.!) in lapidosis maritimis secus torrentes ad Valparaiso et S. Iago (Bert.!)'. We found these two collections kept at P and MPU, Andes, 4200' Decbr. et ad Concon, (Diar. 379!!), Poeppig s.n., P 704542, and Valparaiso: Prope St. Yago, ad follas et lepos, Bertero s.n in $3 / 1829$, MPU 16284, respectively. We propose the material 'Concon, Poeppig s.n.', which presents the most complete plant, as lectotype of Gnaphalium cymatoides.

2. We propose to place Gnaphalium ulophyllum (which is characterized in the protologue by its linear leaves arachnoid pubescent with undulate margins) into synonymy of $P$. cymatoides because there is no diagnostic character that differentiates the former from the latter.

3. According to the protologue, Gnaphalium ulophyllum Hook. \& Arn., was based on specimen 'Valparaiso, Mr. Bridges'. We located two sheets at E and K: E 00369125 , $\mathrm{K} 000500321$. We selected the collection 'K 000500321', which is better preserved.

4. Philippi (1895: 11) mentioned two syntypes in the protologue of Gnaphalium moelleri, 'In arvis inter Renaico et Negrete in provincia Biobío'. We found these two collections kept at SGO, [Negrete, III 85], SGO 44977 and [Renaico, Martio 1885], SGO 64417. We propose the material SGO 64417, which presents the most complete plant, as lectotype of Gnaphalium moelleri.

5. Gnaphalium moelleri Phil. is placed into synonymy of $G$. cymatoides Kuntze ex DC. because there is no diagnostic character that differentiates the former from the latter. Both are glandular-arachnoid plants with linear leaves broad at base with undulate margins and capitula in clusters arranged in lax corymbs or panicles. 


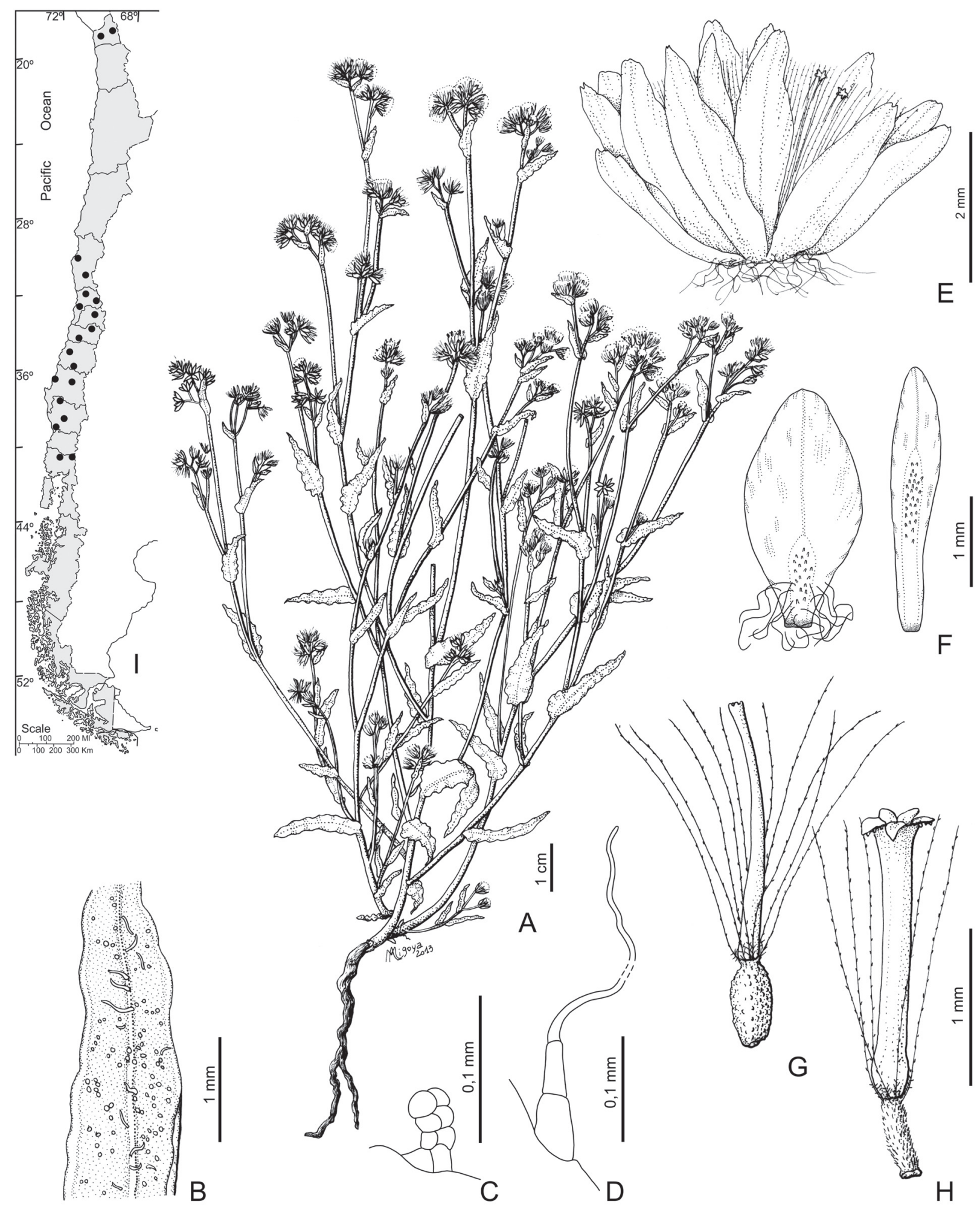

Figure 8. Pseudognaphalium cymatoides. A, Habit; B, Leaf pubescence; C, D, Foliar trichomes; E, Capitulum; F, Phyllaries; G, Pistillate floret; H, Bisexual floret. I, Distribution [A-H, Gay s.n., GH].

Figura 8. Pseudognaphalium cymatoides. A, Planta; B, Pubescencia de la hoja; C, D, Tricomas foliares; E, Capítulo; F, Filarios; G, Flor pistilada; H, Flor perfecta. I, Distribución [A-H, Gay s.n., GH]. 
6. According to the protologue, Gnaphalium canum Phil., was based on specimen 'Renaico, III-1887'. We located four sheets at LP and SGO: LP 001902, 001903; SGO 64418, 44965. We selected as lectotype the collection 'SGO 64418' which is better preserved and has Cabrera's annotation 'Typus'.

7. Gnaphalium canum Phil. which was previously placed into synonymy of G. moelleri Phil. (Cabrera 1971) is here considered as synonym of Pseudognaphalium cymatoides.

Additional material examined. CHILE. Región de Arica y Parinacota. Prov. Arica, Quebrada de Ipilla, 3000 m, $18^{\circ} 37^{\prime} \mathrm{S}, 6^{\circ} 28^{\prime} \mathrm{W}, 11-\mathrm{IX}-1963$, Schlegel 4895 (CONC); Prov. Parinacota, cerros frente a Putre, $3600 \mathrm{~m}, 18^{\circ} 12^{\prime} \mathrm{S}$, 69³4'W, 21-III-1987, Matthei \& Rodríguez 269 (CONC). Sin loc. determinada, 1838-1842, Herbarium of the U.S. South Pacific Exploring Expedition under the command of Capt. Wilkes, U.S.N (PH 01084668, GH 00282516); ídem, Cuming 334 (SI). Región de Coquimbo. Prov. Choapa, Illapel, 20-I-1942, Barros 2260 (SI); ídem, 1838-1842, Philippi s.n. (NY); Illapel, Carretera Panamericana, 15 km N de Quilimarí, ca. Totoralillo, 25 m, 15-X-1971, C. Marticorena et al. 1338 (CONC); Illapel, Caleta Oscuro, 5-50 m, 31 ${ }^{\circ} 25^{\prime}$ 'S, $71^{\circ} 35^{\prime} \mathrm{W}, 2-\mathrm{XI}-1974$, C. Marticorena et al. 276 (CONC); $2,1 \mathrm{~km} \mathrm{~N}$ of Puerto Oscuro on the Panamericana, $200 \mathrm{~m}, 31^{\circ} 24^{\prime} \mathrm{S}, 7^{\circ} 35^{\prime} \mathrm{W}, 18-\mathrm{IX}-1994$, Bliss \& Lusk 575 (CONC); ídem, 20-XI-1994, Bliss 674 (CONC); Caleta Manso, 9 km N de Huentelauquén, 20 m, 31 3030'S, $71^{\circ} 34^{\prime} \mathrm{W}$, 31-X-2000, Teillier \& Márquez 4870 (CONC); Prov. Elqui, Coquimbo, VII- VIII 1856, Harvey s.n. (GH 00282514); 16 $\mathrm{km} \mathrm{N}$ of 'Camping Morillos' (84 km N of Los Socos) on rte. 5, 100 m, 11-II-1988, C. Marticorena et al. 9972 (CONC); Carretera Panamericana entre Socos y Coquimbo, km 84, Alto del Centinela, $100 \mathrm{~m}, 30^{\circ} 01^{\prime} \mathrm{S}, 7^{\circ} 23^{\prime} \mathrm{W}, 11-\mathrm{II}-1988$, C. Marticorena et al. 9972 (CONC); Andacollo, 11-X-1958, Ricardi \& C. Marticorena 4943 (CONC); ídem, Ricardi \& C. Marticorena 4943 bis (CONC); Prov. Limarí, Ovalle, Tulahuén, 15-I-1942, Barros 2255 (SI); Ovalle, Talinay, $700 \mathrm{~m}, 30^{\circ} 50^{\prime} \mathrm{S}, 7^{\circ} 37^{\prime} \mathrm{W}$, 4-III-1950, Jiles 1686 (CONC); Ovalle, Río Limarí, 180 m, 30³7’S, 71²12’W, 16-XII-1948, Jiles 1112 (CONC). Región de Valparaíso. Prov. Los Andes, Portillo, Laguna del Inca, 3200 m, 32²50'S, 7009'W, 11-III1994, Teillier \& Niemeyer 3322 (CONC); Prov. Quillota, La Cruz, Fundo Sta. Ana, 100 m, 22-IV-1945, Behn s.n. (CONC 20871); Prov. Petorca, Zapallar, 35 m, 15-XI-1928, Behn s.n. (CONC 20870); Zapallar, Cerro Francés, 600 m, 23-II-1953, Levi Heins 150 (CONC); ídem, 20-II-1953, Levi Heins 174 (CONC); Prov. San Antonio, Palos Quemados, 22-I-1933, Looser 66226 (MO); Prov. San Felipe de Aconcagua, Jahuel, El Zaino, 1100 m, 11-XI-1955, Barrientos 1699 (CONC); Prov. Valparaíso,Viña del Mar, 21-II-1939, Burkart 9383 (SI); Quintero, 40 m, XII-1950, Gunckel 21612 (CONC);
Quintero, Los Juanes, 20 m, IX-1953, Navas s.n. (CONC 116177 y 116178); ídem, 20-I-1954, Navas s.n. (CONC 116326); Quintero, Dunas de Ritoque, $10 \mathrm{~m}, 32^{\circ} 46^{\prime} \mathrm{S}$, $71^{\circ} 32^{\prime}$ W, II-1963, Gunckel 40170 (CONC); Prov. MargaMarga, Valle de Marga-Marga, IX-1932, Jaffuel \& Pirion 3026; ídem, 1930-32, Jaffuel \& Pirion 3226 (CONC); Marga-Marga, 150 m, I-1914, Jaffuel s.n. (COvillagránNC 89741); S/Prov., Laguna Peñuelas, 350 m, 27-II-1952, s/leg. (CONC 13243); Tranque Orosco, 290 m, V-1966, Zöllner 1068 (CONC). Región Metropolitana de Santiago. Prov. Santiago, Santiago, s .f., M. Cl. Gay s.n. (GH); Quebrada de La Plata, 740 m, 33²9'S, 7054'W, 23-XI-1960, Schlegel 3199 (CONC); ídem, laderas de los cerros Morro El Fraile y Buitreras, 540-630 m, 33⒉ $9^{\prime} \mathrm{S}, 70^{\circ} 50^{\prime} \mathrm{W}, 4 / 18-\mathrm{II}-2001$, Mihoc 4 (CONC); Santuario de la Naturaleza Yerba Loca, vega en ladera al SW del estero Yerba Loca, $1880 \mathrm{~m}, 33^{\circ} 20^{\prime} \mathrm{S}$, 70¹9’W, 31-I-1999, Arroyo \& Humaña 99887 (CONC); El Arrayán, 14-I-1954, Arriagada s.n. (CONC 116277); Cerro San Cristóbal, 800 m, 14-V-1954, Navas 632 (CONC). Región del Libertador General Bernardo O'Higgins. Prov. Cachapoal, Rancagua, 2-I-1938, Barros 2307 (SI); Prov. Colchagua, Río Claro, La Rufina, 315 m, I-II-1959, Barrientos 2025 (CONC); S/Prov., Las Peñas, 755 m, 25I-1958, Barrientos 1621 (CONC). Región del Maule. Prov. Linares, Camino Parral-Cauquenes, km 10, Parcela 2, El Cairo, 150 m, 360ㅜ' $, 71^{\circ} 54^{\prime} \mathrm{W}, 17-\mathrm{III}-1988$, Rodríguez \& Baeza 2371 (CONC); Prov. Talca, Los Bellotos del Melado,

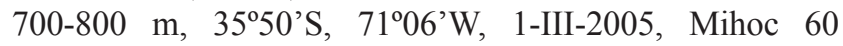
(CONC). Región del Biobío. Prov. Biobío, Negrete, a orillas del Bío-Bío, 75 m, 19-II-1951, Pfister s.n. (CONC 10503); Prov. Concepción, Hualpén, 20 m, 9-III-1940, Junge s.n. (CONC 6771); Prov. Nuble, Quirihue, 200 m, 12-IV-1952, Zemelman s.n. (CONC 13277); camino a Recinto, $950 \mathrm{~m}$, 26-IV-1954, Ledezma 8 (CONC). Región de La Araucanía. Prov. Cautín, Temuco, ca. Río Cautín, 110 m, 14-II-1941, Gunckel 11011 (CONC); Temuco, Truf Truf, 125 m, 20-XII1960, Montero 6364 (CONC); Almagro, 50 m, 21-II-1943, Gunckel s.n. (CONC 116238); Cajón, 130 m, 26-III-1946, Cañulaf s.n. (CONC 116241); Toltén, lado N desembocadura Río Toltén, 10 m, 21-III-1942, Gunckel 12827 (CONC); Volcán Villa-Rica, 1300 m, I-1926, Hollermayer s.n. (CONC 116282); Prov. Malleco, Angol, peddly gravelly shore of Río Malleco, 100-150 m, 27-28-II-1925, Pennell 12845 (PH). Región de Los Lagos. Prov. Osorno, Ribera N del Lago Rupanco, Fundo Pichilafquen, 120 m, 13-II-1955, Pfister s.n. (CONC 18562); Pichilafquén-Lago Rupanco, $120 \mathrm{~m}$, 15-II-1956, Pfister s.n. (CONC 19025).

5. Pseudognaphalium gayanum (J. Rémy) Anderb., Opera Bot. 104: 147. 1991. Gnaphalium gayanum J. Rémy in Gay, Fl. Chilena 4(3): 225. 1849. TYPE: Chile. Colchagua, Cordillera de San Fernando, Talcaregue, II-1831, C. Gay s.n. (holotype, P 00704552, = photo!). Fig. 9. 

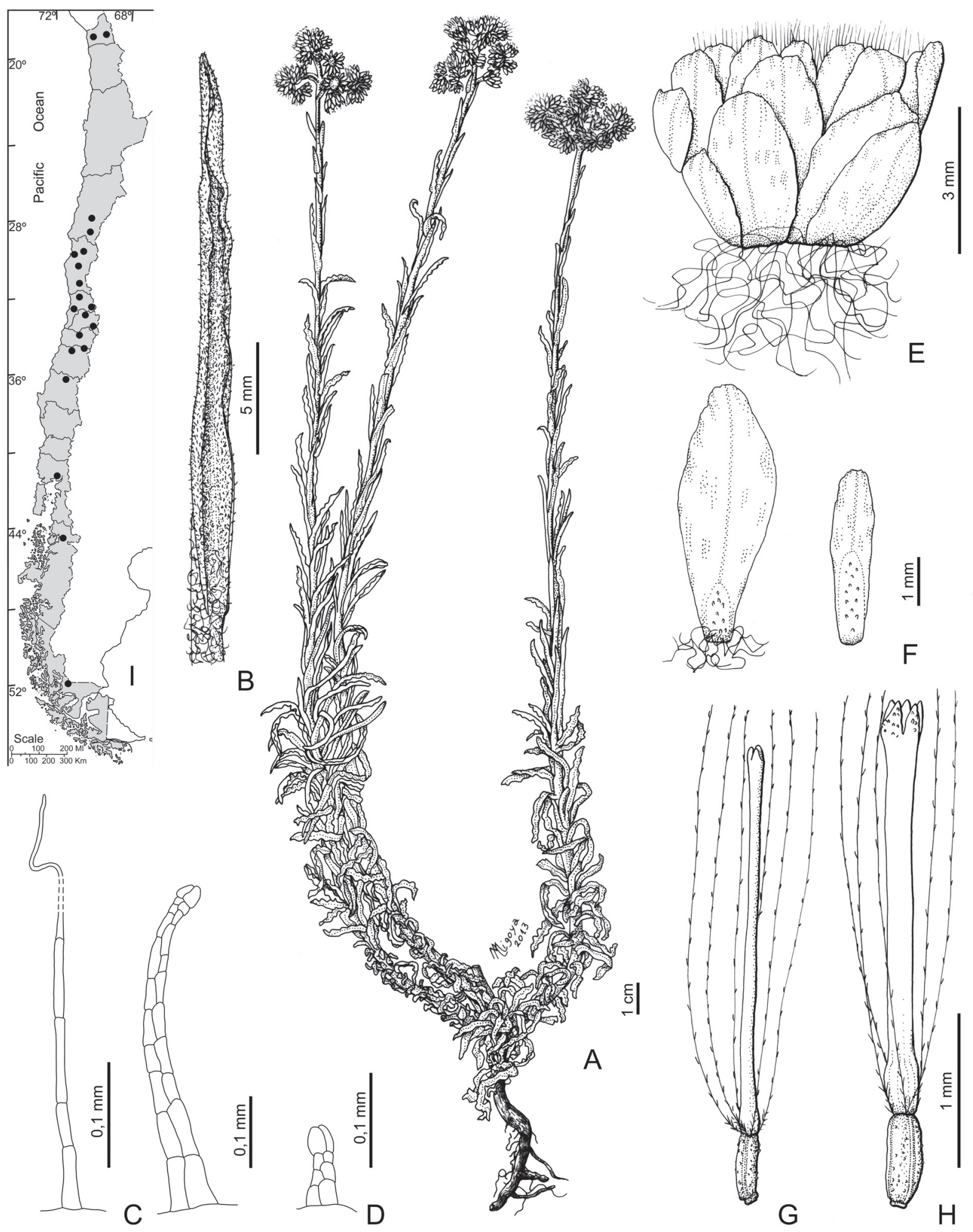

FiguRE 9. Pseudognaphalium gayanum. A, Habit; B, Leaf pubescence; C, D, Foliar trichomes; E, Capitulum; F, Phyllaries; G, Pistillate floret; H, Bisexual floret. I, Distribution [A-H, Werdermann 470, NY].

Figura 9. Pseudognaphalium gayanum. A, Planta; B, Pubescencia de la hoja; C, D, Tricomas foliares; E, Capítulo; F, Filarios; G, Flor pistilada; H, Flor perfecta; I, Distribución [A-H, Werdermann 470, NY]. 
Gnaphalium heterotrichum Phil., Linnaea 29: 4. 1857-58. Pseudognaphalium heterotrichum (Phil.) Anderb., Opera Bot. 104: 147. 1991, syn. nov. TYPE: Chile. Coquimbo, La Serena, X-1837, C. Gay 681 (holotype, SGO 71285!; isotype, B (probably destroyed), = photo F15100 SI!),

Gnaphalium leucocephalum Phil., Linnaea 33: 166. 1864, nom. illeg., non A. Gray, 1853. Gnaphalium pseudohelichrysum Reiche, Anales Univ. Chile 112: 112. 1903, nov. nom., syn. nov. TYPE: Chile. Colchagua, 2-3000 m s.m., XII-1860, Landbeck s.n. (holotype, SGO 64445!; isotype, B probably destroyed).

Gnaphalium heterophyllum Phil., Anales Univ. Chile 90: 14. 1895, syn. nov. TYPE: Chile. Coquimbo, year 1888 , W. Geisze s.n. (lectotype, here designated, SGO 64403!; isolectotypes, LP 001921!, SGO 64402!, 64404!, 72256!)

Gnaphalium robustum Phil., Anales Univ. Chile 90: 16. 1895. Pseudognaphalium robustum (Phil) Anderb., Opera Bot. 104: 148. 1991, syn. nov. TYPE: Chile. Santiago, Rio Colorado, I- 1888, Philippi s.n. (holotype, SGO 64424!).

Perennial herbs, $30-110 \mathrm{~cm}$ tall, multistemmed, stems erect or ascending, unbranched, robust, arachnoid-glandular to whitish-woolly. Leaves (30)40-60(90) $\times 2-4(10)$ $\mathrm{mm}$, linear, margins conspicuously undulate, apex acute, mucronate, base short-decurrent 5-11 mm long, sometimes broad; concolorous, glandular-arachnoid, trichomes of 2 types on both surfaces, glandular biseriate and few long eglandular eseptate, 5-celular trichomes. Capitula in clusters arranged in corymbs or panicles (seen in large plants). Involucre broadly campanulate $4-5 \times 4-5 \mathrm{~mm}$. Phyllaries 4-seriate, lamina light-brown, yellowish, hyaline and shiny or opaque white, apex obtuse to rounded; outer phyllaries ca. $5 \times 2 \mathrm{~mm}$, ovate; inner phyllaries ca. $3.5 \times 1 \mathrm{~mm}$, oblongobovate. Pistillate florets 84-181, corolla yellow, filiform, 2-2.6(3) mm long. Bisexual florets (7)13-17(31), corolla yellow, tubular, $2-3.6 \mathrm{~mm}$ long. Achenes $0.1-0.3 \mathrm{~mm}$ long, pilose with short oblong duplex hairs. Pappus bristles 2.2$3.4 \mathrm{~mm}$ long.

Phenology. Flowering from (May, July) September to April.

Vernacular name. 'Té de burro'.

Distribution and habitat. Endemic to Chile from northern, central and southern Regions (Arica y Parinacota, Atacama, Coquimbo, Valparaíso, Metropolitana, Libertador General Bernardo O'Higgins, Maule, Biobío, Los Lagos, Aysén del General Carlos Ibañez del Campo, Magallanes y de la Antártica), growing from sea level to $3500 \mathrm{~m}$.

Relationships. Pseudognaphalium gayanum is closely related to P. cymatoides. Both species have conspicuously undulate leaf blade margins. Pseudognaphalium gayanum can be distinguished by its robust unbranched stems and involucres $4-5 \mathrm{~mm}$ high (vs. weakly branched stems and involucres 3-4 $\mathrm{mm}$ high in $P$. cymatoides).

\section{OBSERVATIONS}

1. Muñoz Pizarro (1960: 142) cited as holotype of Gnaphalium heterotrichum the collection 'cerca de La Serena, Gay. Herb, Chil. No 681, 64406'. However at SGO was located the collection 'Prov. Coquimbo, Serena, 8bre. 1837, [Gay] 681, 71285'. Probably '64406' instead of ' $71285^{\prime}$ ' was a transcription error.

2. Gnaphalium heterotrichum Phil. was described on the basis of its white tomentose stems. However, this is a variable feature throughout the range of Gnaphalium gayanum J. Rémy.

3. Gnaphalium leucocephalum Phil.(=G.pseudohelichrysum Reiche, nov. nom.) was described on the basis of its white-yellowish phyllaries and white tomentose leaves. However, these are variable features throughout the range of Gnaphalium gayanum J. Rémy.

4. There is a photo-Field Museum Nr.15107 ex B, with a specimen collected by Philippi in Cordillera de Linares. Since the protologue of Gnaphalium leucocephalum mentions 'Landbeck, Colchagua' this specimen is excluded from the type collection of G. leucocephalum.

5. According to the protologue, Gnaphalium heterophyllum Phil. was based on the specimen 'Frequens in provincia Coquimbo'. We located four sheets at SGO: 'Coquimbo', 'Coquimbo, 10. 1878', 'Coquimbo, sept. 1888' and 'Coquimbo, W. Geisze, 1888'. We selected as lectotype the collection 'Coquimbo, W. Geisze, 1888' which is better preserved.

6. Gnaphalium heterophyllum Phil. which was previously placed into synonymy of $G$. heterotrichum Phil. (Freire \& Iharlegui 2008) is here considered as synonym of Pseudognaphalium gayanum.

7. After examining the type material, we propose to place Gnaphalium robustum Phil. into synonymy of G. gayanum J. Rémy, because there is no character that differentiates the former from the latter. Both are multistemmed, arachnoidglandular to whitish-woolly plants with unbranched woody stems, and linear leaves, undulate on margins.

Additional material examined. CHILE. Región de Arica y Parinacota. Prov. Arica, camino de Arica a Portezuelo de Chapiquiña km 96, 3450 m, 9-II-1964, C. Marticorena et al. 
46 (CONC); Prov. Parinacota, Camino a Putre, $3200 \mathrm{~m}$, $18^{\circ} 12^{\prime} \mathrm{S}, 6^{\circ} 34^{\prime} \mathrm{W}, 11-\mathrm{V}-2000$, Belmonte 20154 (CONC); camino Zapahuira a Putre, km 13, 3300 m, 5-V-1972, Ricardi et al. 155 (CONC). S/Región, Río Blanco, 2000 m, 27-XII1964, Zöllner 1076 (LP). S/loc., 13-VI-1948, Bultmann 23661 (CONC); s/loc., s/fecha, Hno. C. Joseph 3657 (CONC). Región de Atacama. Prov. Copiapó, Totoral, ca. 100 m, II-1924, E. Werdermann 470 (LP, MO, NY, SI); Prov. Huasco, $1 \mathrm{~km} \mathrm{~S}$ of Huasco, 20-X-1955, Böcher et al. 543 (LP); Quebrada del Pretil, 14-XI-1956, Ricardi \& C. Marticorena 3956 (CONC); Carrizal Bajo, Mina Oriente,

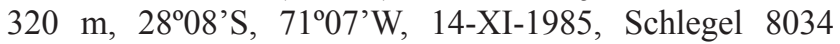
(CONC). Región de Coquimbo. Prov. Choapa, Illapel, s. f., Philippi s.n. (NY); ídem, 310 m, 11-IX-1924, Barros 3404 (CONC); ídem, 310 m, IX-1926, Barros 1467 (CONC); Illapel, Cuesta del Espino, 3-X-1941, Barros 2318 (LP); Caren, near Quebrada Luncuman, ca. $40 \mathrm{~km}$ from Illapel, tributary Illapel river., ca. $1000 \mathrm{~m}, 14-\mathrm{XI}-1938$, Worth \& Morrison 16494 (MO, SI); Caleta Manso, $9 \mathrm{~km} \mathrm{~N}$ Huentelauquén, 20 m, 31 30'S, 71 $34^{\circ} \mathrm{W}, 31-\mathrm{X}-2000$, Teillier \& Márquez 4848 (CONC); Valle Ojotas, cordillera Quelen, cerca de Cuncumen, 2500 m, I-1984, Zöllner 11790 (SI); Prov. Elqui, Cuesta Buenos Aires nördl La Serena, $29^{\circ}$ 34'S, 71 15'W, 500 m, 11-XII, 1987, Rosas 1385 (M);

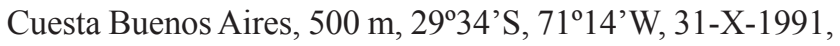
Rodríguez 2789 (CONC); Cuesta de Buenos Aires, cerca del Portezuelo, 550 m, 20-X-1971, C. Marticorena et al. 1612 (CONC); entre Coquimbo y Buyarán, 100 m, 8-XI-1923, Werdermann 107 (SI); Coquimbo, de La Serena a Ovalle, 41 $\mathrm{km}$ sur de La Serena, cuesta las Cardas, $30^{\circ} 15^{\prime} \mathrm{S}, 71^{\circ} 17^{\prime} \mathrm{W}$, $450 \mathrm{~m}, 14-\mathrm{XII}-1987$, Rosas 1507 (M); Coquimbo 30 ${ }^{\circ} \mathrm{S}, 20-$ 30 m, 13-10-1898, Von Bayern 388 (M); Coquimbo, Playa, 15 m, 26-IX-1934, Montero 1881 (CONC); Punta de Coquimbo, 20-IX-1957, Ricardi \& C. Marticorena 4488 (CONC); ídem, 20-IX-1957, Cabrera 12697 (LP); vicinity of Coquimbo, IX-1931, Jaffuel 2681 (GH); Quebrada al Norte del Punto Juan Soldado, $29^{\circ} 39^{\prime} \mathrm{S}, 71^{\circ} 18^{\prime} \mathrm{W}, 11-\mathrm{XII}-$ 1987, Rosas 1392 (NY); Quebrada El Pangue al N de Cochiguaz y El Colorado $30^{\circ} 07^{\prime} \mathrm{S}, 70^{\circ} 23 \mathrm{~W}, 2000 \mathrm{~m}, 13-$ XII-1897, Rosas 1465 (M); Andacollo, 1000 m, 11-X-1958, Ricardi \& C. Marticorena 4972 (CONC, LP); Paihuano, III1946, Roesner s.n. (CONC 6494); Paihuano, cerca del río, 950 m, 25-IX-1948, Behn s.n. (CONC 8414 y 20882); Chanchoquí-Paihuano, 900 m, 20-IX-1948, Pfister s.n. (CONC 8363); Along the Panamerican Highway, $20 \mathrm{~km} \mathrm{~S}$ of Las Herraduras, $400 \mathrm{~m}, 30^{\circ} 05^{\prime} \mathrm{S}, 7^{\circ} 25^{\prime} \mathrm{W}, 27-\mathrm{I}-1991$, De Vore 1545 (CONC); Camino de Playa Temblador a Cruz Grande, 198 m, 29 $28^{\circ}$ 'S, $71^{\circ} 17^{\prime} \mathrm{W}, 17-I X-1989$, Niemeyer $e t$ al. 89122 (CONC); Cochiguaz, 26-V-1990, Von Bohlen 742 (CONC); La Serena, IX-1928, Barros 24725 (CONC); Punta Teatinos, $10 \mathrm{~km} \mathrm{~N}$ de La Serena, 15-X-1940, Looser 4394 (LP); puente Las Trancas, en el camino de Vicuña a Hurtado, 16-XII-1940, Wagenknecht 4396 (LP); Rivadavia, cauce del Río Turbio, 17-I-1936, Cabrera 3506 (LP); Prov. Limarí,
Altos de Talinay, 700 m, 18-XI-1978, Zöllner 10442 (2 ejemplares, MO, CONC); Talinay, $700 \mathrm{~m}, 30^{\circ} 50^{\prime} \mathrm{S}, 71^{\circ} 37^{\prime} \mathrm{W}$, 8-II-1948, Jiles 542-a (CONC); Cerro Talinay, 550 m, 8-XII1953, Kausel 3806 (LP); Cordillera de Ovalle, Río Tascadero, El Polvo, 31 ${ }^{\circ} 08^{\prime} \mathrm{S}, 7^{\circ} 35^{\prime} \mathrm{W}, 2400 \mathrm{~m}$, Jiles 6437 (M); Ovalle, Fray Jorge, X-1951, Jiles s.n. (CONC 89696); Cordillera de Ovalle, Quebrada Larga, 3000 m, 3044'S, 70²3'W, 3000 m, 17-II-1958, Jiles 3429 (CONC); Hacienda Tamaya, 250 m, 30³4'S, 71²4'W, 30-X-1956, Jiles 3047 (CONC); Estancia El Parral, 600 m, 21-IX-1950, Jiles 1848 (CONC, LP); Camino de La Serena a Ovalle por Las Cardas, km 61, pasando la Cuesta Las Cardas, Quebrada del Ingenio, 3,5 km $\mathrm{S}$ de Estación Higueritas, $380 \mathrm{~m}, 30^{\circ} 26^{\prime} \mathrm{S}, 71^{\circ} 10^{\prime} \mathrm{W}, 11-\mathrm{II}-$ 1988, C. Marticorena et al. 9956 (CONC); Quebrada del Teniente, 30 m, 9-II-1963, Ricardi et al. 790 (CONC); Carretera Panamericana, $11 \mathrm{~km} \mathrm{~S}$ de la Quebrada Teniente, 9-II-1963, Ricardi et al. 796 (CONC); Along the Panamerican Highway, $1 \mathrm{~km}$ N of Mantos de Hornillo, 250 m, 17-I-1989, Lammers et al. 6381 (CONC); Parque Nacional Fray Jorge, $500 \mathrm{~m}, 30^{\circ} 40^{\prime}$ 'S, 71²40'W, 5-XI-1974, C. Marticorena et al. 468 (CONC); ídem, 450 m, 15-IX-1947, Ibáñez et al. s.n. (CONC 144832); ídem, X-1951, Jiles s.n. (CONC 89696); ídem, 17-VII-1967, Zöllner 1772 (LP); S/Loc., 9-IX-1926, Barros 40 (LP); S/loc., 3-X-1953, Cabrera 11415 (LP). Región de Valparaíso. Prov. Los Andes, Uspallata-Pass der chilenischen Hochcordillere ( $33^{\circ} \mathrm{S}$ Breite): Juncal; zwischen Geröll, 2200 m, 20-I-1903, Buchtien s.n. (SI 022607); Juncal, 2200 m, II-1903, Buchtien 3 (MO); Cajón del Río Colorado, $3000 \mathrm{~m}, 32^{\circ} 34^{\prime} \mathrm{S}, 70^{\circ} 20^{\prime} \mathrm{W}, 20-\mathrm{II}-2002$, Teillier 5111 (CONC); Saladillo, Laguna Turquesa, Río Estero

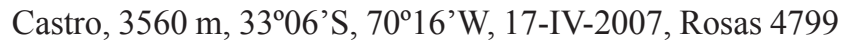
(CONC); Portillo, 2800 m, I-1970, Crisci 490 (LP); Prov. Marga Marga, Limache, 90 m, I-1948, Levi Heins 445 (CONC); Estero de Maitenes, Maitenes, Limache, 27-IX1928, Garaventa 320 (CONC); Cordillera de la Costa, cerca de Limache, en un cerro, Punta Imán, 1800 m, XI 1964, Zöllner 978 (LP); Cerro La Campana, 1200 m, 17-XII-1973, Zöllner 8088 (CONC, MO); Cerro de La Campana mirando a Olmué, 500-1000 m, 22-XI-1936, Looser 3765 (CONC); Cerro Campana, cerca de la mina 'La Pronosticada', 1300 m,19-I-1936, Garaventa 3117 (CONC); cerca de Quilpué, XI-1967, Zöllner 2200 (LP); Prov. Petorca, Rt. 5 between tunnel and La Ligua, $110 \mathrm{~m}, 32^{\circ} 33^{\prime} 25^{\prime}$ 'S, $71^{\circ} 15^{\prime} 54^{\prime \prime} \mathrm{W}$, 3-XI-2006, Tepe et al. 1688 (CONC); La Ligua, Quebrada Las Pataguas, $160 \mathrm{~m}$, XII-1958, Escudero s.n. (CONC 75387); Las Palmas de Pedegua, 600 m, X-1945, Moreno s.n. (CONC 116360); Aguas claras cerca de Zapallar, $25 \mathrm{~m}$, 11-II-1953, Levi Heins 175 (CONC); Prov. Quillota, Cerro de la Campana Chica, 29-XII-1937, Barros 195, 2321 (LP); Cerro Roble above Calco ca. $2 \mathrm{~km}$ by trail, $1210 \mathrm{~m}, 2$-XII1951, Hutchinson 67 (GH); Embalse de Los Aromos, 100 m, $32^{\circ} 56^{\prime} \mathrm{S}, 71^{\circ} 20^{\prime} \mathrm{W}, 10-\mathrm{XI}-2001$, Aedo 6806 (CONC); Cuesta

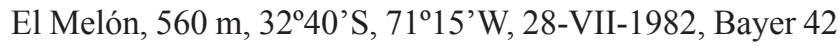
(CONC); ídem, 13-IX-1957, Ricardi \& C. Marticorena 4223 
(CONC); ídem, XI-1969, G. Martínez 49072 (CONC); cerca de La Calera, Cordillera de la Costa, 600 m, 13-XI-1966, Zöllner 1138 (LP); Prov. San Antonio, Rocas de Santo Domingo, 25 m, 24-VII-1955, Gunckel s.n. (CONC 116263); Prov. San Felipe de Aconcagua, Carretera Panamericana, Bajada de El Melón, 13-IX-1957, Cabrera 12528 (LP, M); El Melón, 560 m, II-1953, Collantes s.n. (CONC 75383); Portillo, 2870 m, 6-III-1954, Ricardi 2853 (CONC); Santa Filomena, al interior de Jahuel, $900 \mathrm{~m}, 32^{\circ} 41^{\prime} \mathrm{S}, 70^{\circ} 37^{\prime} \mathrm{W}$, 13-XI-1987, Matthei \& Quezada 463 (CONC); Prov. Valparaíso, Bajada Agua Santa, 9-I-1950, Boelcke 3885 (LP, SI); Las Rocas, 26-XII-1949, Boelcke 3846 (LP, SI); Casablanca, 18-XI-1954, Navas 1389 (CONC); Tunquén,

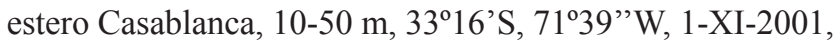
Teillier 5851 (CONC); ca. Placilla de Peñuelas, 360 m, 16XI-1954, Sparre 11016 (CONC); between Maitencillo and Puchuncaví, 26-X-1985, Zöllner 12518 (MO); Marga-

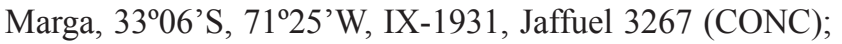
Viña del Mar, 20 m, XI-1953, Gunckel 32025 (CONC); Viña del Mar, El Salto, 110 m, 23-XI-1958, Saa s.n. (CONC 75390); Viña del Mar, Fundo Siete Hermanas, 270 m, 4-XI1936, Behn 24413 (CONC); Recreo Alto, 25 m, 24-XI-1958, Parra 475 (CONC); Granizo, 300 m, 25-X-1959, Weisser Sievers s.n. (CONC 75846); Pucalán, 31-XII-1950, Collantes s.n. (CONC 75384); Cerro Curauma, 3310'5,3"S, 7141'4,86”W, 20-I-2006, Fernández 1494 (CONC); Valle del agua potable, Las Lúcumas, 60 m, 14-XI-1954, Schlegel 433 (CONC); S/Prov., Punta Cristales, Cerro Roble, 1800 m, 29-XI-1965, Zöllner 1069 (CONC). Región Metropolitana de Santiago. Prov. Chacabuco, Panamericana Norte km 22, Straßenrand mit nassem Graben., 3 XII 1966, Zollitsch 22 (M); Prov. Cordillera, inmediaciones Refugio Lo Valdés, Valle de Maipo, 2000 m, 20-XI-1940, Garaventa 1293 (CONC); Cajón del Maipo, Refugio Cruz de Piedra, $34^{\circ} 06^{\prime}$ 'S, 700’W, 2400 m, 20-I-2000, Teillier 4624 (CONC); Prov. Maipo, Cajón de Morales entre las Panimávidas y la laguna, 2200 m, 334'ㄱ, 7004W, 25-I-2002, Teillier \& Márquez 5313 (CONC); ídem, 2150 m, Teillier \& Márquez 5314 (CONC); Laguna de Aculeo, 350 m, 18-V-1971, C. Martínez s.n. (CONC 74364 y 75845); ídem, 11-X-1974, Montero 9437 (CONC); Laguna Negra, ribera oriental, $2720 \mathrm{~m}$, $33^{\circ} 00^{\prime} \mathrm{S}, 6^{\circ} 02^{\prime} \mathrm{W}, 15-\mathrm{I}-1990$, Teillier et al. 2027 (CONC); Valle del Yeso, Estero Salinillas, $2900 \mathrm{~m}, 33^{\circ} 40^{\prime} \mathrm{S}, 69^{\circ} 57^{\prime} \mathrm{W}$, IV-2001, Teillier 6489 (CONC); Prov. Santiago, entre Rungui y La Capilla (Calen), 700-1000 m, 6-XI-1937, Looser 66253 (MO); Comuna de la Reina, Quebrada de

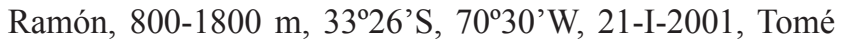
128, 129 (CONC); Valle Macul, 1850 m, XII-1932, Grandjot 995 (CONC); Quebrada de La Plata, 740 m, 3329'S, 7054'W, 15-XII-1960, Schlegel 3329 (CONC); S/Prov., Cuesta de La Dormida, 1200 m, IX-1956, Gunckel 30004 (CONC); ídem, 13-I-1972, Mahu 8657 (LP). Región del Libertador General Bernardo O'Higgins. Prov. Cachapoal, Hacienda de Cauquenes, Cajón del Cypres, 1875, Dessauer s.n. (M 0155925); Reserva Nac. Los Cipreses, $1062 \mathrm{~m}$, $34^{\circ} 15^{\prime} \mathrm{S}, 70^{\circ} 25^{\prime} \mathrm{W}, 2-\mathrm{I}-2006$, A. Marticorena \& Jiménez s.n. (CONC 173679); Pangal, 1000 m, 25-XI-1978, Zöllner 10151 (CONC); Termas de Cauquenes, camino a Coya, 800 m, 4-XI-1952, Pfister s.n. (CONC 13160); Prov. Colchagua, Laguna Cahiul, 50 m, 12-II-1954, Arriaga s.n. (CONC 75391). Región del Maule. Prov. Curicó, La Montaña, Teno, 27-I-1945, Barros 3874 (CONC); Valle Vergara, 920 km del límite entre Chile- Argentina, II-1967, Calderón s.n. (CONC 34579). Región del Biobío. Prov Nuble, Puente El Roble, 64 m, 36²5'S, 72²5’W, 14-XI-1972, Igaymán \& M. Muñoz s.n. (CONC 89695). Región de Los Lagos. Prov. Llanquihue, Petrohué, 210 m, 18-I-1979, Zöllner 10198 (CONC). Región de Aysén del General Carlos Ibáñez del Campo. Prov.

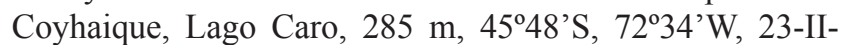
2007, García 4083 (CONC). Región de Magallanes y de la Antártica Chilena. Prov. Última Esperanza, Puerto Prat, Seno Última Esperanza, 26-I-1904, Hicken 122 (SI).

6. Pseudognaphalium lacteum (Meyen \& Walp.) Anderb., Opera Bot. 104: 147. 1991. Gnaphalium lacteum Meyen \& Walp., Nov. Actorum Acad. Caes. Leop.-Carol. Nat. Cur. 19 (Suppl. 1): 276. 1843. TYPE: Perú. Meyen s.n. (holotype, $\mathrm{B}$ (probably destroyed), = photo F15105!; lectotype, here designated, GH 00008360, = photo! fragment ex B). Fig. 10.

Gnaphalium argyrolepis Phil., Anales Mus. Nac., Santiago de Chile 8: 46. 1891. TYPE: Chile. Iquique. Usmagama, 15III-1885, C. Rahmer s.n. (lectotype, here designated, SGO 64432!).

Dwarf perennial herbs, $2-8 \mathrm{~cm}$ tall, multistemmed, stems prostrate, branched, whitish woolly. Leaves approximate, ca. $15 \times 3 \mathrm{~mm}$, obovate, margin flat, apex rounded, base atenuate; concolorous, woolly on both surfaces, trichomes of 2 types, long eglandular eseptate, 4-celular, and few biseriate glandular trichomes hidden under the wool. Capitula few, in clusters arranged in corymbs or panicles. Involucre broadly campanulate, ca. $5 \times 5 \mathrm{~mm}$. Phyllaries 4-seriate, lamina usually opaque milky-white, rounded; outer phyllaries ca. $4 \times 2-2.7 \mathrm{~mm}$, broadly elliptic; inner phyllaries $4-4.6 \times 0.7-1 \mathrm{~mm}$, oblong-lanceolate. Pistillate florets 42-59, corolla yellow, filiform, ca. $3.8 \mathrm{~mm}$ long. Bisexual florets 8-15, corolla yellow, tubular, ca. $3.7 \mathrm{~mm}$ long. Achenes $0.6-1 \mathrm{~mm}$ long, glabrous, epidermis smooth. Pappus bristles ca. 3.2. $\mathrm{mm}$ long.

Phenology. Flowering from September to May.

Distribution and habitat. Perú (Dillon \& Sagástegui 1991), Bolivia (Hind 2011), NW Argentina (Catamarca, Jujuy, Salta) and northern Chile (Arica y Parinacota, Tarapacá, Coquimbo, Biobío), between 3000-4800 m. 

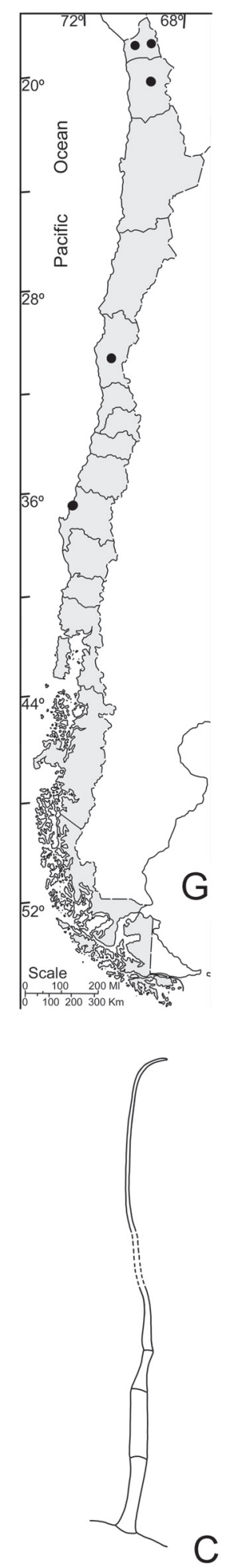

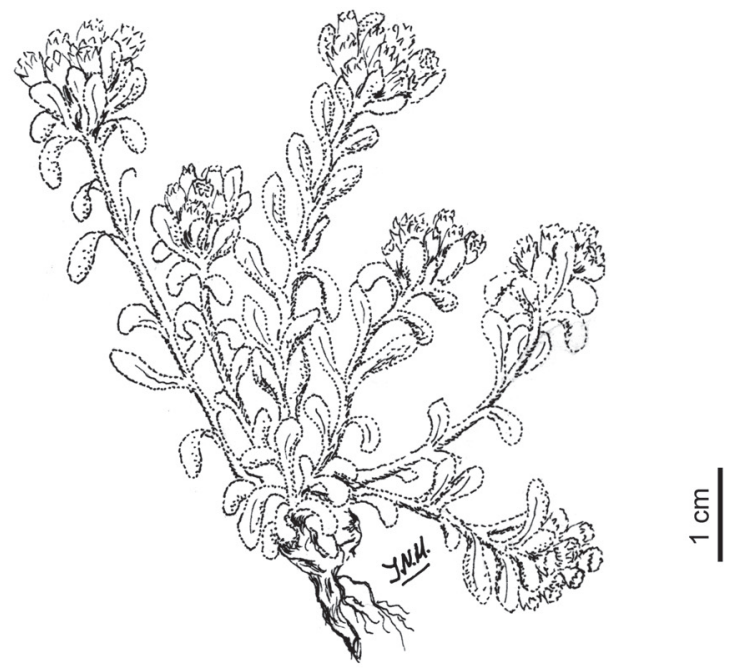

A
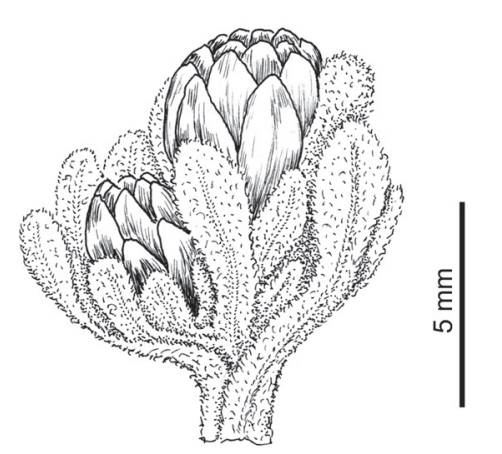

B

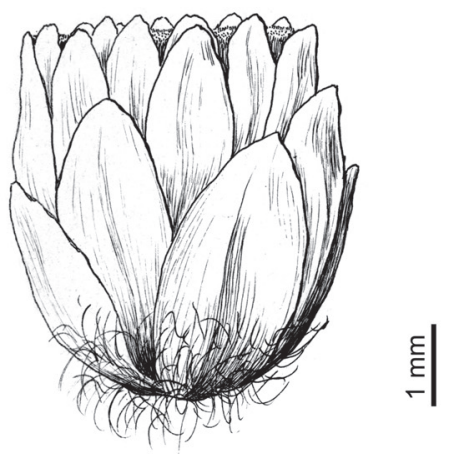

$E$
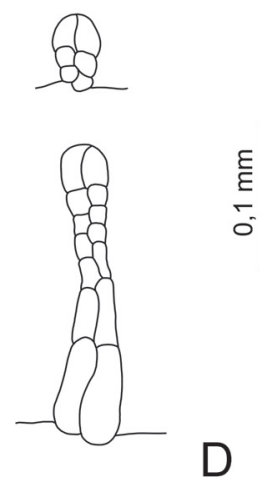
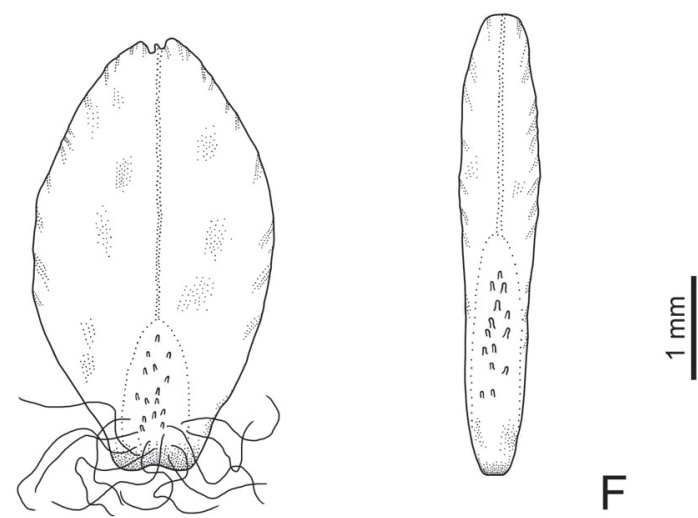

Figure 10. Pseudognaphalium lacteum. A, Habit; B, Cluster of capitula and leaves; C, D, Foliar trichomes; E, Capitulum; F, Phyllaries; G, Distribution [A, B, E, from Cabrera 1978; C, D, Werdermann 1092, LP; F, Antoniz s.n., CONC].

Figura 10. Pseudognaphalium lacteum. A, Planta; B, Detalle de la inflorescencia; C, D, Tricomas foliares; E, Capítulo; F, Filarios; G, Distribución [A, B, E, tomado de Cabrera 1978; C, D, Werdermann 1092, LP; F, Antoniz s.n., CONC]. 
Relationships. Pseudognaphalium lacteum appears to occupy an isolated position among Chilean species taking in consideration its dwarf habit with prostrate branched stems and usually opaque milky-white phyllaries.

\section{ObSERVATIONS}

1. Because the collection mentioned in the protologue of Gnaphalium lacteum, 'Peruvia: in planitie circa Tacoram, alt 14-17000 ped.' and deposited at B was probably destroyed, the isotype at GH (fragment), which is sufficiently diagnostic, is designated as lectotype.

2. Two specimens from Tarapacá are mentioned in the protologue of Gnaphalium argyrolepis: 'Habitat in provincia Tarapacá ad Usmagama, Vilon, etc.'. We found at SGO three sheets, two from Vilon: [Vilon, pr. Atacama, 17-II-1885, Philippi s.n.] SGO 44934, 71282, and the other from Usmagama, [Usmagama, 15-III-1885, C. Rahmer s.n.] SGO 64432. We propose the material SGO 64432, which presents the most complete plant, as lectotype of Gnaphalium argyrolepis.

Additional material examined. CHILE. Región de Arica y Parinacota. Prov. Arica, Cordillera de Arica, Cuesta de Chapiquiña, cerca del portezuelo, 4000 m, 29-III-1962, Ricardi et al. 325 (CONC); camino de Arica al Portezuelo de Chapiquiña, km 92, 3250 m, 8-II-1964, C. Marticorena et al. 14 (CONC); FF.CC. de Arica-La Paz, Puquios, km 106-108, 3600-3700 m, 27-IX-1955, Ricardi \& Silva 3544 (BAB, CONC). Prov. Parinacota, cerca de Parinacota, $4400 \mathrm{~m}, 18^{\circ} 13^{\prime} \mathrm{S}, 6^{\circ} 14^{\prime} \mathrm{W}, 8-\mathrm{III}-1984$, Arroyo 84-691 (CONC); Putre, 3500 m, 12-II-1964, C. Marticorena et al. 142 (CONC); camino Zapahuira-Putre, Quebrada Chilcana, $3400 \mathrm{~m}, 18^{\circ} 14^{\prime} \mathrm{S}, 6^{\circ} 33^{\prime} \mathrm{W}, 15-\mathrm{III}-1985$, Landero et al. 48 (CONC); Lago Chungará, arriba guardería CONAF, 4600 m, 18 ${ }^{\circ} 14^{\prime} \mathrm{S}, 6^{\circ} 10^{\prime} \mathrm{W}, 18-\mathrm{V}-1989$, Niemeyer et al. 89106 (CONC); Guaneguane, bofedal. $4450 \mathrm{~m}, 18^{\circ} 10^{\prime} \mathrm{S}, 69^{\circ} 15^{\prime} \mathrm{W}$, Niemeyer et al. 89101 (CONC); Lagunas de Cotacotani, 4500 m, 13-II-1964, C. Marticorena et al. 244 (CONC); camino de Chucuyo a las Lagunas de Cotacotani, km 5, 4400 m, 13-II-1964, C. Marticorena et al. 227 (CONC). Región de Tarapacá. Prov. El Tamarugal, Cerro Japu, 4200 m, III1926, Werdermann 1092 (CONC, NY, LP, SI); Tranque de Caritaya, 3600 m, 30-IX-1958, Ricardi \& C. Marticorena 4807 (CONC); camino de Huara a Cancosa, km 91, 3850 m, 17-II-1964, C. Marticorena et al. 316 (CONC). Vilon, pr. Atacama, 17-II-1885, Philippi s.n. (SGO 44934, 71282). Región de Coquimbo. Prov. Limarí, Carretera Panamericana, $19 \mathrm{~km}$ al S de Socos, 300 m, 9-II-1963, Ricardi et al. 788 (CONC); Cordillera de Combarbalá, Potrero Grande, 2800 m, 31 10' $\mathrm{S}$, 7050’W, 11-I-1973, Jiles 6083 (CONC).
Región del Biobío. Prov. Concepción, San Pedro, X-1952, Antoniz s.n. (CONC 13265).

7. Pseudognaphalium landbeckii (Phil.) Anderb., Opera Bot. 104: 147. 1991. Gnaphalium landbeckii Phil., Linnaea 33: 165. 1864. TYPE: CHILE. Colchagua, Llico, XII-1861, Lanbeck s.n. (lectotype, here designated, SGO 64383!; isolectotypes, LP 116929!, SGO 44952!). Fig. 11.

Perennial herbs, $15-30 \mathrm{~cm}$ tall, stem solitary (2 or 3$)$, erect, unbranched, whitish woolly. Stems leaves 35-40 $\times$ 5-7 mm, broadly oblong, margin flat, apex obtuse, base decurrent 6-8 $\mathrm{mm}$ long; basal leaves 50-70 × 9-10 mm, linear-obovate; concolorous, woolly, trichomes of 2 types, long eglandular eseptate trichomes, 3-4-celular, and few biseriate glandular trichomes hidden under the wool. Capitula numerous in clusters arranged in dense corymbs. Involucre campanulate, 4-5 × $5 \mathrm{~mm}$. Phyllaries ca. 42, 4-seriate, lamina brown, hyaline and shiny; outer phyllaries 3-4 × 1.5-2.7 mm, obovate, apex subobtuse, lanuginose; inner phyllaries 3.1$4.6 \times 0.7-1.3 \mathrm{~mm}$, linear, apex acute, glabrous. Pistillate ca. 180, corolla yellow, filiform, ca. $2.5 \mathrm{~mm}$ long. Bisexual florets ca. 14, corolla yellow, tubular, ca. $2.7 \mathrm{~mm}$ long. Achenes, 0.6-0.7 mm long, glabrous, epidermis smooth or papillose. Pappus bristles 3-3.2 mm long.

Phenology. Flowering from October to December (February).

Distribution and habitat. Endemic to Chile (Valparaíso, Metropolitana, Maule), between 50-700 m.

Relationships. Pseudognaphalium landbeckii seems to be distinguished by its usually solitary stems, numerous capitula in clusters arranged in dense corymbs, and stem leaves with broadly oblong leaf blades.

\section{Observation}

There is a photo-Field Museum Nr.15106, with a specimen collected in Chillán. Since the protologue of Gnaphalium landbeckii mentions 'Llico' this specimen is excluded from the type collection of $G$. landbeckii.

Additional material EXAmined. CHILE. Región de Valparaíso. Prov. Valparaíso, Concón, 7-X-1953, Cabrera 11462 (LP). Región Metropolitana de Santiago. Prov. Santiago, Clarillo, X-1958, Gunckel 46477 (CONC). Región del Maule. Prov. Curicó, Llico, II-1950, Ojeda 20.949 (CONC); Prov. Talca, Constitución, Playa grande, 1-XI-1958, Barnier 488 (CONC). 

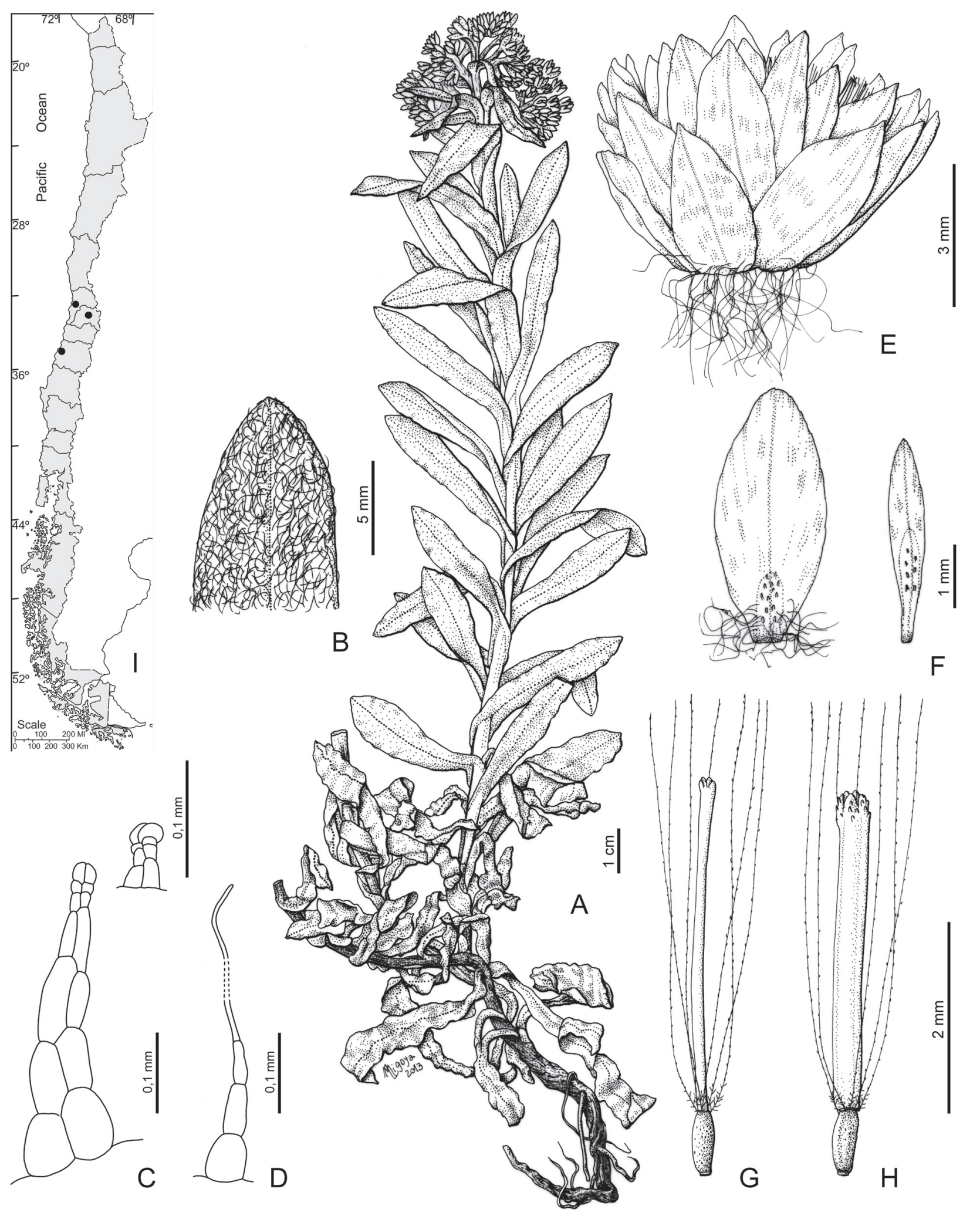

Figure 11. Pseudognaphalium landbeckii. A, Habit; B, Leaf pubescence; C, D, Foliar trichomes; E, Capitulum; F, Phyllaries; G, Pistillate floret; H, Bisexual floret. I, Distribution [A, B, E, G, H, Landbeck in XII-1861, LP; C, D, F, Cabrera 11462, LP].

Figura 11. Pseudognaphalium landbeckii. A, Planta; B, Pubescencia de la hoja; C, D, Tricomas foliares; E, Capítulo; F, Filarios; G, Flor pistilada; H, Flor perfecta; I, Distribución [A, B, E, G, H, Landbeck en XII-1861, LP; C, D, F, Cabrera 11462, LP]. 
8. Pseudognaphalium munoziae N. Bayón, C. Monti \& S.E. Freire, Phytotaxa 105(1): 1-10. 2013. TYPE: Chile: Región Arica-Parinacota: Subida a Portezuelo de Chapiquiña, $18^{\circ} 20^{\prime} 1.7^{\prime \prime} \mathrm{S}, 69^{\circ} 31^{\prime} 1^{\prime \prime} \mathrm{W}, 4.147 \mathrm{~m}, 5-\mathrm{V}-2011$, A. Moreira, M. Muñoz \& V. Morales 1676 (holotype SGO!). Fig.12.

Perennial herbs, 4-6(15) cm tall, multistemmed, stems erect or ascending, unbranched, arachnoid-glandulose. Stem leaves remote, 10-25(30-40) × 1(2) $\mathrm{mm}$, linear, margin flat, apex acute to subobtuse, base attenuate; basal leaves rosulate, $20-50(70-75) \times 1-2 \mathrm{~mm}$, linear, apex acute to subobtuse, base long-attenuate; concolorous, greenish, arachnoid-glandular on both surfaces, trichomes of 2 types, long eglandular eseptate and long (short) glandular biseriate trichomes. Capitula numerous, in clusters arranged in narrow panicles, clusters subtended by linear leaves. Involucre 5-6 $\times$ 4-7 mm, campanulate. Phyllaries 3-4-seriate, lamina opaque milky-white; outer phyllaries 4-5 × 1-2 mm, narrowly ovate, apex obtuse; inner phyllaries 5-6 $50.7-1$ $\mathrm{mm}$, ovate-oblong, apex obtuse. Pistillate florets 41-45; corollas whitish, filiform, ca. $3 \mathrm{~mm}$ long. Bisexual florets ca. 6, corollas yellowish at apex, tubular, 3.5-3.8 mm long. Achenes ca. $0.3 \mathrm{~mm}$ long, glabrous, epidermis papillose above. Pappus bristles 3.5-4 mm long.

Phenology. Flowering in May, June and October.

Distribution AND habitat. Endemic to northern Chile (Arica y Parinacota, Tarapacá), between 3500-4200 m.

ReLATIONSHiPs. Pseudognaphalium munoziae is most similar to P. psilophyllum. Both have campanulate involucres and glandular linear leaves. Pseudognaphalium munoziae is recognized by its conspicuously rosulate basal leaves and shorter upper leaves, all apically acute to subobtuse, and capitula arranged in narrow panicles with milky-white phyllaries. Pseudognaphalium psilophyllum is distinguished by its similar basal and upper leaves, which are attenuate and acute at the apex, and capitula arranged in corymbs or corymbose-paniculate with usually brown phyllaries

Additional material examined. CHILE. Región de Arica y Parinacota. Prov. Parinacota, inicio de bajada a Quebrada Allane, $18^{\circ} 0^{\prime} 7^{\prime \prime} \mathrm{S}, 69^{\circ} 37^{\prime} 14^{\prime \prime} \mathrm{W}, 3550 \mathrm{~m}, 10$-VI2012, Moreira 1953 (SGO). Región de Tarapacá. Prov. El Tamarugal, trayecto entre Enquelga y Berenguela, 9-X1997, Villagrán et al. 9229 (CONC).

9. Pseudognaphalium psilophyllum (Meyen \& Walp.) Anderb., Opera Bot. 104: 147. 1991. Gnaphalium psilophyllum Meyen \& Walp., Nov. Actorum Acad. Caes.
Leop. Carol. Nat. Cur. 19 (Suppl. 1): 275. 1843. TYPE: Chile. Región de Valparaíso. Prov. Marga Marga, Limache, 9-XI-1927, 900 m s.m., Garaventa 1151 (neotype, designated here, CONC!). Fig. 13.

Gnaphalium glandulosum Klatt, Linnaea 42: 129. 1878. Pseudognaphalium glandulosum (Klatt) Anderb., Opera Bot. 104: 147. 1991, syn. nov. TYPE: Chile. Parinacota, Tacora, F.J.F Meyen s.n. (holotype, B (probably destroyed), = photo F15099!; lectotype, here designated, GH 00008359, $=$ photo! fragment ex Herb. Klatt).

Perennial herbs, (5)10-85 cm tall, multistemmed, rarely solitary, erect or ascending, usually branched at the upper part, arachnoid-glandular. Stem leaves (7)10-65 × 1-6 $\mathrm{mm}$, stem leaves linear, margin flat and usually revolute, apex long-attenuate, acute, base not broad, decurrent 4-10 mm long; basal leaves $85-90 \times 4-8 \mathrm{~mm}$, linear-obovate; concolorous, glandular on both surfaces, sometimes arachnoid on the abaxial surface, trichomes of 2 types, long (short) biseriate glandular trichomes and few long eglandular eseptate, 3-6-celular trichomes. Capitula numerous in clusters arranged in corymbs or panicles. Involucre broadly campanulate, 3-5 × 3-5 mm. Phyllaries 4-seriate, lamina straw-yellow to brown, hyaline and shiny, apex semiobtuse; outer phyllaries $4.5-5 \times 1.8-2.6 \mathrm{~mm}$; inner phyllaries $3.7-$ $4.5 \times 0.6-1.7 \mathrm{~mm}$, oblong-obovate, apex acute. Pistillate florets (46)82-143, corolla yellow, filiform, 2.5-3 mm long. Bisexual florets 5-14, corolla yellow, tubular, 3-3.2 mm long. Achenes $0.6-1 \mathrm{~mm}$ long, glabrous, epidermis smooth to papillose. Pappus bristles $2.5-3.4 \mathrm{~mm}$ long.

Phenology. Flowering from September to May.

Distribution and habitat. Bolivia (Hind 2011), Argentina (Jujuy, Salta, Catamarca, La Rioja, Mendoza, Neuquén, Río Negro) and Chile (Arica y Parinacota, Tarapacá, Antofagasta, Coquimbo, Valparaíso, Libertador General Bernardo O'Higgins, Maule, Los Lagos), growing between (5-50) 900-4500 m.

Relationships. Pseudognaphalium psilophyllum resembles to P. munoziae. Both species are glandular or glandulararachnoid plants and have linear leaves. Pseudognaphalium psilophyllum can be distinguished by its similar basal and upper leaves (vs. conspicuously rosulate basal leaves and shorter upper leaves in P. munoziae) and capitula in clusters arranged in corymbs or corymbose-paniculate with usually brown phyllaries (vs. capitula arranged in narrow panicles with milky-white phyllaries). 

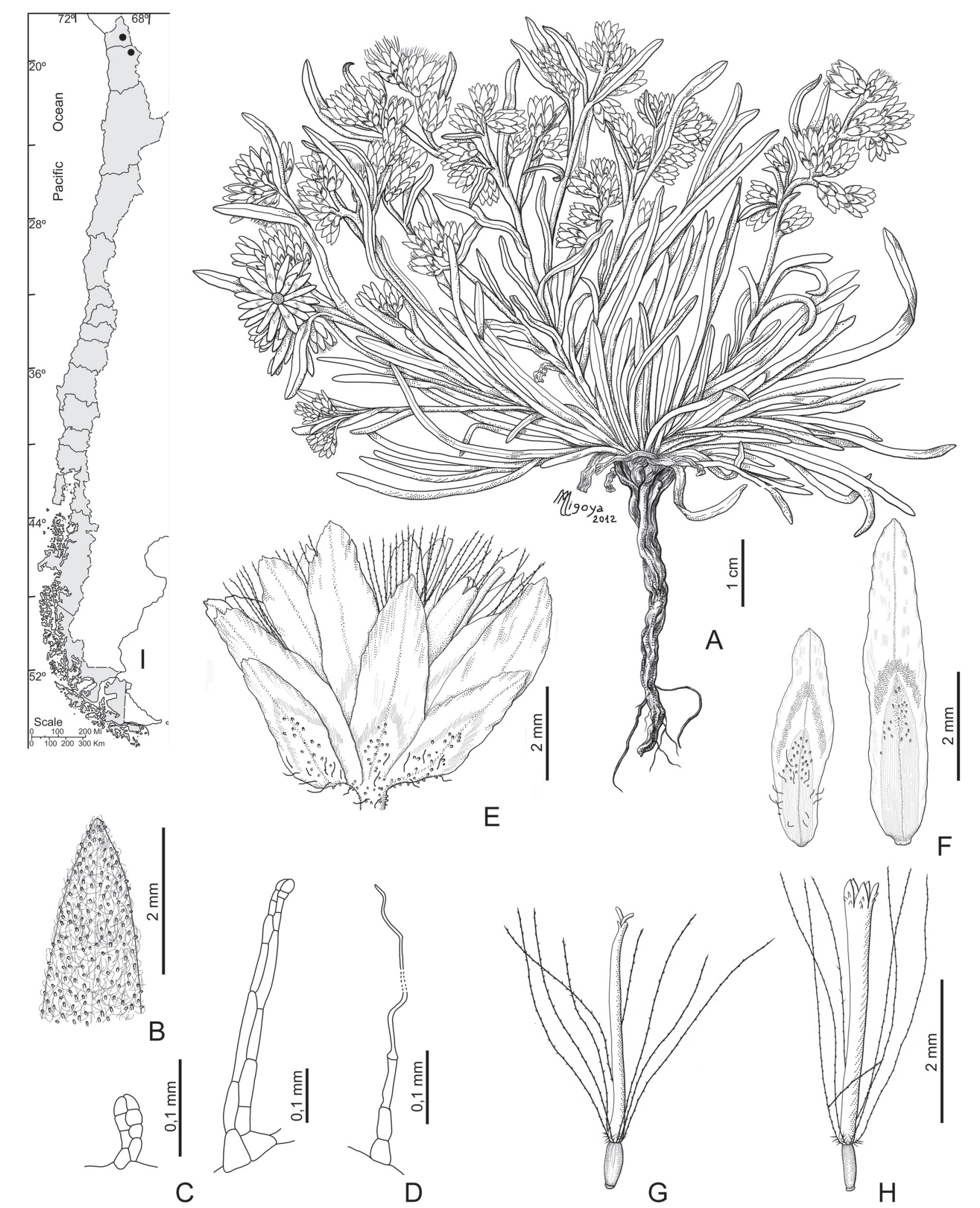

Figure 12. Pseudognaphalium munoziae. A, Habit; B, Leaf pubescence; C, D, Foliar trichomes; E, Capitulum; F, Phyllaries; G, Pistillate floret; H, Bisexual floret. I, Distribution [A-H, from Freire et al. 2013].

Figura 12. Pseudognaphalium munoziae. A, Planta; B, Pubescencia de la hoja; C, D, Tricomas foliares; E, Capítulo; F, Filarios; G, Flor pistilada; H, Flor perfecta; I, Distribución [A-H, tomado de Freire et al. 2013]. 

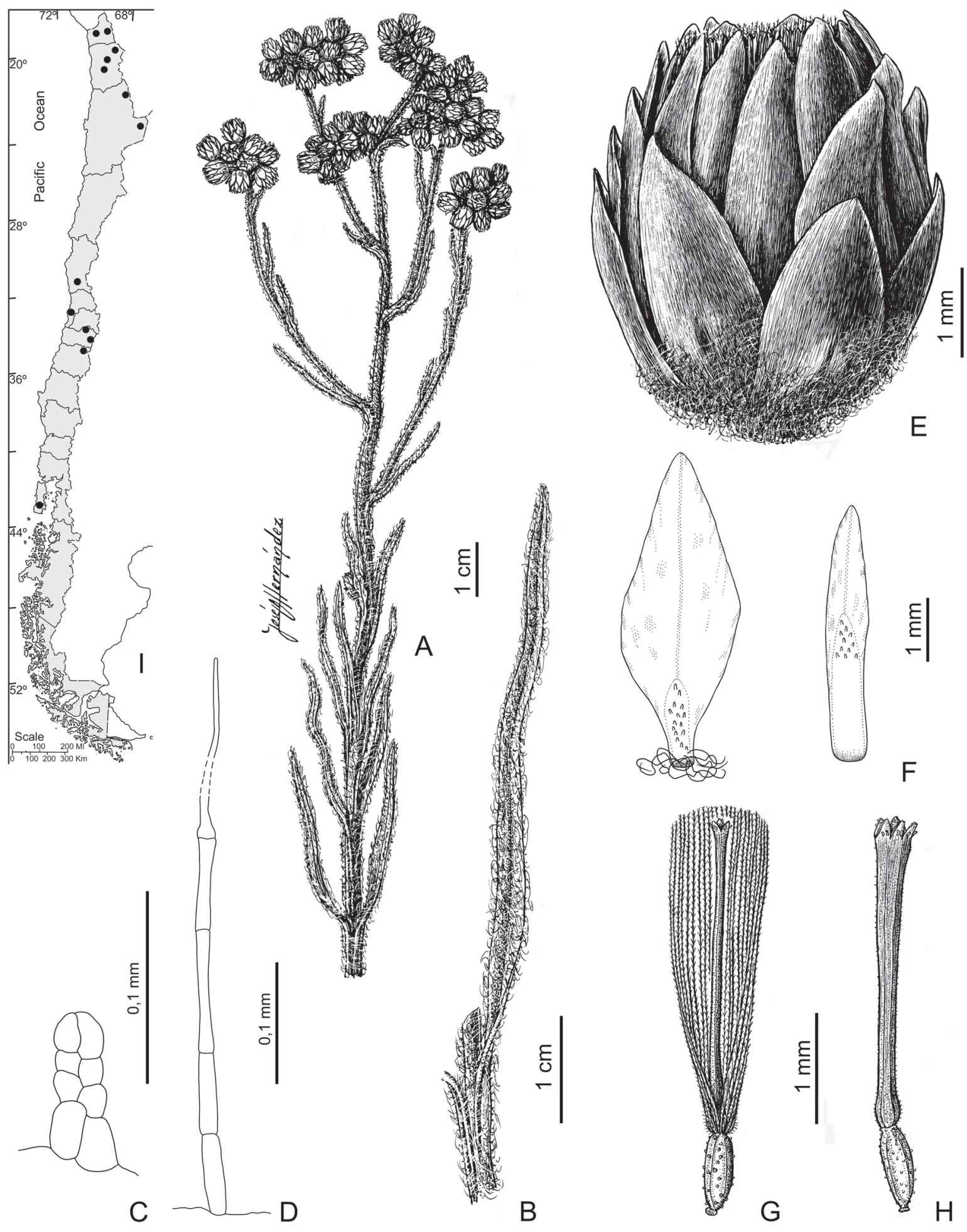

Figure 13. Pseudognaphalium psilophyllum. A, Apical flowering stem; B, Leaf ; C, D, Foliar trichomes; E, Capitulum; F, Phyllaries; G, Pistillate floret; H, Bisexual floret without pappus; I, Distribution [A, B, E, G, H from Cabrera 1971; C, D, Spegazzini s.n., BAB; F, Niemeyer et al. 8970, CONC].

Figura 13. Pseudognaphalium psilophyllum. A, Parte superior de la planta; B, Hoja; C, D, Tricomas foliares; E, Capítulo; F, Filarios; G, Flor pistilada; H, Flor perfecta sin el papus; I, Distribución [A, B, E, G, H tomado de Cabrera 1971; C, D, Spegazzini s.n., BAB; F, Niemeyer et al. 8970, CONC]. 


\section{OBSERVATIONS}

1. The types designated in the protologue of Gnaphalium psilophyllum Meyen \& Walp., '(Besser!) cum praecedente (Meyen!)', have not been located at B (probably destroyed), GOET, M, or W, no illustration was published with the name, and no other original material has been found. The specimen 'Garaventa 1151' kept at CONC, which is in accordance with the protologue and matches the locality ('cum praecedente', i.e. Valparaiso in Gnaphalium dysodes Spreng.) indicated in the protologue, is here selected as the neotype of $G$. psilophyllum.

2. Klatt (1878) described Gnaphalium glandulosum on the basis of its 'caule fruticoso ascendente erecto ramoso follisque glandulosis, foliis lanceolato-linearibus acuminatis semiamplexicaulibus, ramis paniculato-corymbosis, capitulis conglomeratis, involucri campanulati basi lanati squamis obtusis pallide-fuscescentibus'. Gnaphalium glandulosum is proposed here as a new synonym of $G$. psilophyllum because there is no diagnostic character that differentiates the former from the latter. Both have erect or ascending stems that are arachnoid-glandular, caulinar leaves which are linear, attenuate at the apex, decurrent at the base, concolorous, glandular on both surfaces, and capitula in clusters arranged in corymbs or panicles.

Additional material examined. CHILE. Región de Arica y Parinacota. Prov. Arica, camino de Arica al Portezuelo de Chapiquiña, km 93, 3350 m, 8-II-1964, C. Marticorena et al. 21 (CONC); camino de Azapa a Chapiquiña, km 76, 3250 m, 24-IX-1958, Ricardi \& C. Marticorena 4730 (CONC); FF.CC. de Arica-La Paz, Puquios, km 106-108, 3600-3700 m, 27-IX-1955, Ricardi \& Silva 3535 (CONC, LP). Prov. Parinacota, Socoroma, 3200 m, 2-II-2000, Belmonte 20060 (CONC); Putre, a 4 km, 18 $12^{\prime} \mathrm{S}-69^{\circ} 34^{\prime} \mathrm{W}, 3500 \mathrm{~m}, 16-\mathrm{V}-$ 1989, Niemeyer et al. 8970 (CONC); Putre, 3500 m, 12II-1964, C. Marticorena et al. 141 (CONC); Putre, about 4-6 km below turnoff to Putre on road to Arica, ca. 3500 m, 29-XII-1995, Landrum \& Landrum 8887 (MO); camino a Chapiquiña, quebrada frente a la central hidroeléctrica, 3400 m, 8-V-1972, Ricardi et al. 286 (CONC); cordillera de Arica, camino de Parinacota a Lago Chungará, 4200 m, 29-III-1961, Ricardi et al. 311 (CONC); entre Zapahuira y Putre, 3270-3600 m, 15-IV-1980, Villagrán et al. 2441 (CONC); camino de Arica a Zapahuira, $2900 \mathrm{~m}, 18^{\circ} 28^{\prime} \mathrm{S}$, 6945'W, 13-IV-1980, Villagrán et al. 2353 (CONC); entre Zapahuira y Putre, 3270-3600 m, 18¹8'S, 69³4'W, 15-IV1980, Villagrán et al. 2478 (CONC); Socoroma, 3200 m, 12-VII-1982, Zöllner 11254 (MO). Región de Tarapacá. Prov. El Tamarugal, camino de Huara a Cancosa, cerca de Coscaya, 3200 m, 2-IV-1961, Ricardi et al. 398 (CONC); trayecto entre Enquelga y Berenguela, $4200 \mathrm{~m}, 19^{\circ} 15^{\prime} \mathrm{S}$, 6908'W, 9-IX-1997, Villagrán et al. 9237 (CONC); Prov. Iquique, Quebrada de Chusmiza, 1941'S-69²11'W,
4-IX-1947, Villagrán et al. 9098 (CONC); Cordillera Cerro Japu, 4000 m, III-1926, Wedermann 1100 (CONC, LP, SI); Interior de las Termas de Mamiña, 3200 m, 4-II1965, Zőllner 976 (LP). Región de Antofagasta. Prov. El Loa, Quebrada Del Inca, Cerro Aucanquilcha, $4500 \mathrm{~m}$, 2114'S, 68²8'W, 2-IV-1985, Arroyo 85-586 (CONC); camino de San Pedro de Atacama a Paso Jama, límite entre pastizal y tolar, 4200 m, 4-IV-1997, Arroyo et al. 97069 (CONC), transecto Cerro Carasilla a Sala Ascotán, 4350 m, 28-III-1985, 21 $41^{\circ} \mathrm{S}, 68^{\circ} 17^{\prime} \mathrm{W}$, Arroyo et al. 85-393 (CONC). Región de Coquimbo. Prov. Choapa, in Potrero Largo near Cuncumén, 2700 m, 6-II-1984, Zöllner 11991 (MO); Alrededores de Toconce, terrazas de cultivo, 3300 $\mathrm{m}, 22^{\circ} 15^{\prime} \mathrm{S}, 68^{\circ} 10^{\prime} \mathrm{W}, 26-\mathrm{I}-1980$, Villagrán \& Armesto 2271 (CONC); Illapel, Palo Colorado, pleno campo, $10 \mathrm{~m}$, 3204'S, 71³1'W, 10-XI-1961, Jiles 3887 (CONC); Prov. Elqui, Carretera Panamericana entre Pichidangui y Los Vilos, 12-X-1963, C. Marticorena \& Matthei 49 (CONC); ibid., C. Marticorena \& Matthei 67 (CONC). Región de Valparaíso. Prov. Valparaíso, Algarrobo, 20 m, XI-1965, Semler s.n., Herb. Gunckel 48696 (CONC 116219); Tunquén, estero Casablanca, 10-50 m, 3316'S, 71³9'W, 1-XI-2001, Teillier 5915 (CONC). Región del Libertador General Bernardo O'Higgins. Prov. Cachapoal, valle superior Río Cachapoal, 1500 m, X-1978, Prov. Colchagua, Termas de El Flaco, 23-I-1948, Barros 7332 (LP). Región del Maule. Prov. Curicó, Cord. del Planchón, 17-II-1939, Barros 2303 (SI). Región de Los Lagos. Prov. Chiloé, isla Surgidero, $43^{\circ} 25^{\prime} \mathrm{S}-7^{\circ} 14^{\prime} \mathrm{W}, 5-30$ m, 9-II-1986, Villagrán \& Leiva 7448 (CONC).

10. Pseudognaphalium remyanum (Phil.) Anderb., Opera Bot. 104: 147. 1991. Gnaphalium remyanum Phil., Anales Univ. Chile 90: 8. 1895. TYPE: Chile. Santiago, XII-1877, Philippi s.n. (lectotype, here designated SGO 44975!; isolectotypes, B (probably destroyed), = photo F15113!, SGO 64436!). Fig. 14.

Perennial herbs, ca. $50 \mathrm{~cm}$ tall, stem branched above base, whitish-arachnoid. Leaves up to $50 \times 8 \mathrm{~mm}$, linear, margin flat, apex acuminate, base decurrent 3-4 $\mathrm{mm}$ long, and sometimes broad; discolorous, adaxial surface green, abaxial surface whitish, arachnoid on both surfaces, trichomes of 2 types, long eglandular eseptate, 3-celular, and few short biseriate glandular trichomes. Capitula numerous in clusters arranged in corymbs or lax panicles. Involucre broadly campanulate, 2.2-2.5 × 2-4 mm. Phyllaries 3-4-seriate, lamina yellowish to whitish, hyaline and shiny; outer phyllaries ca. $2 \times 1.6 \mathrm{~mm}$, broadly ovate, apex rounded; inner phyllaries ca. $2 \times 0.8 \mathrm{~mm}$, oblongobovate, apex obtuse. Pistillate florets ca. 163, corolla yellow, filiform, $1.2 \mathrm{~mm}$ long. Bisexual florets ca. 13, corolla yellow, tubular, $1.5 \mathrm{~mm}$ long. Achenes ca. $0.3 \mathrm{~mm}$ 

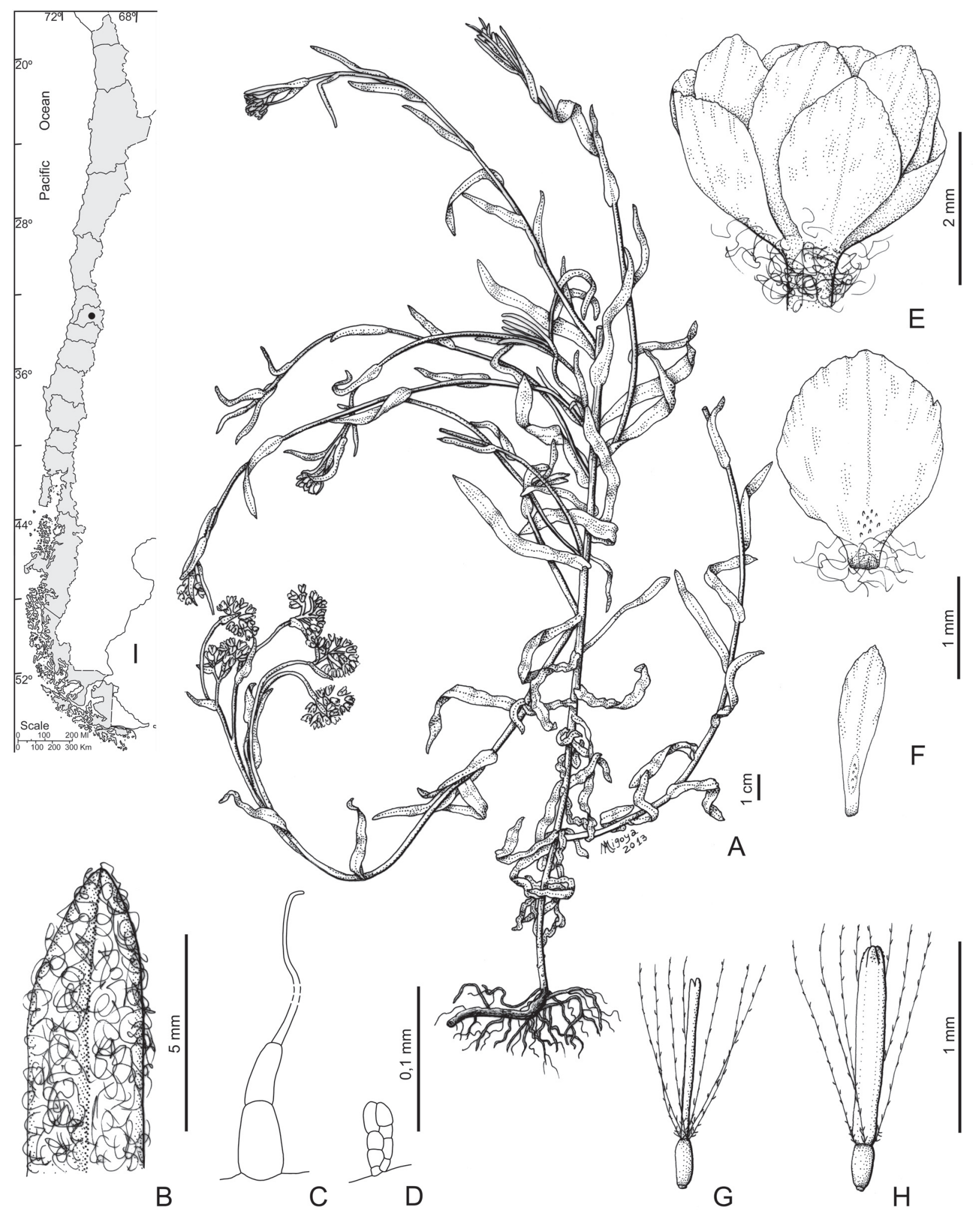

Figure 14. Pseudognaphalium remyanum. A, Habit; B, Leaf pubescence; C, D, Foliar trichomes; E, Capitulum; F, Phyllaries; G, Pistillate floret; H, Bisexual floret. I, Distribution [A, E-H, Philippi in 1877, SGO; B-D, Buchtien 174, NY].

Figura 14. Pseudognaphalium remyanum. A, Planta; B, Pubescencia de la hoja; C, D, Tricomas foliares; E, Capítulo; F, Filarios; G, Flor pistilada; H, Flor perfecta; I, Distribución [A, E-H, Philippi en 1877, SGO; B-D, Buchtien 174, NY]. 
long, glabrous, epidermis papillose. Pappus bristles 1.3-1.7 mm long.

Phenology. Flowering in December.

Distribution and habitat. Bolivia and Central Chile (Región Metropolitana).

Relationships. Pseudognaphalium remyanum seems to be distinguished by its whitish arachnoid plants, linear leaves, and capitula in clusters arranged in lax corymbs or panicles with broadly ovate outer phyllaries.

\section{OBSERVATION}

Although no Chilean material was available of Pseudognaphalium remyanum, it closely resembles the genus Pseudognaphalium in habit, leaves, floral characters and arrange of its capitula, so the species is referred here, until further study.

Additional material examined. BOLIVIA. Sur-Yungas, Sirupaya vec. de Yanacachi, 14-XII-1906, O. Buchtien 174 (NY).

11. Pseudognaphalium tarapacanum (Phil.) Anderb., Opera Bot. 104: 148. 1991. Gnaphalium tarapacanum Phil., Anales Mus. Nac., Santiago de Chile 8: 46. 1891. TYPE: Chile. Tarapacá, Paroma 25-II-1885, F. Philippi s.n., Calcahuay, I-1886, Rahmer s.n. (lectotype, here designated, SGO 64433! - entire plant). Fig. 15.

Perennial herbs, $10-20 \mathrm{~cm}$ tall, multistemmed, stems erect or ascending, unbranched or branched at the upper part, arachnoid-glandulose. Stem leaves ca. $40 \times 4-6$ $\mathrm{mm}$, lanceolate, margin flat, apex attenuate, base broad and decurrent 5-7 mm long; basal leaves oblanceolatespathulate, apex acute or subotuse; concolorous, arachnoidglandulose on both surfaces, trichomes of 2 types, long eseptate eglandular trichomes, 5-8 celular and few short biseriate glandular trichomes. Capitula numerous arranged in dense terminal clusters. Involucre broadly campanulate, 3-5 × 3-4 mm. Phyllaries 3-4-seriate, lamina light brown, hyaline and shiny, apex rounded; outer phyllaries $3-5 \times$ (0.5)1.5-2 mm, elliptic; inner phyllaries 3-4 × 0.5-1 mm, oblong. Pistillate florets $66-151$, corolla yellowish, filiform, 2-3 mm. Bisexual florets 5-11, corolla yellowish, tubular, 2-3 mm. Achenes 0.5-0.9 mm long., pilose, with short duplex hairs or glabrous with epidermis papillose. Pappus bristles, 2-3 mm long.

Phenology. Flowering from (November) January to May.

Distribution AND HABITAT. Northwestern Argentina (Catamarca, Córdoba, Jujuy, La Rioja, Salta, San Luis,
Tucumán) and northern-central Chile (Arica y Parinacota, Tarapacá, Antofagasta, Coquimbo, Metropolitana, Maule, Biobío), growing between (20/300-800) 2600-3500 m.

Relationships. Pseudognaphalium tarapacanum resembles P. psilophyllum. Both species are glandular or glandulararachnoid plants. Pseudognaphalium tarapacanum is easily distinguished by its stem leaves broad at base and occasionally undulate leaf blade margins base (vs. stem leaves not broad at base and flat leaf blade margins in $P$. psilophyllum).

\section{OBSERVATION}

The protologue of Gnaphalium tarapacanum mentions two specimens from Tarapacá Province, i.e. 'Habitat in provincia Tarapacá ad Paroma et Calcahuay'. We located at LP the collection Philippi s.n., Paroma, 25 II 1885 LP 001945, at SGO one sheet-SGO 64433 with two specimens (an entire plant, on the right hand side and a fragment, on the left hand side) mounted on it, and two labels on the right-hand side, i.e. 'Calcahuay, Januario 1886, Rahmer' and 'Paroma, 25 II 1885, F. Ph', and a photo F15120 'Tarapacá, Phil.' . We propose the specimen kept at SGO as the lectotype where the original herbarium of Philippi is kept, and which represent the most complete plant. Since the collector and collection number can not be assigned with certainty to each mounted specimen, we selected as lectotype of Gnaphalium tarapacanum the plant on the right hand of the sheet.

Additional material examined. CHILE. Región de Arica y Parinacota. Prov. Arica, Lago Chungará, ribera occidental, $18^{\circ} 14^{\prime} \mathrm{S}, 69^{\circ} 10^{\prime} \mathrm{W}, 4500 \mathrm{~m}, 20-\mathrm{V}-1979$, Villagrán et al. 1271 (CONC); camino de Arica al Portezuelo de Chapiquiña, km 93, 3350 m, 8-II-1964, C. Marticorena et al. 29 (CONC); camino a Chapiquiña, Quebrada frente a la Central Hidroeléctrica, 3400 m, 8-V-1972, Ricardi et al. 279 (CONC); Prov. Parinacota, Putre, 3500 m, 23-XI-1955, Levi Heins 306 (CONC). Región de Tarapacá. Prov. El Tamarugal, camino de Cancosa al límite con Bolivia, km 2, 4000 m, 18-II-1964, Marticorena et al. 360 (CONC); $2 \mathrm{~km}$ al E de Chuzmisa,

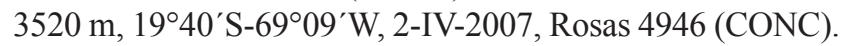
Región de Antofagasta. Prov. El Loa, Chiuchiu, en una huerta, 2560 m, 3-I-1950, Pfister s.n. (CONC 9364). Región de Coquimbo. Prov. Elqui, camino entre Embalse La Laguna y Campamento del Embalse, 30²' $\mathrm{S}, 70^{\circ} 02^{\prime} \mathrm{W}, 2900-3200 \mathrm{~m}$, 8-I-1981, Arroyo 81112-A(CONC). Región Metropolitana de Santiago. Prov. Santiago, Cerro San Cristóbal, 800 m, 14-V1954, Navas 678 (CONC). Región del Maule. Prov. Linares, a lo largo del río Achibueno, desde junta estero Las Ánimas a junta estero Riecillo, 30 $05^{\prime} \mathrm{S}, 71^{\circ} 10^{\prime} \mathrm{W}, 9-\mathrm{III}-1999$, Ruiz \& López 1231 (CONC). Región del Biobío. Prov Arauco, Isla Mocha, Laguna Hermosa, 300 m, 15-III-1971, Weldt \& Rodríguez 1149 (CONC); Prov. Nuble, Cobquecura, 20 m, 13-IV-1954, Ledezma 225 (CONC). 

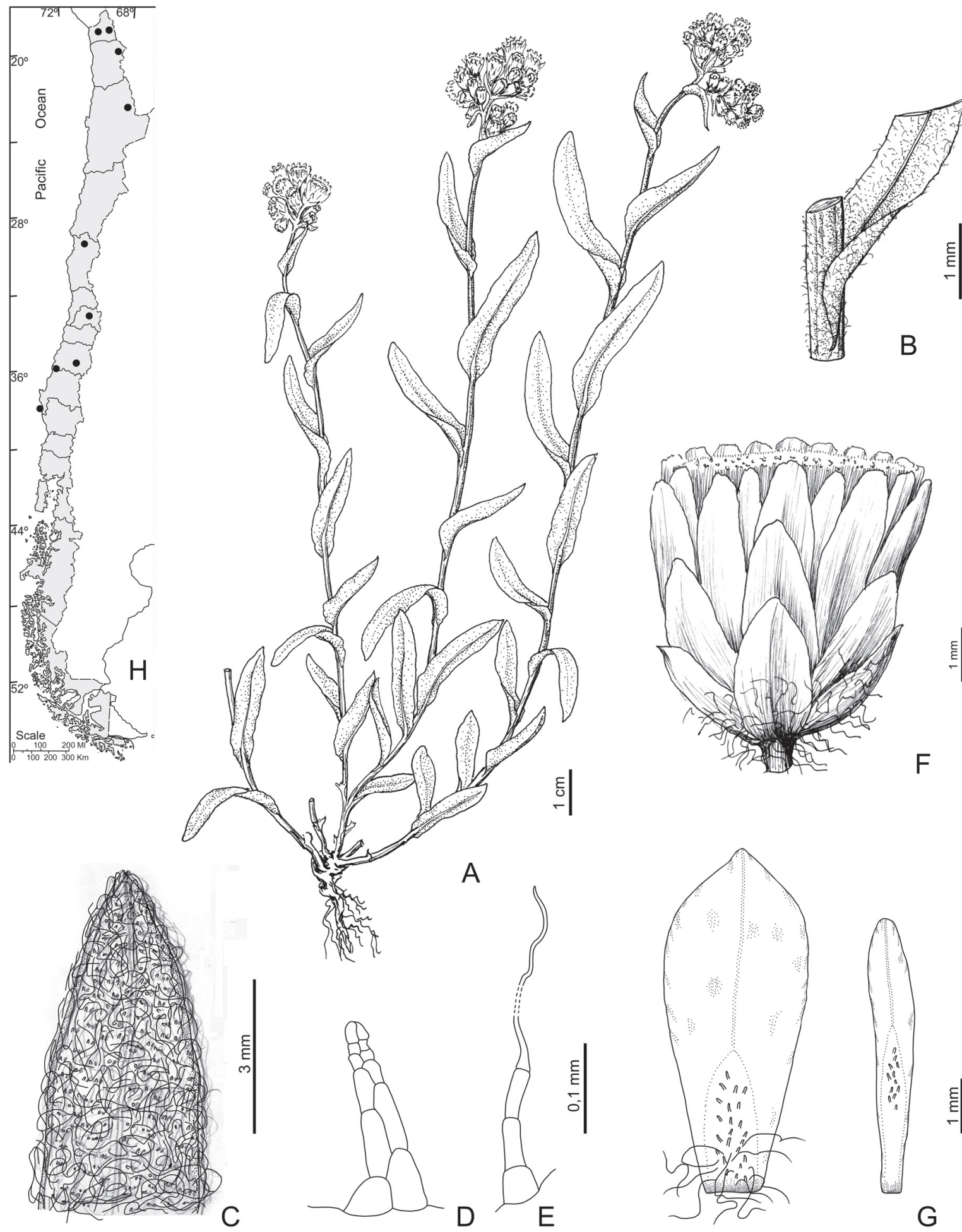

Figure 15. Pseudognaphalium tarapacanum. A, Habit; B, Leaf base; C, Leaf pubescence; D, E, Foliar trichomes; F, Capitulum; G, Phyllaries; H, Distribution [A, B, F, from Cabrera 1978; C-E, Marticorena et al. 360, CONC; G, Marticorena et al. 29, CONC].

Figura 15. Pseudognaphalium tarapacanum. A, Planta; B, Base de la hoja; C, Pubescencia de la hoja; D, E, Tricomas foliares; F, Capítulo; G, Filarios; H, Distribución [A, B, F, tomado de Cabrera 1978; C-E, Marticorena et al. 360, CONC; G, Marticorena et al. 29, CONC]. 
12. Pseudognaphalium viravira (Molina) Anderb., Opera Bot. 104: 148. 1991. Gnaphalium viravira Molina, Sag. Stor. Nat. Chili 149, 354. 1782. TYPE: Chile. Valparaíso 'La Vinna de la mar', IX-1830, C. L. G. Bertero 1823 (lectotype, here designated, CONC!; isolectotype, NY 00169526,= photo!). Fig. 16.

Gnaphalium montevidense Spreng., Syst. Veg. 3: 475. 1823. Pseudognaphalium montevidense (Spreng.) Anderb. Opera Bot. 104: 147. 1991, syn. nov. TYPE: Uruguay. Montevideo, Sello s.n. Herb. Sprengel 785 (holotype, P 704564, = photo!; isotipo, LP 001932! fragment ex P).

Gnaphalium coquimbense Phil., Linnaea 29: 5. 1858. Pseudognaphalium coquimbense (Phil.) Anderb., Opera Bot. 104: 147. 1991, syn. nov. TYPE: Chile. Coquimbo, La Serena, IX-1837, C. Gay 678 (holotype: SGO 64422!; isotype, B (probably destroyed ), = photo F15094 SI!).

Gnaphalium pratense Phil., Linnaea 33: 166. 1864. Pseudognaphalium pratense (Phil.) Anderb., Opera Bot. 104: 147. 1991, syn. nov. TYPE: Chile. Valdivia, Ranco, I-1860, R.A. Philippi s.n. (lectotype designated by Cabrera (1971: 112), SGO 71286!).

Gnaphalium illapelinum Phil., Linnaea 33: 164. 1864. Pseudognaphalium illapelinum (Phil.) Anderb., Opera Bot. 104: 147. 1991, syn. nov. TYPE: CHILE. Coquimbo, Illapel, XII-1862, L. Landbeck s.n. (lectotype, here designated SGO 64423!; isolectotypes, B (probably destroyed), = photo F15101!, LP 001923!, NY!, SGO 44966!).

Gnaphalium andicola Phil., Anales Univ. Chile 90: 17. 1895. Pseudognaphalium andicola (Phil.) C. Monti, N. Bayón \& S.E. Freire, J. Bot. Res. Inst. Texas 7(1): 196. 2013, syn. nov. TYPE: Chile. Santiago, Las Condes, L. Navarro s.n. (lectotype, designated by Monti et al. (2013: 196), SGO 64481!).

Gnaphalium longifolium Phil., Anales Univ. Chile 90: 13. 1895, syn. nov. TYPE: Chile. Valdivia, S. Juan, II-1882, Philippi s.n. (holotype, SGO 64446!; isotype, LP 001930!).

Gnaphalium subnudum Phil., Anales Univ. Chile 90: 15. 1895, syn. nov. TYPE: Chile. 'Habitat in andibus sed quo loco?' without collector (lectotype, here designated, SGO 64429 !).

Perennial herbs, 5-40(60) cm tall, multistemmed, stems erect or ascending, unbrached, more rarely branched at the upper part, whitish-woolly. Stem leaves 13-50 (60) × 1-5(10) $\mathrm{mm}$, linear or linear-ovate to linear-oblong, margin flat or occasionally undulate, apex acute to attenuate, base clasping and decurrent 2-7(10) mm long; basal leaves commonly approximate, $23-50 \times 3-6(10) \mathrm{mm}$, linear-obovate, apex obtuse, base long-attenuate; concolorous, whitish-woolly to arachnoid-glandulose on both surfaces, trichomes of 2 types, long eseptate eglandular, 3-6-celular and very few short biseriate glandular trichomes hidden under the wool. Capitula numerous in dense terminal clusters, sometimes arrranged in corymbs. Involucre campanulate, 3-5 × 3-5 $\mathrm{mm}$. Phyllaries 3-4-seriate, lamina yellowish or light brown to whitish, hyaline and shiny; outer phyllaries 2.8$4.2 \times 1.4-2 \mathrm{~mm}$, obovate, apex obtuse or acute; inner phyllaries 3-4.3 $\times 1-1.1 \mathrm{~mm}$, linear-elliptic, apex acute or subobtuse. Pistillate florets, (82-88)115-130, corolla yellow, filiform, (2.8)3.7-4 mm long. Bisexual florets 5-16, corolla yellow, tubular, (2.7)3.3-3.7 mm long. Achenes 0.4$0.5 \mathrm{~mm}$ long, glabrous with smooth or papillose epidermis or pilose with short oblong duplex hairs, sometimes with papillose epidermis and few short oblong duplex hairs. Pappus bristles (2.2)3.2-4 mm long.

Pseudognaphalium viravira is treated here as a polymorphic species, with plants ranging from few centimetres to near half meter tall, upper leaves varying from linear to linearobovate, acute to attenuate at the apex, with margins flat or rarely obscurely undulate, usually woolly to more rarely arachnoid-glandulose, and glabrous to pilose achenes.

Phenology. Flowering from August to March.

Vernacular name. 'Nido de diuca', 'vira-vira'.

Distribution And habitat. Chile (Tarapacá, Atacama, Coquimbo, Valparaíso, Metropolitana, Libertador General Bernardo O'Higgins, Maule, Biobío, Araucanía, Los Lagos, Aysén del General Carlos Ibáñez del Campo, and probably Magallanes y de la Antártica Chilena) and central-southern Argentina (Buenos Aires, Chubut, Jujuy, La Rioja, Mendoza, Neuquén, Río Negro, Salta, San Juan, Santa Cruz, Tierra del Fuego), growing on dry soils or in forest in shady places; from sea level to $3200 \mathrm{~m}$.

Relationships. Pseudognaphalium viravira can be distinguished by its woolly mutistemmed plants, unbranched stems, basal leaves usually approximate, upper leaves usually remote, and capitula in dense terminal clusters (arranged in corymbs when plants fully developed). Pseudognaphalium viravira is closely related to the cosmopolitan species Pseudognaphalium luteoalbum (L.) Hilliard \& B.L. Burtt. Both species have woolly leaves on both surfaces; approximate basal leaves with linear-spathulate, apically obtuse leaf blade; remote stem leaves with oblong-linear, apically acute, occasionally undulate margin leaf blade, and capitula arranged in dense terminal clusters with involucre 3-5 $\mathrm{mm}$ high. P. luteoalbum differs from this species by its annual plants (vs. perennial in P. viravira) and yellowish 

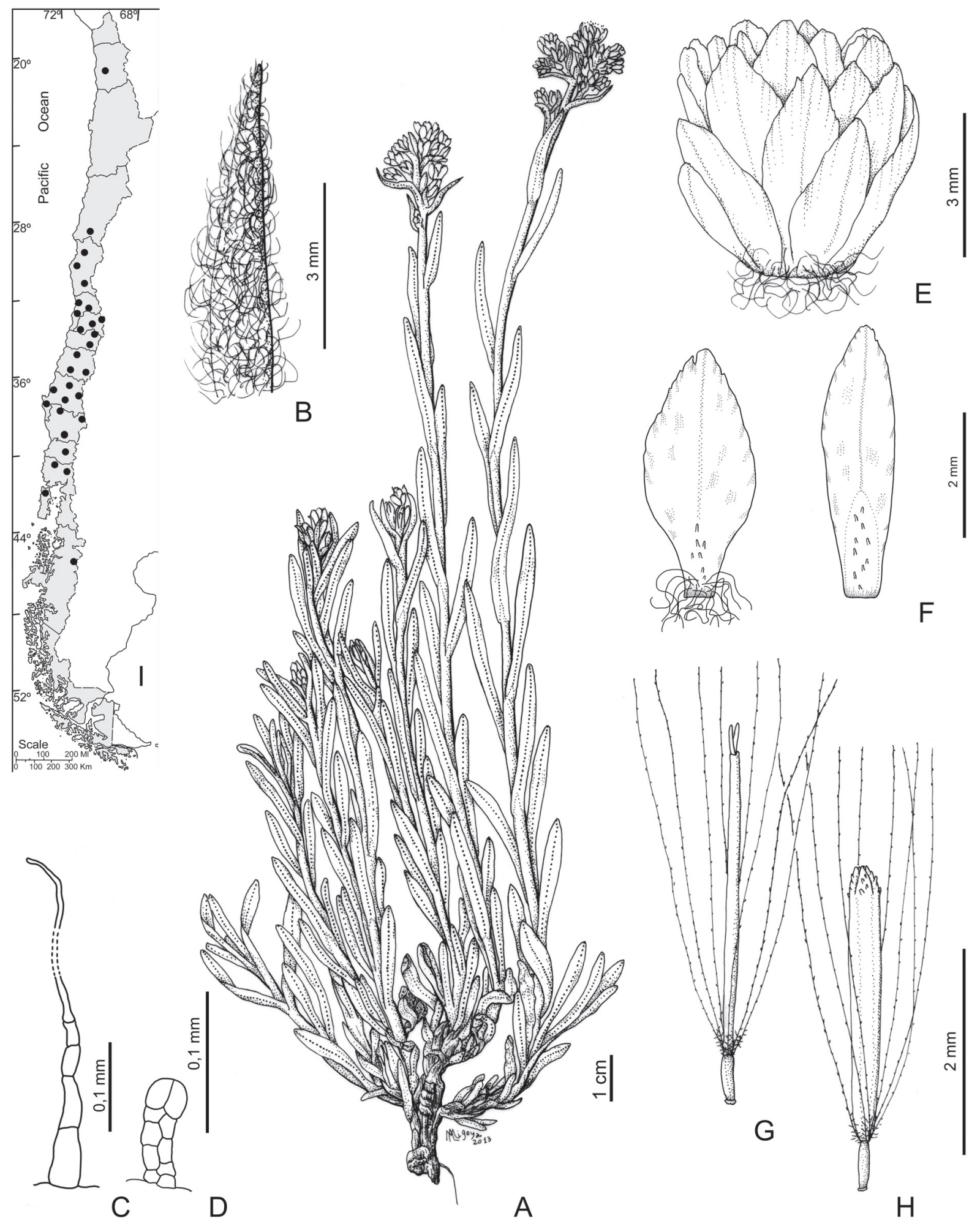

Figure 16. Pseudognaphalium viravira. A, Habit; B, Leaf pubescence; C, D, Foliar trichomes; E, Capitulum; F, Phyllaries; G, Pistillate floret; H, Bisexual floret. I, Distribution [A-H, Gunckel 39466, CONC].

Figura 16. Pseudognaphalium viravira. A, Planta; B, Pubescencia de la hoja; C, D, Tricomas foliares; E, Capítulo; F, Filarios; G, Flor pistilada; H, Flor perfecta; I, Distribución [A-H, Gunckel 39466, CONC]. 
florets, usually reddish at apex (vs. whitish in P. viravira). Although P. luteoalbum was mentioned to RME (Rémy 1849, Iboda 2014), we have not seen specimens of this species.

\section{OBSERVATIONS}

1. The protologue of Gnaphalium viravira did not include type collection. Later, Candolle (1838: 224), mentioned for G. viravira, two specimens collected by Bertero, i.e. 'Bert. n. 1032 et 1823 ' from Quillota and Valparaíso, respectively. We located the specimen 'Bertero1823' at CONC, and at NY a sheet with two specimens mounted on it, one with double collection numbers Bertero 309 \& 1032 (NY 00169525), 'In pascuis petrosis calidis Quillota, et ad sepes secus vias prope La Quinta, Chili. Octbr. Nvbr. 1829', and the other Bertero 1823 (NY 169526), 'la Vinna de la mar. Valparaiso. Chili. Spbr. 1830'. A third sheet of Bertero 309 \& 1032 was located at MO (MO 5448098, = photo!). We propose to select as lectotype the specimen 'Bertero1823' kept at CONC which presents the most complete plant, and to exclude from type material the specimen Bertero $309 \&$ 1032 (NY 169525, MO 5448098) because of its ambiguous information.

2. After examining the type materials, Gnaphalium montevidense, G. pratense, and G. andicola are proposed here as new synonyms of G. viravira because there is no diagnostic character to separate them as distinct taxa. All of these are multistemmed whitish-woolly plants, with approximate basal leaves and remote stem leaves that are linear to linear-obovate and apically acute, and capitula arranged in dense terminal clusters. In addition, the differential character mentioned in the protologue of Gnaphalium montevidense, i.e., stem leaves clasping, is a variable feature throughout the range of G. viravira.

3. Gnaphalium andicola was placed into synonymy of Pseudognaphalium cheiranthifolium (Hind 2011). According to the protologue of G. andicola and Gnaphalium cheiranthifolium, they have leaves 'lanceolato-linearibus longe acuminatis' and 'foliis lineari-lanceolatis acutis', respectively. However, examination of the original material of both species at SGO and P, respectively reveals that the leaves of Gnaphalium andicola are linear to narrowly linearobovate, apically acute (vs. lancelolate to linear-lanceolate, apically attenuate in G. cheiranthifolium). In addition, Gnaphalium andicola differs from P. cheitanthifolium by it multistemmed plants (vs. solitary stems, rarely 2 or 3, in $P$. cheiranthifolium), and its capitula usually in solitary terminal clusters (vs. capitula in cluster arranged in coryms in P. cheiranthifolium).

4. After examining the type materials, Gnaphalium coquimbense is proposed here as a new synonym of $G$. viravira because there is no diagnostic character that differentiates the former from the latter. Both are multistemmed whitish-woolly plants, with linear spathulate basal leaves and remote stem leaves that are apically acuminate, shortly decurrent at the base. In addition, the differential characters mentioned in the protologue of $G$. coquimbense, i.e., congested inflorescences and yellowish phyllaries, are variable features throughout the range of $G$. viravira.

5. The protologue of Gnaphalium illapelinum mentions the collection 'Prope Illapel, Lanbeck lectum'. We located at SGO and LP the collection 'Illapel, December 1862', SGO 44966, 64423; LP 001923, and the colection 'Illapel, prov. Coquimbo' at NY. Even if these four specimens are in accordance with the protologue, we selected as the lectotype of Gnaphalium illapelinum the specimen SGO 64423, which presents the most complete plant and has Cabrera's annotation 'Typus'.

6. After examining the type materials, Gnaphalium illapelinum is proposed here as a new synonym of $G$. viravira because there is no diagnostic character that differentiates the former from the latter. Both are multistemmed whitishwoolly plants, with approximate basal leaves and remote stem leaves that are apically attenuate, and capitula arranged in dense terminal clusters. In addition, the differential character mentioned in the protologue of G. illapelinum, i.e., arachnoid glandulose leaves, is a variable feature throughout the range of G.viravira.

7. According to the protologue, Gnaphalium subnudum was based on three specimens without locality, i.e. 'tenemos tres ejemplares, de los cuales el mayor ...' . We found at SGO a sheet with two specimens mounted on it, which has Philippi's annotation on the right-hand side 'Gnaphalium subnudum'. This specimen which is in accordance with the protologue of Gnaphalium subnudum, is here designated as the lectotype.

8. Gnaphalium longifolium Phil. and G. subnudum Phil., which were previously placed into synonymy of $G$. pratense Phil. and G. montevidense, respectively (Cabrera 1971), are here considered as synonyms of Pseudognaphalium viravira.

Additional material examined. CHILE. Región de Tarapacá. Prov. El Tamarugal, Termas de Mamiña, 2-4 km antes del pueblo, $2700 \mathrm{~m}, 18-\mathrm{X}-1965$, Ricardi et al. 1348 (CONC); Mamiña, 2750 m, IX-1956, Richter s.n. (CONC 116230). Región de Atacama. Prov. Huasco, Río Laguna Grande, entre Quebrada Candelilla y el extremo oeste de la Laguna Grande, 2800-3100 m, 2-I-1983, C. Marticorena et al. 83413 (CONC); S/Prov., Estancia Manflas, $1450 \mathrm{~m}$, 2-XI-1956, Ricardi \& C. Marticorena 3747 (CONC). 
Región de Coquimbo. Prov. Choapa, Illapel, 19-IX-1926, Barros 1466 (CONC); ídem, 310 m, VIII-1971, Zöllner 5133 (CONC); ídem, s/fecha, Philippi s.n. (NY); Los Vilos near the coast, 30-X-1976, Zöllner 9914 (CONC, MO); Valle Ojotas, Potrero Largo, 3200 m, 8-II-1984, Zöllner 12142 (SI); Cordillera de Combarbalá, Potrero Grande, Ramadilla, 31 $18 \mathrm{~S}, 70^{\circ} 50 \mathrm{~W}, 2600 \mathrm{~m}, 6-\mathrm{I}-1963$, Jiles 4428 (CONC); Quilimari, 5 m, 9-IX-1982, Montero 12281 (CONC); Prov. Elqui, entre La Serena y la Cuesta de Las Chilcas, orillas de la Carretera, 20-IX-1974, Mahu 10213 (LP); Punta de Teatinos, II-1950, Chang 20965 (CONC); La Serena, 15 m, IX-1926, Barros 2424 (CONC); ídem, I-1928, Barros 21755 (CONC); ídem, IX-1929, Barros 2840 (CONC); Valle del Elqui, El Molle, road leading E from village, along the $\mathrm{N}$ side of the river, $29^{\circ} 58^{\prime} 51$ " S, 7056' 17 'W, 365 m, 7-XI-2006, Tepe et al. 1840 (CONC); Coquimbo, besides the Panamerican near Teniente Bridge, 10-IX-1987, Zöllner 13652 (MO); Prov. Limarí, Ovalle, IX1926, Barros 2417 (CONC); Ovalle, Zorrilla, 3050'S, $71^{\circ} 30^{\prime} \mathrm{W}, 350 \mathrm{~m}, 26-\mathrm{IX}-1946$, Jiles 910 (CONC); ́́dem, 17IX-1950, Jiles 1818 (CONC, LP); Ovalle, Parque Nacional Fray Jorge, parte baja, $30^{\circ} 40^{\prime} \mathrm{S}, 71^{\circ} 33^{\prime} \mathrm{W}, 300 \mathrm{~m}, 5-\mathrm{XI}-$ 1974, C. Marticorena et al. 421 (CONC); Ovalle, Carretera Panamericana, $5 \mathrm{~km}$ al S de Socos, 28-X-1965, Ricardi et al. 1551 (CONC); Cordillera de Ovalle, Cerro Loica, $31^{\circ} 02^{\prime} \mathrm{S}, 72^{\circ} 42^{\prime} \mathrm{W}, 2000 \mathrm{~m}, 18-\mathrm{XII}-1965$, Jiles 4735 (CONC); Hurtado Valley, 16-IX-1986, Zöllner 13111 (MO). Región de Valparaíso. Aconcagua, Pichidangui, 470 m, 18II-1962, Mahu s.n. (LP); Prov. Marga Marga, Limache, 31V-1924, A.J.H. 680 (CONC 89681); ídem, 18-IX-1927, Looser 203 (SI); Villa Alemana, 21-IX-1937, Barros 2301 (LP, SI); Valle de Marga-Marga, $40 \mathrm{~km}$ al E de Valparaíso, IX-1916, Jaffuel 896 (GH); Marga-Marga, 150 m, IX-1910, Jaffuel 899 (CONC); Prov. Quillota, Cuesta de Melón, 13IX-1957, Ricardi \& C. Marticorena 4222 (CONC); Prov. Petorca, Papudo, 28-X-1974, Zöllner 7936 (CONC); Rt. 5 between tunnel and La Ligua, $32^{\circ} 33^{\prime} 25^{\prime}$ 'S, $71^{\circ} 15^{\prime} 54^{\prime \prime} \mathrm{W}$, 110 m, 3-XI-2006, Tepe et al. 1689 (CONC); Prov. San Felipe de Aconcagua, Carretera Panamericana, Bajada de El Melón, 13-IX-1957, Cabrera 12523 (LP); Prov. Valparaíso, Viña del Mar, 24-IX-1921, Behn 20879 (CONC); Jardín Botánico Nacional de Viña del Mar, 10-V-1956, Cabrera 12215 (LP); ídem, Sendero Marianne North, 332'15”'S, 71²9'38"W, 21-I-2005, Novoa s.n. (CONC 165314); Quintero, Los Molles, $32^{\circ} 46^{\prime} \mathrm{S}, 71^{\circ} 32^{\prime} \mathrm{W}, \mathrm{XII}-1953$,

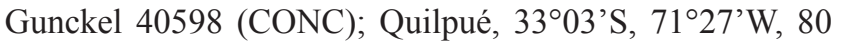
m, 13-XI-1969, Schlegel 3144 (CONC); Chorrillos, 26-XII1944, Boelcke 363 (SI); Concón, 15 m, XII-1951, Gunckel 23453 (CONC); Tranque Pitama, 250 m, 15-XI-2010, Rabanales s.n. (CONC 173012). Región Metropolitana de Santiago. Prov. Chacabuco, Batuco, 480 m, 25-IX-1951, Gunckel 22760 (CONC); Prov. Cordillera, Clarillo, 800 m, Gunckel 39466 (CONC); Prov. Maipo, Laguna de Aculeo, 350 m, 11-X-1942, Pisano et al. 1567 (CONC); Prov.
Santiago, Quebrada de Macul, XI-1965, Muñoz 48718 (CONC); Cerro de Renca, 750 m, XI-1950, Gunckel 22280 (CONC); Subida al Cerro Caléu, $33^{\circ} 00^{\prime} \mathrm{S}, 70^{\circ} 57^{\prime} \mathrm{W}, 800 \mathrm{~m}$, 13-XI-1960, Schlegel 3125 (CONC); San José de Maipo, Cajón de Morales, entre Panimávidas y la laguna, 2100 m, 25-I-2002, Teillier \& Márquez 5315 (CONC); Monumento

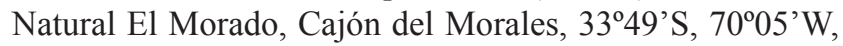
2300 m, 29-XII-1990, Teillier \& González 2301 (CONC, MO); Cajón del Río Maipo, Quebrada El Canelo, 12-XII1973, Mahu 9795 (LP); Cajón del Maipú, Potrero Grande, 2800 m, 2-I-1967, Zöllner 1770 (LP); San José de Maipo, Cajón del río Morales, 17-I-1989, Saavedra \& Pauchard 111 (CONC); Alhué, Monte Santillana, 2-I-1939, Barros 2285 (LP, SI); Lo Valdés (Baños Colina), 2500 m, 2-I-1968, Zöllner 3031 (CONC); Lo Valdés, 2300 m, 7-III-1953, Ricardi 2448 (CONC); ídem, en las cercanías del Refugio alemán, Quebrada Valdés, 2000 m, 20-XI-1940, Garaventa 5681 (CONC); entre Lo Valdés y las Yeseras, 2450 m, 11-II1963, Ricardi et al. 834 (CONC); Río Colorado, $2500 \mathrm{~m}$, I-1950, Moreno 25689 (CONC); mounts above Río Colorado, 4000 ft, 21-I-1902, Hastings 418 (NY); Pirque, Cerro Blanco (Puente Alto), 11-V-1970, Mahu 4885 (LP); Quebrada de Ramón, 33²6'S, 70³0'W, 1200-1600 m, 19XI-2000, Tomé 130 (CONC); Fundo Valencia, Monte Águila, 1/3-XI-1939, Looser 3990 (LP); Prov. Talagante, Peñaflor, 400 m, XI-1965, Arellano 48720 (CONC). Región del Libertador General Bernardo O'Higgins. Prov. Colchagua, San Fernando, 1700 m, 3-I-1959, Montero 6056 (CONC); San Fernando, Vegas Del Flaco, 1800 m, 7-II1955, Ricardi 3157 (CONC); Termas del Flaco, 3456'S, $70^{\circ} 25^{\prime} \mathrm{W}, 1950$ m, 3-II-1989, Niemeyer \& Fernández 8908 (CONC); Río Tinguiririca, San Fernando, 300 m, 29-XII1950, Ricardi s.n. (CONC 9874); Prov. Cachapoal, Palmar

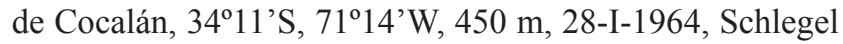
4949 (CONC). Región del Maule. Prov. Curicó, Cajón del Lontué, I-1884, Philippi s.n. (sintipo de G. andicola, SGO 064385); Lolol, 17-IX-1937, Barros 184 (LP); 9 km al E de Los Queñes, 3501'32”S, 7042'53”'W, 815 m, 13-XI-2006, Tepe 2003 (CONC); Cordillera del Potrero Grande, 30-I1928, Barros 12 (LP); Peteroa, I-1933, Grandjot s.n. (SI); Peteroa, 2800 m, I-1933, C. \& G. Grandjot s.n. (SI 141479); Cordillera de El Planchón, 17-II-1939, Barros 2304 (LP, SI); camino de Laguna de Teno a la junta con el camino internacional a Paso Vergara, $2250 \mathrm{~m}, 10-\mathrm{III}-1967, \mathrm{C}$. Marticorena \& Matthei 973 (CONC), $2300 \mathrm{~m}, \mathrm{C}$. Marticorena \& Matthei 967 (CONC); Prov. Linares, Cauquenes, Fundo Porvenir, 250 m, 29-X-1975, Rodríguez 661 (CONC); Reserva Nacional Los Bellotos del Melado, quebrada, 1415 m, 4-I-2000, Humaña et al. 20102 (CONC); Reserva Nacional Los Bellotos del Melado, 1374 m, Gardner et al. 295 (CONC); Altos de Vilches, camino a Linares, fundo El Castillo, entrando por Copihue hacia el este, 945 m, 6-III-1999, Ruiz \& López 1018 (CONC); a lo largo del río Achibueno, desde junta estero Las Ánimas a 
junta estero Riecillo, 36 $06^{\circ}$ 'S, $71^{\circ} 10^{\prime} \mathrm{W}$, 9-III-1999, Ruiz \& López 1184 (CONC); Prov. Talca, Laguna del Maule, Talca, 2200 m, I-1943, Behn s.n. (CONC 4264, 20878); Maule, 8-II-1892, Kuntze s.n. (NY); Prov. Talca, Constitución, Quivolgo, 26-XI-1958, A. Barnier 427 (CONC); Curepto, I-1926, Barros 477 (CONC); camino de Talca hacia Putú, fundo El Trapiche, $120 \mathrm{~m}$, 4-I-1964, Matte 745 (CONC); Altos de Vilches, camino a Laguna El Alto, 2000 m, 29-I2000, Finot \& López 1804 (CONC); Laguna de la Invernada, orilla oeste, $35^{\circ} 43^{\prime} \mathrm{S}-70^{\circ} 46^{\prime} \mathrm{W}, 1300 \mathrm{~m}, 11-\mathrm{IV}-2000$, Finot \& López s.n. (CONC); Comuna de San Clemente, Paso Pehuenche, $1750 \mathrm{~m}$, I-2005, Luebert \& Teillier 2232 (CONC). Región del Biobío. Prov. Arauco, Playa Punta Morguilla, 37 $42^{\prime} \mathrm{S}, 7^{\circ} 38^{\prime} \mathrm{W}$, 6-II-2001, Parra \& Torres 431 (CONC); Prov. Biobío, Panamericana a Pangal del Laja, $\mathrm{km} \mathrm{10,} 150 \mathrm{~m}, 37^{\circ} 11^{\prime} \mathrm{S}, 7^{\circ} 17^{\prime} \mathrm{W}, 29-\mathrm{XI}-1976$, Marticorena \& Rodríguez 8430 (CONC); Laguna de la Laja, 21-I-1969, Cabrera 19677 (LP); Laguna del Laja, faldeos Sierra Velluda, 15-II-1960, Ricardi \& Marticorena 5150 (CONC); Laguna del Laja, Los Barros, 22-I-1969, Ricardi \& C. Marticorena 5766 (CONC); Laguna del Laja, Los Barros, sector Mallín Florido, 1458 m, 23-I-2001, Baeza et al. 3110 (CONC); faldeos del volcán Antuco, frente a la Laguna del Laja, 21-I-1969, Ricardi \& C. Marticorena 5721 (CONC); Parque Nacional Laguna del Laja, camino Los Barros a Pichachén, 1470 m, 23-I-2001, Baeza et al. 3074 (CONC); entre Carretera Longitudinal y Pangal del Laja, km 10, $37^{\circ} 11^{\prime} \mathrm{S}, 7^{\circ} 17^{\prime} \mathrm{W}, 29-\mathrm{XI}-1976$, C. Marticorena \& Rodríguez 8417 (CONC); El Roble, 2-II1929, Barros 22611 (CONC); Prov. Concepción, fundo Bolslal, 5-XII-1933, Belisario 849 (CONC); camino a Coronel, km 15, 7-XII-1962, Gleisner s.n. (CONC 89714); camino de Rere a Yumbel, 6-I-1959, Marticorena et al. 43 (CONC); Pichaco, Hualqui, 12-VIII-1936, Junge s.n. (CONC 5891); San Pedro, 1-XI-1943, Barros 3139 (SI); Dunas de San Vicente, 24-X-1934, Junge 6285 (CONC); Florida, Fundo Colico, 11-IX-1976, Oehrens s.n. (CONC 89742); camino de Hualqui a Rere, cerca de Gomero, 220 m, 5-I-1959, C. Marticorena et al. s.n. (CONC 25211); Prov. Concepción/Ñuble, camino Concepción-Bulnes, $70 \mathrm{~m}$, 6-XII-1944, Pfister s.n. (CONC 4949); Prov. Ñuble. Baños de Chillán, 28-XII-1986, Zöllner 13147 (MO); Reserva Nacional Nuble, al Este de la avanzada de Cuatro Juntas, junto al camino hacia la frontera, $1500 \mathrm{~m}, 7-\mathrm{II}-2008$, Teneb 551 (CONC); Reserva Nacional Ñuble, al Este de la avanzada de Cuatro Juntas, junto al camino cerca de la pista del gasoducto, $1500 \mathrm{~m}$, 7-II-2008, Teneb 569 (CONC); camino a termas de Chillán, Gruta Los Pangues, 1200 m, 7-XII-1992, Rodríguez \& C. Marticorena 3053 (CONC); Ñuble, Termas de Chillán, 1800 m, Cabrera 3636 (LP); Termas de Chillán, 1750 m, 14-I-1967, Schlegel 5863 (CONC); Nevados de Chillán, Ruinas del Refugio Walldorf, 1940 m, 13-I-2009, Pfanzelt 173 (CONC). S/Prov., El Roble, 3-II-1929, Barros 18 (LP); Chancho Co, 15-II-1947, de Barba 2040 (LP); El Abanico, III-1949, Pfister s.n. (CONC 8864). Región de la Araucanía. Prov. Cautín, Parque Nacional Villarrica, $1070 \mathrm{~m}$, 12-I-1993, Finckh 552 (CONC); Cunco, 13-I-1931, Barros 44 (LP); Laguna Conguillío, 1150 m, 20-I-1976, C. Marticorena et al. 779

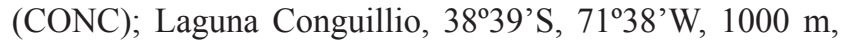
II-1963, Gleisner 201 (CONC); Prov. Malleco, Angol, Lealtad, 6-XI-1958, Gunckel 374 (CONC); Angol, Deuco, 8-XII-1960, Montero 6396 (CONC); Angol, 80 m, 4-I-1923, Barros 3370 (CONC); camino entre Lonquimay y cordillera de las Raíces, 4-I-1947, Pfister s.n. (CONC 7201); Lonquimay, 2-III-1939, Burkart 9491 (SI); camino entre Lonquimay y Liucura km 31, 1000 m, 10-II-1960, Ricardi \& C. Marticorena 5052 (CONC); camino de Icalma a Liucura, 16-I-1947, Pfister s.n. (CONC 7396); valle de Lonquimay, 1000 m, 7-I-1947, Pfister s.n. (CONC 7273); Malleco, Termas de Río Blanco, 1-I-1948, Pfister s.n. (CONC 7868); Curacautin, Termas Río Blanco, 28-I-1938, Montero 3667 (CONC 78523, 92599); a orillas del estero Lancú, en la confluencia con el río Lolco, 950 m, 9-I-1977, C. Marticorena et al. 1351 (CONC); Mininco, 20-I-1953, 190 m, Gunckel 819 (CONC); camino al Parque Nacional Nahuelbuta, $1 \mathrm{~km}$ más arriba de los Lleulles, 3746'S, 7247’W, 800 m, 16-I-1986, R. Rodríguez \& Pacheco 1912 (CONC). Región de los Lagos. Prov. Chiloé, Pudeto, Purranque, 9-II-1961, C. Marticorena 1591 (CONC); Prov. Llanquihue, Petrohué, Lago Todos Los Santos, 210 m, 24II-1944, Pfister 4277 (CONC). Región de Aysén del General Carlos Ibáñez del Campo. Prov. Coyhaique, Coyhaique, 16I-1946, Barros 6076 (LP); S/ Prov., Valle Ibáñez, 740 m, 25-I-1939, Von Rentzell 6138 (SI 022629); Est. Guido, 17I-1952, Pfister \& Ricardi s.n. (CONC 12181). Región de Magallanes y Antártica Chilena. Sin loc. determinada, 1838-1842, Herbarium of the U.S. South Pacific Exploring Expedition under the command of Capt. Wilkes, U. S. N. (NY, PH 01084668).

\section{NAMES OF DUBIOUS IDENTITY}

Gnaphalium puberulum DC., Prodr. 6: 224. 1838. TYPES: 'in Chili ad montem la Leona specim. anthesi incipiente legit cl. Bertero (herb. n. 299!) et in Brasilia si Gn. decurrens Schrank pl. Mont. t. 84 (non Yves) huc ex icone ritè referendum?'.

According to the protologue two syntypes were cited, one from Chile (Bertero 299) and other from 'Brasilia'. We found at $\mathrm{P}$ (P00704533, = phot!) the collection from Chile 'In rupestribus herbidis Montis La Leona. Rancagua. Chili. Bertero herb. N²99 / 1818, 8bre.' (year 1833, in the sheet at G-DC, not seen), which is in accordance in collector, number and locality with the protologue but, as was pointed out by Cabrera in this specimen, through an annotation on the specimen's sheet, its characters differ (probably refering 
to the pubescence) from the protologue. It says 'caule ... etomentosis pube brevi subglandulosa puberulo-hirtellis' ... 'Pubes fortè viscosa, sed á G. viscoso...'vs. pubescence long lanuginose (probably with short glandular trichomes hidden under the wool) on stems and leaves in the material. Gnaphalium puberulum DC. resembles Pseudognaphalium viravira in having approximate basal leaves and remote stem leaves that are apically attenuate, and capitula arranged in dense terminal clusters. However since we were unable to see the original material and we have seen no other material which can be assigned to this species, the identity of $G$. puberulum is not clear.

Gnaphalium chilense Spreng., Syst. Veg. 3: 480. 1826. Gnaphalium sprengelii Hook. \& Arn., Bot Beechey Voy.: 150. 1833, nom. superfl. TYPE. 'Chili. Chamisso. Peru?' (not seen).

Gnaphalium sprengelii Hook. et Arn. (Bot Beechey Voy.: 150. 1833) is a nomen supefluous because this name was created to rename Sprengel's Gnaphalium chilense (Syst. Veg. 3: 480. 1826), since Hooker and Arnott considered that the species was originally native from California and not from Chile. Unfortunately, type collection of Gnaphalium chilense Spreng., 'Chili. Chamisso. Peru?', was not located at $\mathrm{P}$, where the original herbarium of Sprengel is probably deposited. For this reason, until the type of Sprengel's name is located or any materials can be provided, the identity of G. chilense is uncertain.

\section{EXCLUDED SPECIES}

Gnaphalium oliganthum Phil., Linnaea 33: 167. 1864. TYPE: 'In collibus aridis prope Catemu prov. Aconcagua legi.' = Gamochaeta oligantha (Phil.) L.E. Navas (Freire \& Iharlegui 2008).

Gnaphalium pedunculatum (Poepp. et Endl.) Benth. et Hook. ex Klatt, Linnaea 42: 142. 1878. Lucilia pedunculata Poepp. et Endl., Nov. gen et sp. 3: 51.1832. Type: CHILE. Andes de San Jago, Jan 1829, Poeppig s.n. (W not seen, digital image!, photo SI!) = Chevreulia pusilla DC.

\section{OBSERVATION}

According to the protologue and digital image of Lucilia pedunculata it corresponds to Chevreulia pusilla by having capitula longly pedunculate.

Gnaphalium ramosum Phil., Linnaea 33: 164. 1864, nom. illeg., non Lam. 1779 nec Sch. Bip. 1845. TYPE: Chile. Aconcagua. Concumen, I-1863, C.L. Landbeck s.n. (lectotype, here designated, SGO 64485!; isolectotype LP 001942!) = Gamochaeta
OBSERVATIONS

After examining the type material, we propose to exclude Gnaphalium ramosum from Gnaphalium since it lacks the diagnostic character of the genus in having bristles pappus fused at the base instead of bristles pappus with bases cohering by patent cilia.

Muñoz Pizarro (1960: 143) mentioned 'Hay un ejemplar colectado cerca de Concumén, en la provincia de Aconcagua (67787)' of Gnaphalium ramosum Phil.We selected as lectotype SGO 64485 since the sheet SGO 67787 was not located.

Gnaphalium suffruticosum Phil., Linnaea 33: 165. 1864. TYPES: 'Prope Illapel et Choapa invenit orn. Landbeck' = Gamochaeta suffruticosa (Phil.) Anderb. (Freire \& Iharlegui 2008).

\section{ACKNOWLEDGEMENTS}

We thank the reviewers for useful comments on a first draft of the manuscript. Appreciation is expressed to the directors and curators of the herbaria for the loan of specimens and copies of the types that made this study possible. Special thanks are due to Werner Greuter (B) who kindly helped us with nomenclatural aspects of Pseudognaphalium cabrerae. We would like to express our sincere appreciation to Matthias Svojtka (W), for providing literature not available in our countries. We thank María Alejandra Migoya for the illustrations of Pseudognaphalium aldunateoides, P. cabrerae, P. cymatoides, P. gayanum, $P$. landbeckii, $P$. remyanum, $P$. viravira, and drawing the maps. Financial support (PIP 112-200801-02196) was provided by Consejo Nacional de Investigaciones Científicas y Técnicas (CONICET), Argentina, the Chilean Comisión Nacional de Ciencia y Tecnología (CONICYT: Fondecyt 111085016/120448), and Universidad Nacional de La Plata, Programa de Incentivos, Secretaría de Políticas Universitarias, Ministerio de Educación, Argentina.

\section{LITERATURE CITED}

AnderberG, A.A. 1991. Taxonomy and phylogeny of the tribe Gnaphalieae (Asteraceae). Opera Botanica 104: 1-195.

Ballard, H.E., D.S. Feller Jr. \& G.L. Nesom. 2004. Cliff Cudweed at specific rank in Pseudognaphalium (Asteraceae: Gnaphalieae). Sida 21: 777-780.

Bayer, R.J., I. Breitwieser, J. Ward \& C. Puttock. [2006] 2007. Tribe-Gnaphalieae. In: J.W. Kadereit \& C. Jeffrey (eds.), (K. Kubitzki - series editor), The families and genera of vascular plants, flowering plants - Eudicots: Asterales, vol. 8, pp. 246-283. Berlin Springer-Verlag.

BAYón, N.D. \& D.A. Giuliano. 2013. Pseudognaphalium fastigiatum (Compositae: Gnaphalieae) es el nombre 
correcto para $P$. cabrerae. Boletín de la Sociedad Argentina de Botánica 48(3-4): 599-601.

Cabrera, A.L. 1963. Compositae: Gnaphalium. In: A.L. Cabrera (ed.), Flora de la Provincia de Buenos Aires 6(4), Colecc. Cient. INTA, Buenos Aires, pp.161-166.

Cabrera, A.L. 1971. Compositae: Gnaphalium. In: M.N. Correa (ed.), Flora Patagónica 8(7), Colecc. Cient. INTA, Buenos Aires, pp: 109-117.

Cabrera, A.L. 1974. Compositae: Gnaphalium. In: A. Burkart (ed.), Flora Ilustrada de Entre Ríos (Argentina) 6(6), Colecc. Cient. INTA, Buenos Aires, pp. 315-317.

Cabrera, A.L. 1978. Compositae: Gnaphalium. In: A.L. Cabrera (ed.), Flora de la Provincia de Jujuy, República Argentina 13(10), Colecc. Cient. INTA, Buenos Aires, pp. 275-288.

Candolle, A.P. De. 1838. Prodromus Systematis Naturalis Regni Vegetabilis 6. Paris, Strasbourg, London, 687 pp.

Chen, Y., S. Zhu \& R.J. Bayer. 2011. Tribe Gnaphalieae. In: Z.Y. Wu, P.H. Raven \& D.Y. Hong (eds.), Flora of China vols. 20-21 (Asteraceae). pp. 774-818. Science Press, Beijing \& Missouri Botanical Garden Press, St. Louis.

Deble, L.P. \& J.N.C. Marchiori. 2006. Sinopse de Pseudognaphalium Kirp. (Asteraceae-Gnaphalieae) no Brasil. Balduinia 9: 13-16.

Dillon, M.O. \& A. Sagástegui-Alva. 1991a. Sinopsis de los géneros de Gnaphaliinae (Asteraceae-Inuleae) de Sudamérica. Arnaldoa 1: 5-91.

Dillon, M.O. \& A. Sagástegui-Alva. 1991b. Gnaphalium. In: J.F. Macbride \& collab. (eds.), Flora of Peru, Family Asteraceae: Part V, pp. 32-41. Fieldiana: Botany n.s. 26 (1422).

Dizeo de Strittmatter, C. 1973. Nueva técnica de diafanización. Boletín de la Sociedad Argentina de Botánica 15: 126-129.

Drury, D.G. 1970. A fresh approach to the classification of the genus Gnaphalium with special reference to the species present in New Zealand (Inuleae-Compositae). New Zealand Journal of Botany 8: 222-248.

Endress, P. 2010. Disentangling confusions in inflorescence morphology: Patterns and diversity of reproductive shoot ramification in angiosperms. Journal of Systematics and Evolution 48 (4): 225-239.

FreIre, S.E. 1998. Tribu Inuleae (Compositae). In: R. Spichiger \& L. Ramella (eds.), Flora del Paraguay 27. Editions des Conservatoire et Jardin botaniques de la Ville de Genèv \& Missouri Botanical Garden, pp. 9-100.

Freire, S.E. \& L. Iharlegui. 2008. Asteraceae. Gnaphalium. In: F.O. Zuloaga, O. Morrone \& M.J. Belgrano (eds.), Catálogo de las Plantas Vasculares del Cono Sur de América del Sur: Argentina, Sur de Brasil (Paraná, Santa Catarina y Rio Grande do Sul), Chile, Paraguay y Uruguay 2. Monographs in Systematic Botany from the Missouri Botanical Garden, pp. 1312-1320.

Freire, S.E., L.P. Deble \& L. Iharlegui. 2011. Compostas Tribo Inuleae. In: A. Reis (ed.), Flora Ilustrada Catarinense; Parte 1. Herbário Barboza Rodriguez, Santa Catarina, pp.1067-1197.

Freire, S.E., C. Monti, A. Moreira Muñoz \& N.D. Bayón. 2013. Pseudognaphalium munoziae (Gnaphalieae, Asteraceae): A new South American species from Chile. Phytotaxa 105 (1): $1-10$.

Groves, B.E. 1977. Contributions to a chromosome atlas of the
New Zealand flora 19. Gnaphalium (Compositae). New Zealand Journal of Botany 15: 17-18.

Harris, J.G. \& M.W. Harris. 1994. Plant Identification Terminology: An Illustrated Glossary. Spring Lake Publishing, Spring Lake, Utah, 197 pp.

Hilliard, O.M. 1983. Flora of Southern Africa, Part 7 Inuleae, Fasc. 2 Gnaphaliinae. Government Printer, Pretoria, South Africa. Balogh Scientific Books (ed.). 325 pp.

Hilliard, O.M. \& B.L. Burtt. 1981. Some generic concepts in Compositae-Gnaphaliinae. Botanical Journal of the Linnean Society 82: 181-232.

Hind, D.J.N. 2011. An annotated preliminary checklist of the Compositae of Bolivia. Version 2. [See www.kew.org/ science/tropamerica/boliviacompositae for the web version and www.kew.org/science/tropamerica/boliviacompositae/ checklist.pdf for the pdf file of the checklist] c. 750 pp. 18.03.2013.

IBODA. 2014. Instituto de Botánica Darwinion. Disponible: $<$ http://www2.darwin.edu.ar $>$ [Consulta: 16-IV-2014].

KeIL, D.J. 1981. IOPB. Chromosome number reports 72. Taxon 30: 705-706.

KeIL, D.J. \& T.F. Stuessy. 1977. Chromosome counts of Compositae from Mexico and United States. American Journal of Botany 64: 791-798.

KLATt, F. W. 1878. Die Gnaphalien Amerikas. Eine Studie. Linnaea 42: 111-144.

Marticorena, C. \& M. Quezada. 1985. Catálogo de la Flora Vascular de Chile. Gayana, Botánica 42: 1-157.

McNeill, J., F.R. Barrie, W.R. Buck, V. Demoulin, W. Greuter, D.L. Hawksworth, P.S. Herendeen, S. Knapp, K. Marhold, J. Prado, W.F. Prud`homme van Reine, G.F. Smith, J.H. Wiersema \& N.J. Turland (eds.). 2012. International Code of Nomenclature for algae, fungi, and plants (Melbourne Code), adopted by the Eighteenth International Botanical Congress Melbourne, Australia, July 2011. Regnum Vegetabile 154. Koeltz Scientific Books.

Mehra, P.N. \& P. Remanandan. 1975. Cytological investigations on Indian Compositae 4. Tribes Senecioneae, Eupatorieae, Vernonieae, and Inuleae. The Nucleus 18: 6-19.

Monti, C., N.D. Bayón, D.A. Giuliano \& S.E. Freire. 2013. New Combinations and New Synonyms in Pseudognaphalium (Asteraceae: Gnaphalieae) from South America. Journal of the Botanical Research Institute of Texas 7(1): 195-202.

Muñoz Pizarro, C. 1960. Las especies de plantas descritas por R.A. Philippi en el siglo XIX. Ediciones Universidad de Chile, Santiago de Chile, $189 \mathrm{pp}$.

Nesom, G.L. 2001. New records in Pseudognaphalium (Gnaphalieae: Asteraceae) for the United States. Sida 19: 1185-1190.

Nesom, G.L. 2004. Pseudognaphalium canescens (Asteraceae: Gnaphalieae) and putative relatives in western North America. Sida 21: 781-790.

Nesom, G.L. 2006. Pseudognaphalium: 415. In: Flora of North America Editorial. Committee (eds.), Flora of North America North of Mexico. Oxford University Press, New York and Oxford 19.

NIE, Z.L, V.FunK, H. Sun, T. Deng, Y. Meng \& J. Wen. 2013. Molecular phylogeny of Anaphalis (Asteraceae, Gnaphalieae) with biogeographic implications in the Northern Hemisphere. Journal of Plant Research 126: 17-32. 
PhILIPPI, R.A. 1895. Gnaphalium. Pp: 7-26. Plantas nuevas chilenas de las familias que corresponden al tomo IV de la obra de Gay (continuación). Anales de la Universidad de Chile 90: 5-44.

Ramayya, N. 1962. Studies on the trichomes of some Compositae I. General structure. Bulletin of the Botanical Survey of India 4(1-4): 177-182.

Reiche, C. 1905. Gnaphalium. Pp: 47-65. Estudios críticos sobre la Flora de Chile 4, Santiago de Chile, 217 pp.

RÉmy, E.J. 1849. Compuestas. En: C. Gay (ed.), Historia física y política de Chile según documentos adquiridos en esta República durante doce años de residencia en ella. Botánica, Tomo 4, pp: 5-317. Paris.

RuA, G.H. 1999. Inflorescencias. Bases Teóricas para su Análisis. Sociedad Argentina de Botánica, Buenos Aires, 100 pp.

Smissen, R.D., M. Galbany-Casals \& I. Breitwieser. 2011. Ancient allopolyploidy in the everlasting daisies (Asteraceae: Gnaphalieae): complex relationships among extant clades. Taxon 60: 649-662.

StAfleu, F.A. \& R.S. Cowan. 1979. Taxonomic Literature, Vol. 2:

INDEX OF SCIENTIFIC NAMES

Synonyms are in italics, accepted names in bold face.

Achyrocline 72

Anaphalis DC. 69

Chevreulia pusilla DC. 103

Gamochaeta Wedd. 69, 72

Gamochaeta oligantha (Phil.) L.E.Navas 103

Gnaphalium L. p.p. excl. type 69, 72

subg. Laphangium (Hilliard \& B.L. Burtt) P.D. Sell 72

sect. Calolepis Kirp. 72

acutifolium Phil. 76

aldunateoides J. Rémy 73, 75

andicola Phil. 98, 100

araucanum Phil. 76, 79

argyrolepis Phil. 87, 89

cabrerae S.E. Freire 76

canum Phil. 81, 83

cheiranthifolium Lam. 76, 79, 100

var. multiflorum J. Koster 79

var. paniculatum (Bertero ex Colla) Skottsb. 79

chilense Spreng. 103

citrinum Hook. \& Arn. 76

coquimbense Phil. 98, 100

cymatoides Kunze ex DC. 81

var. glabrum Walp. 81

diminutivum Phil. 75

dysodes Spreng. 94

fastigiatum Phil. 76

gayanum J. Rémy 83, 85

glandulosum Klatt 91, 94

heterophyllum Phil. 85

heterotrichum Phil. 72, 85

illapelinum Phil. 98, 100

insulare Phil. 73, 75

lacteum Meyen \& Walp. 87, 89

landbeckii Phil. 89

leucocephalum Phil. 85
H-Le. 2nd ed. Regnum Veg. 98.

Thiers, B. 2011. Index Herbariorum: A global directory of public herbaria and associated staf. New York Botanical Garden's Virtual Herbarium. htpp://sweetgum.nybg.org/ ih/: 19.03.2013.

Troll, W. 1964-1969. Die Infloreszenzen. Typologie und Stellung im Aufbau des Vegetationskörpers, Bd. I (1964), II/I (1969). Jena.

Turner, B.L. 1970. Chromosome numbers in Compositae 12. Australian species. American Journal of Botany 57: 382389.

Ward, J., R.J. Bayer, I. Breitwieser, R. Smissen, M. GalbanyCasals \& M. Unwin. 2009. Gnaphalieae. In: V.A. Funk, A. Susanna, T.F. Stuessy \& R.J. Bayer (eds.), Systematics, Evolution, and Biogeography of Compositae, pp. 539588. Vienna, Austria: International Association for Plant Taxonomy (IAPT).

Weberling, F. 1985. Aspectos modernos de la morfología de las inflorescencias. Boletín de la Sociedad Argentina de Botánica 24(1-2): 1-28.

longifolium Phil. 98, 100

luteoalbum L. 73

moelleri Phil. 81, 83

montevidense Spreng. 98, 100

oliganthum Phil. 103

oxyphyllum DC. 69,72

paniculatum Colla 79

pedunculatum (Poepp. \& Endl.) Benth. \& Hook. ex Klatt 103

perpusillum Phil. 73, 75

phaeolepis Phil. 75

philippii Cabrera 76

pratense Phil. 98, 100

pseudohelichrysum Reiche 85

psilophyllum Meyen \& Walp. 91, 94

puberulum DC. 103

ramosum Phil. 103

remyanum Phil. 94

rivulare Phil. 75

robustum Phil. 85

sprengelii Hook. \& Arn. 103

subnudum Phil. 98, 100

suffruticosum Phil. 103

tarapacanum Phil. 96

ulophyllum Hook. \& Arn. 81

valdivianum Phil. 79

viravira Molina 98, 100

Helichrysum Mill. 69, 72

Hypelichrysum Kirp. 72 heterotrichum (Phil.) Kirp. 72

Laphangium (Hilliard \& B.L. Burtt) Tzvelev 69, 72

Lucilia pedunculata Poepp. \& Endl. 103

Pseudognaphalium Kirp. 69, 72

subgen. Laphangium Hilliard \& B.L. Burtt 72

aldunateoides (J. Rémy) C. Monti N. Bayón \& S.E. Freire $69,70,71,72,73,74,75$ 
andicola (Phil.) C. Monti N. Bayón \& S.E. Freire 98 cabrerae (S.E. Freire) Deble 69, 70, 71, 73, 76, 77, 79 cheiranthifolium (Lam.) Hilliard \& B.L. Burtt 69, 70, 71, $72,73,76,78,79,100$ coquimbense (Phil.) Anderb. 98 cymatoides (Kunze ex DC.) Anderb. 69, 70, 71, 73, 81, 82, 83,85

fastigiatum N. Bayón 76

gayanum (J. Rémy) Anderb. 69, 70, 71, 73, 81, 83, 84, 85 glandulosum (Klatt) Anderb. 91 heterotrichum (Phil.) Anderb. 72, 85

illapelinum (Phil.) Anderb. 98

lacteum (Meyen \& Walp.) Anderb. 69, 70, 71, 73, 87, 88, 89

landbeckii (Phil.) Anderb. 69, 70, 71, 73, 89, 90

luteoalbum (L.) Hilliard \& B.L. Burtt. 73, 98, 100 moelleri (Phil.) Anderb. 81

montevidense (Spreng.) Anderb. 98

munoziae N. Bayón C. Monti \& S.E. Freire 70, 71, 72, 73,

91, 92

oxyphyllum (DC.) Kirp. 72

perpusillum (Phil.) C. Monti N. Bayón \& S.E. Freire 73

pratense (Phil.) Anderb. 98

psilophyllum (Meyen \& Walp.) Anderb. 70, 71, 73, 91, 93, 96

puberulum DC. 102

remyanum (Phil.) Anderb. 69, 70, 71, 73, 94, 95, 96

robustum (Phil) Anderb. 85

tarapacanum (Phil.) Anderb. 70, 71, 73, 96, 97

viravira (Molina) Anderb. 69, 70, 71, 72, 73, 98, 99, 100, 103

INDEX TO NUMBERED COLLECTIONS EXAMINED

The number in parentheses refers to the corresponding species number in text.

A.J.H. 680 (12). Aedo 6806 (5); 7351 (3). Angulo 105 (3). Antoniz s.n. (6). Aravena 271 (3). Arellano 48720 (12). Arriaga s.n. (5). Arriagada s.n. (4). Arroyo 84-691 (6); 85-586 (9); 81112-A (11). Arroyo \& Humaña 99887 (4); 991714 (1). Arroyo et al. 85-393 (9); 97069 (9). Atanasio 706 (2).

Baeza \& López 2759 (3). Baeza et al. 3074 (12); 3110 (12). Barnier 225 (3); 427 (12); 488 (7). Barrientos 1621 (4); 1699 (4); 2025 (4). Barros 12 (12); 18 (12); 34 (1); 40 (5); 44 (12); 45 (1); 184 (12); 195 (5); 477 (12); 649 (1); 1466 (12); 1467 (5); 1940 (2); 2255 (4); 2258 (1); 2260 (4); 2272 (1); 2279 (3); 2285 (12); 2290 (1); 2301 (12); 2303 (9); 2304 (12); 2307 (4); 2318 (5); 2321 (5); 2417 (12); 2424 (12); 2840 (12); 3139 (12); 3370 (12); 3404 (5); 3874 (5); 6076 (12); 7332 (9); 21755 (12); 22611 (12); 24725 (5). Bayer 42 (5). Behn in 1941 (3); in 1928, in 1945 (4); in 1948 (5); in 1943. (12); 20879 (12); 24413 (5). Belisario 849 (12). Belmonte 20060 (9); 20154 (5). Bertero 1823 (12, type of Gnaphalium viravira). Bliss 674 (4); 2272 (1). Bliss \& Lusk 575 (4). Böcher et al. 543 (5). Boelcke 363 (12); 2424 (1); 3846 (5); 3885 (5). Buchtien s.n. (5); 3 (5), 174 (10). Bultmann 23661 (5). Burkart 9383 (4); 9491 (12).

Cabrera 3506 (5); 3636 (12); 11415 (5); 11462 (7); 12215 (12); 12523 (12); 12528 (5); 12697 (5); 19677 (12). Calderón s.n. (5). Cañulaf s.n. (4). Chang 20965 (12). Collantes s.n. (5). Crisci 349 (3); 490 (5). Cuming 334 (4); s.n. (1).

De Barba 2040 (12). De Vore 1545 (5). Dessauer s.n. (5). Díaz s.n. (1, type of Gnaphalium diminutivum).

Elgueta 546 (2). Escudero s.n. (5).

Fernández 1494 (5). Finckh 552 (12). Finot \& Leppe 97 (3); Finot \& López 1804 (12); s.n. (12). Frumau 63 (1, type of Gnaphalium phaeolepis). Fuhrmann s.n. (3).

Garaventa 320 (5); 1151 (9, type of Gnaphalium psilophyllum); 1965 (3); 3117 (5); 5681 (12); 1293 (5). García 4083 (5). Gardner et al. 295 (12). Gay s.n./s.f. (4); 678 (12, type of Gnaphalium coquimbense); 681 (5, type of Gnaphalium heterotrichum); 739 (1, type of Gnaphalium perpusillum). Geisze s.n. (5, type of Gnaphalium heterophyllum). Germain s.n. (1, type of Gnaphalium insulare). Gleisner s.n. (12); 201 (12). Grandjot s.n. (12); 995 (5). C. \& G. Grandjot s.n. (12). Gunckel in 1924, in 1933 (3); in 1943 (4); in 1955 (5); 374 (12); 819 (12); 1488 (3); 2999 (3); 3196 (3); 3324 (3); 11011 (4); 12826 (3); 12827 (4); 19193 (3); 21612 (4); 22280 (12); 22760 (12); 23443 (3); 23453 (12); 28012 (3); 30004 (5); 32025 (5); 36408 (3); 39466 (12); 40170 (4); 40215 (3); 40589 (3); 40598 (12); 41862 (3); 43572 (3); 46477 (7); 46993 (1); 48696 (9).

Harvey s.n. (4). Hastings 418 (12). Hicken 122 (5). Hochleitner s.n. (3). Hollermayer s.n. (4). Humaña et al. 20102 (12). Hutchinson 67 (5).

Ibáñez et al. s.n. (5). Igaymán \& M. Muñoz s.n. (5).

Jaffuel s.n. (4); 896 (12); 899 (12); 1306 (3); 2681 (5); 3267 (5). Jaffuel \& Pirion 3026 (4); 3226 (4). Jiles s.n. (5); 542-a (5); 910 (12); 1112 (4); 1686 (4); 1818 (12); 1848 (5); 3047 (5); 3429 (5); 3887 (9); 4428 (12); 4735 (12); 6083 (6); 6437 (5). Joseph 3657 (5). Junge in 1934 (3); in 1940 (4); in 1936 (12); 6285 (12); 6767 (1).

Kausel 3806 (5). Kuntze s.n. (12).

Lammers et al. 6381 (5). Landbeck in 1860 (5, type of Gnaphalium leucocephalum); XII-1861 (7, type of Gnaphalium landbeckii); XII-1862 (12, type of Gnaphalium illapelinum). Landero et al. 48 (6). Landrum \& Landrum 8887 (9). Ledezma 8 (4); 225 (11). Levi Heins 150 (4); 154 (3); 174 (4); 175 (5); 306 (11); 445 (5); 2911 (3). Looser 203 (12); 3765 (5); 3990 (12); 4394 (5); 66209 (3); 66226 (4); 66253 (5). López 11403 (3). Luebert \& Teillier 2232 (12).

Mahu s.n. (12); 4885 (12); 8657 (5); 9795 (12); 10213 (12); 10335 (3). A. Marticorena \& Jiménez s.n. (5); 214 (3). C. Marticorena 
1591 (12). C. Marticorena \& Furet 62 (3). C. Marticorena \& Matthei 49 (9); 67 (9); 967 (12); 973 (12). C. Marticorena \& Rodríguez 8417 (12); 8430 (12). C. Marticorena et al. s.n. (12); 14 (6); 21 (9); 29 (11); 43 (12); 46 (5); 141 (9); 142 (6); 227 (6); 244 (6); 276 (4); $316(6) ; 353$ (3); 360 (11); 421 (12); 468 (5); 779 (12); 1006 (3); 1307 (3); 1338 (4); 1351 (12); 1612 (5); 9956 (5); 9972 (4); 83413 (12). C. Martínez s.n. (5). G. Martínez 49072 (5). Matte 745 (12). Matthei \& Quezada 463 (5); 1420 (1). Matthei \& Rodríguez 269 (4); 299 (3). Meyer 9684 (3). Mihoc 4 (4); 60 (4). Mihoc \& Teneb 3897 (3). Mihoc et al. 3308 (3); 6227 (1). Montero 222 (3); 1881 (5); 3667 (12); 5728 (1); 6056 (12); 6364 (4); 6396 (12); 9005 (3); 9437 (5); 10151 (3); 11575 (3); 12045 (2); 12281 (12). Moreira 1953 (8). Moreira et al. 1676 (8, type of Pseudognaphalium munoziae). Moreno s.n. (5); 25689 (12). H. Muñoz 48718 (12); Muñoz \& Sierra 7327 (3).

Navarro s.n. (12, type of Gnaphalium andicola). Navas in 1953 (4); in 1954 (4); 632 (4); 678 (11); 1389 (5). Niemeyer \& Fernández 8908 (12). Niemeyer et al. 8970 (9); 89101 (6); 89106 (6); 89122 (5). Novoa s.n. (3); s.n. (12).

Oehrens s.n. (12). Ojeda 20949 (7).

Palma \& Inostroza s.n. (3); Parra 37 (1); 475 (5). Parra \& Torres 163 (3); 223 (3); 431 (12); 782 (3); 819 (3); 1026 (3). Pennell 12845 (4). Pfanzelt 173 (12). Pfister in 1935, in 1946. (3) in 1951, in 1955, in 1956 (4); in 1948, in 1952 (5); in 1950 (9); in I-1950 (11); in 1944, in 1947, in 1948, in 1949 (12); 786 (3); 4277 (12); 4278 (3); 10502 (1). Pfister \& Ricardi s.n. (3); s.n. (12). Philippi 18381842 (4); I-1860 (12, type of Gnaphalium pratense); in 1877 (10, type of Gnaphalium remyanum); III-1878 (2, type of Gnaphalium fastigiatum); II-1882 (12, type of Gnaphalium longifolium); I-1884 (12); II-1885 (11, type of Gnaphalium tarapacanum); III-1885 (4, type of Gnaphalium moelleri); 17-II-1885 (6); III-1887 (4, type of Gnaphalium canum); I-1888 (5, type of Gnaphalium robustum); I-1893 (3, type of Gnaphalium acutifolium); s.n./s.f. (5). Pisano et al. 1567 (12).

Rabanales s.n. (12). Rahmer III-1885 (6, type of Gnaphalium argyrolepis); I-1886 (11, type of Gnaphalium tarapacanum) Ricardi 20-XII-1950 (3); 29-XII-1950 (12); 2448 (12); 2853 (5); 3157 (12); 5445 (1); 9259 (1). Ricardi \& C. Marticorena 3747 (12); 3956 (5); 4222 (12); 4223 (5); 4488 (5); 4730 (9); 4807 (6);
4943 (4); 4943 bis (4); 4972 (5); 5052 (12); 5150 (12); 5721 (12); 5766 (12). Ricardi \& Silva 3535 (9); 3544 (6). Ricardi et al. 155 (5); 279 (11); 286 (9); 325 (6); 398 (9); 788 (6); 790 (5); 796 (5); 834 (12); 1348 (12); 1551 (12). Richter s.n. (12). R. Rodríguez 661 (12); 1476 (3); 2789 (5). R. Rodríguez \& Baeza 2371 (4). Rodríguez \& C. Marticorena 3053 (12). R. Rodríguez \& Pacheco 1912 (12). Roesner s.n. (5); 6508 (1). Rosas 1385 (5); 1392 (5); 1465 (5); 1507 (5); 4799 (5); 4946 (11). E. Ruiz \& P. López 1018 (12); 1184 (12); 1231 (11).

Saa s.n. (5). Saavedra \& Pauchard 111 (12). Santos 154 (3). Schlegel 433 (5); 1537 (3); 3125 (12); 3144 (12); 3199 (4); 3329 (5); 4895 (4); 4949 (12); 5863 (12); 8034 (5). Semler s.n. (9). J. \& A. Solomon 4226 (3). Sparre 121 (3); 11016 (5). Sparre \& Smith 80 (3). Stuessy \& Crawford 6436 (3). Stuessy \& López 11449 (3); Stuessy et al. 6201 (3). Swenson \& Stuessy 399 (3). S/leg. (4).

Teillier 3224 (1); 4624 (5); 5111 (5); 5851 (5); 5915 (9); 6489 (5); 51112 (1). Teillier \& González 2301 (12). Teillier \& Márquez 4848 (5); 4870 (4); 5313 (5); 5314 (5); 5315 (12); 5316 (1). Teillier \& Niemeyer 3322 (4). Teillier et al. 2027 (5). Teneb 551 (12); 569 (12). Tepe 2003 (12). Tepe et al. 1688 (5); 1689 (12); 1727 (3); 1840 (12); 1923 (3). Tomé 128 (5); 129 (5); 130 (12).

Ugarte 174 (3). Urtubey et al. 700 (1).

Vera \& Ortiz 21 (3); 136 (3); 151 (3). Villagrán 5699 (3). Villagrán \& Armesto 2271 (9). Villagrán \& Leiva 7269 (3); 7448 (9); 7545 (3). Villagrán et al. 1271 (11); 2353 (9); 2441 (9); 2478 (9); 9098 (9); 9229 (8); 9237 (9). Villarroel \& Weldt 123 (3). Volkmann s.n. (3, type of Gnaphalium araucanum). Von Bayern 388 (5). Von Bohlen 742 (5). Von Rentzell 6138 (12).

Wagenknecht s.n. (3); 4396 (5). Weisser Sievers s.n. (5). Weldt \& Rodríguez 946 (3); 1149 (11). Werdermann 37 (3); 107 (5); 470 (5); 1092 (6); 1100 (9). Wilkes s.n. (12). Worth \& Morrison 16494 (5).

Zemelman s.n. (4). Zollitsch 22 (5). Zöllner 976 (9); 978 (5); 1068 (4); 1069 (5); 1076 (5); 1138 (5); 1770 (12); 1772 (5); 2200 (5); 3031 (12); 5133 (12); 7936 (12); 8088 (5); 9914 (12); 10151 (5); 10198 (5); 10442 (5); 11254 (9); 11790 (5); 11991 (9); 12142 (12); 12518 (5); 13111 (12); 13147 (12); 13652 (12).

Recibido: 31.07 .13

Aceptado: 28.04.14 